\title{
N-Heterocyclic Carbene-Catalyzed Radical Relay Enabling Vicinal Alkylacylation of Alkenes
}

Takuya Ishii, Kenji Ota, Kazunori Nagao*, and Hirohisa Ohmiya*

Division of Pharmaceutical Sciences, Graduate School of Medical Sciences, Kanazawa University, Kakuma-machi, Kanazawa 920-1192, Japan

*E-mail: Kazunori Nagao : nkazunori@p.kanazawa-u.ac.jp

Hirohisa Ohmiya: ohmiya@p.kanazawa-u.ac.jp

\section{Table of Contents}

- Instrumentation and Chemicals

S2

- Procedure for Alkylacylation of Alkenes

S3

- Characterization Data for product ketones

S3-S17

- Effect of NHC Catalyst on Two- and Three-Component Coupling

$\mathrm{S} 18$

- References

S19

- NMR Spectra 


\section{- Instrumentation and Chemicalsa}

NMR spectra were recorded on a JNM-ECS400, operating at $400 \mathrm{MHz}$ for ${ }^{1} \mathrm{H}$ NMR and 100.5 $\mathrm{MHz}$ for ${ }^{13} \mathrm{C} \mathrm{NMR}$, and JNM-ECA600, operating at $600 \mathrm{MHz}$ for ${ }^{1} \mathrm{H} \mathrm{NMR}$ and $150.9 \mathrm{MHz}$ for ${ }^{13} \mathrm{C}$ NMR. Chemical shift values for ${ }^{1} \mathrm{H}$ and ${ }^{13} \mathrm{C}$ are referenced to $\mathrm{Me}_{4} \mathrm{Si}$ and the residual solvent resonances, respectively. Chemical shifts are reported in $\delta \mathrm{ppm}$. Mass spectra were obtained with JMS-T100TD (DART). TLC analyses were performed on commercial glass plates bearing 0.25-mm layer of Merck Silica gel 60F 254 . Silica gel (Kanto Chemical Co., Silica gel 60 N, spherical, neutral) was used for column chromatography. IR spectra were measured with a Thermo Scientific iD7 ATR Accessory for the Thermo Scientific Nicolet iS5 FT-IR Spectrometer. Melting points were measured on a Yanaco MP-500D apparatus.

All reactions were carried out under nitrogen or argon atmosphere. Materials were obtained from commercial suppliers or prepared according to standard procedures unless otherwise noted. $\mathrm{K}_{2} \mathrm{CO}_{3}$, DBU and TMG were purchased from Tokyo Chemical Industry Co., stored under nitrogen, and used as received. $\mathrm{Cs}_{2} \mathrm{CO}_{3}$ was purchased from FUJIFILM Wako Pure Chemical Co., stored under nitrogen, and used as received. DMSO was purchased from FUJIFILM Wako Pure Chemical Co., stored under nitrogen, and used as received. Aldehydes $\mathbf{1 a}, \mathbf{1 e}, \mathbf{1 h}, \mathbf{1} \mathbf{i}$ and $\mathbf{1} \mathbf{k}$ were purchased from Nacalai Tesque Inc., 1b, 1g and 1n were purchased from FUJIFILM Wako Pure Chemical Co., 1c, 1d, 1f, 1j, $1 \mathbf{l}$ and 1m were purchased from Tokyo Chemical Industry Co., stored under nitrogen, and used as received. Alkenes $\mathbf{2 b}-\mathbf{2 h}$ were prepared by Wittig reaction from the corresponding aldehydes. Alkenes $\mathbf{2 a}$ and $\mathbf{2 i}$ were purchased from FUJIFILM Wako Pure Chemical Co., $\mathbf{2} \mathbf{j}$ was purchased from Sigma-Aldrich Japan, distilled before use. The redox-active esters 3 were prepared by the reported procedure. ${ }^{1}$ Thiazolium salt N1-N5 were prepared by the reported procedure. ${ }^{2}$ 


\section{- Procedure for Alkylacylation of Alkenes}

The reaction in Table 1, entry 1 is representative. Thiazolium salt $\mathbf{N 1}(4.0 \mathrm{mg}, 0.01 \mathrm{mmol})$ and redox-active esters 3a $(116.5 \mathrm{mg}, 0.3 \mathrm{mmol})$ were placed in a schlenk tube containing a magnetic stirring bar. The vial was sealed with a Teflon ${ }^{\circledR}$-coated silicon rubber septum, and then the vial was evacuated and filled with argon. Degassed DMSO $(400 \mu \mathrm{L})$ was added to the vial. Next, benzaldehyde (1a) $(20.8 \mu \mathrm{L}, 0.2 \mathrm{mmol})$ and styrene (2a) $(45.8 \mu \mathrm{L}, 0.4 \mathrm{mmol})$ were added. Then, $\mathrm{Cs}_{2} \mathrm{CO}_{3}(6.5 \mathrm{mg}$, $0.02 \mathrm{mmol}$ ) was added. After $4 \mathrm{~h}$ stirring at $80{ }^{\circ} \mathrm{C}$, the reaction mixture was treated with saturated $\mathrm{NH}_{4} \mathrm{Cl}$ aqueous solution $(400 \mu \mathrm{L})$, then extracted with diethylether (4 times) and dried over sodium sulfate. After filtration, the resulting solution was evaporated under reduced pressure. After volatiles were removed under reduced pressure, flash column chromatography on silica gel (100:0-94:6, hexane/EtOAc) gave 4aaa (68.5 mg, $0.17 \mathrm{mmol})$ in $84 \%$ yield.

\section{- Characterization Data for Aryl Alkyl Ketones}

Due to difficulties of purification, ketones 4 aao and 4aea were isolated as the corresponding alcohols 4aao' and 4aea' respectively by treating the crude ketones with $\mathrm{NaBH}_{4}$ followed by column chromatography.

tert-Butyl 4-Benzoyl-4-methylpiperidine-1-carboxylate (5aa)

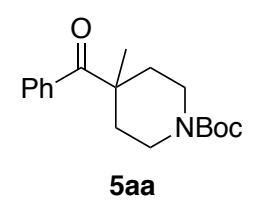

Brown oil. IR (neat) 1106, 1151, 1248, 1365, 1419, 1597, $1686 \mathrm{~cm}^{-1} .{ }^{1} \mathbf{H}$ NMR $\left(600 \mathrm{MHz}, \mathrm{CDCl}_{3}\right)$ $\delta 1.44(\mathrm{~s}, 9 \mathrm{H}), 1.45(\mathrm{~s}, 3 \mathrm{H}), 1.54-1.56(\mathrm{~m}, 2 \mathrm{H}), 2.24-2.27(\mathrm{~m}, 2 \mathrm{H}), 3.05-3.08(\mathrm{~m}, 2 \mathrm{H}), 3.63(\mathrm{brs}, 2 \mathrm{H})$, $7.41(\mathrm{t}, J=7.2 \mathrm{~Hz}, 2 \mathrm{H}), 7.48(\mathrm{t}, J=7.2 \mathrm{~Hz}, 1 \mathrm{H}), 7.68(\mathrm{~d}, J=7.2 \mathrm{~Hz}, 2 \mathrm{H}) .{ }^{13} \mathbf{C}$ NMR $(150.9 \mathrm{MHz}$, $\left.\mathrm{CDCl}_{3}\right) \delta 25.3,28.4,35.2,40.8,37.9,46.6,79.4,127.8,128.3,131.2,138.5,154.8,207.8$. HRMSDART $(\mathrm{m} / \mathrm{z})$ : $[\mathrm{M}]^{+}$calcd for $\mathrm{C}_{18} \mathrm{H}_{25} \mathrm{NO}_{3}, 303.1829$; found, 303.1842 .

\section{tert-Butyl 4-Methyl-4-(3-oxo-2,3-diphenylpropyl)piperidine-1-carboxylate (4aaa)}

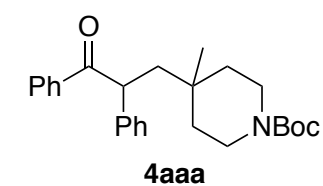

The product 4aaa was purified by flash chromatography on silica gel (100:0-94:6, hexane/EtOAc) (Table 1, entry 1; $68.5 \mathrm{mg}, 0.17 \mathrm{mmol}, 84 \%$ isolated yield). White solid. M.p. $122-124{ }^{\circ} \mathrm{C}$. IR (neat) 1089, 1156, 1222, 1246, 1364, 1422, 1475, 1597, $1673 \mathrm{~cm}^{-1} .{ }^{1} \mathbf{H} \mathbf{N M R}\left(600 \mathrm{MHz}, \mathrm{CDCl}_{3}\right) \delta 0.93(\mathrm{~s}$, $3 \mathrm{H}), 1.20-1.22(\mathrm{~m}, 2 \mathrm{H}), 1.26-1.37(\mathrm{~m}, 2 \mathrm{H}), 1.43(\mathrm{~s}, 9 \mathrm{H}), 1.64(\mathrm{dd}, J=14.4,3.0 \mathrm{~Hz}, 1 \mathrm{H}), 2.68(\mathrm{dd}, J$ $=14.4,8.4 \mathrm{~Hz}, 1 \mathrm{H}), 3.12-3.13(\mathrm{~m}, 2 \mathrm{H}), 3.52-3.56(\mathrm{~m}, 2 \mathrm{H}), 4.74(\mathrm{dd}, J=8.4,3.0 \mathrm{~Hz}, 1 \mathrm{H}), 7.18(\mathrm{~m}$, $1 \mathrm{H}), 7.26-7.31(\mathrm{~m}, 4 \mathrm{H}), 7.41(\mathrm{t}, J=7.2 \mathrm{~Hz}, 2 \mathrm{H}), 7.50(\mathrm{t}, J=7.2 \mathrm{~Hz}, 1 \mathrm{H}), 7.98(\mathrm{~d}, J=7.2 \mathrm{~Hz}, 2 \mathrm{H})$. ${ }^{13} \mathbf{C}$ NMR $\left(150.9 \mathrm{MHz}, \mathrm{CDCl}_{3}\right) \delta 23.7,28.4,32.2,37.0,39.7,45.6,48.5,79.2,126.9,128.0,128.58$, 128.61, 129.0, 132.9, 136.7, 140.8, 154.9, 199.5. HRMS-DART $(\mathrm{m} / z)$ : $[\mathrm{M}]^{+}$calcd for $\mathrm{C}_{26} \mathrm{H}_{33} \mathrm{NO}_{3}$, 407.2455; found, 407.2459 . 


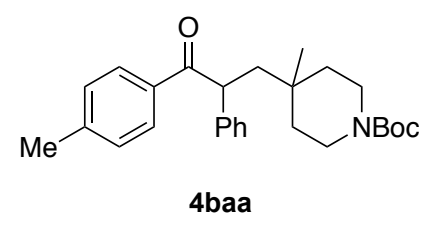

The product 4baa was purified by flash chromatography on silica gel (98:2-92:8, hexane/EtOAc) (Table 2; $71.7 \mathrm{mg}, 0.17 \mathrm{mmol}, 85 \%$ isolated yield). White solid. M.p. $107-110{ }^{\circ} \mathrm{C}$. IR (neat) 1161, 1246, 1278, 1364, 1423, 1605, $1678 \mathrm{~cm}^{-1} .{ }^{1} \mathbf{H}$ NMR (600 MHz, $\left.\mathrm{CDCl}_{3}\right) \delta 0.93(\mathrm{~s}, 3 \mathrm{H}), 1.20-1.22(\mathrm{~m}$, $2 \mathrm{H}), 1.26-1.37(\mathrm{~m}, 2 \mathrm{H}), 1.42(\mathrm{~s}, 9 \mathrm{H}), 1.63(\mathrm{dd}, J=14.4,3.0 \mathrm{~Hz}, 1 \mathrm{H}), 2.36(\mathrm{~s}, 3 \mathrm{H}), 2.67$ (dd, $J=14.4$, $9.0 \mathrm{~Hz}, 1 \mathrm{H}), 3.10-3.15(\mathrm{~m}, 2 \mathrm{H}), 3.47-3.54(\mathrm{~m}, 2 \mathrm{H}), 4.72(\mathrm{dd}, J=9.0,3.0 \mathrm{~Hz}, 1 \mathrm{H}), 7.17$ (t, $J=7.2$ $\mathrm{Hz}, 1 \mathrm{H}), 7.21(\mathrm{~d}, J=8.4 \mathrm{~Hz}, 2 \mathrm{H}), 7.26(\mathrm{t}, J=7.2 \mathrm{~Hz}, 2 \mathrm{H}), 7.30(\mathrm{~d}, J=7.2 \mathrm{~Hz}, 2 \mathrm{H}), 7.89(\mathrm{~d}, J=8.4$ $\mathrm{Hz}, 2 \mathrm{H}) .{ }^{13} \mathbf{C}$ NMR $\left(150.9 \mathrm{MHz}, \mathrm{CDCl}_{3}\right) \delta 21.6,23.7,28.5,32.2,37.0,39.7,45.6,48.3,79.2,126.8$, 128.1, 128.8, 129.0, 129.3, 134.2, 141.1, 143.8, 154.9, 199.1. HRMS-DART $(\mathrm{m} / \mathrm{z}): \mathrm{M}^{+}$calcd for $\mathrm{C}_{27} \mathrm{H}_{35} \mathrm{NO}_{3}, 421.2611$; found, 421.2612.

tert-Butyl 4-[3-(4-Methoxyphenyl)-3-oxo-2-phenylpropyl]-4-methylpiperidine-1-carboxylate (4caa)

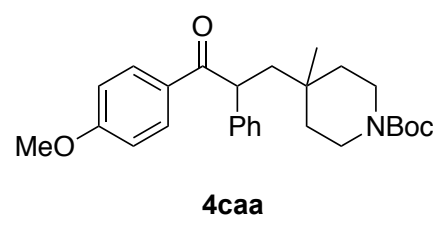

The product 4caa was purified by flash chromatography on silica gel (100:0-92:8, hexane/EtOAc) (Table 2; $78.8 \mathrm{mg}, 0.18 \mathrm{mmol}, 90 \%$ isolated yield). White solid. M.p. $100-103{ }^{\circ} \mathrm{C}$. IR (neat) 1165, 1246, 1262, 1364, 1420, 1599, $1676 \mathrm{~cm}^{-1} .{ }^{1} \mathbf{H}$ NMR (400 MHz, $\left.\mathrm{CDCl}_{3}\right) \delta 0.93(\mathrm{~s}, 3 \mathrm{H}), 1.18-1.21(\mathrm{~m}$, $2 \mathrm{H}), 1.27-1.35(\mathrm{~m}, 2 \mathrm{H}), 1.43(\mathrm{~s}, 9 \mathrm{H}), 1.62(\mathrm{dd}, J=14.0,3.2 \mathrm{~Hz}, 1 \mathrm{H}), 2.67(\mathrm{dd}, J=14.0,8.8 \mathrm{~Hz}, 1 \mathrm{H})$, $3.11-3.13(\mathrm{~m}, 2 \mathrm{H}), 3.51-3.55(\mathrm{~m}, 2 \mathrm{H}), 3.83(\mathrm{~s}, 3 \mathrm{H}), 4.70(\mathrm{dd}, J=8.8,3.2 \mathrm{~Hz}, 1 \mathrm{H}), 6.89$ (d, $J=8.8$ $\mathrm{Hz}, 2 \mathrm{H}), 7.17(\mathrm{~m}, 1 \mathrm{H}), 7.25-7.31(\mathrm{~m}, 4 \mathrm{H}), 7.98(\mathrm{~d}, J=8.8 \mathrm{~Hz}, 2 \mathrm{H}) .{ }^{13} \mathbf{C} \mathbf{N M R}\left(150.9 \mathrm{MHz}, \mathrm{CDCl}_{3}\right)$ $\delta 23.7,28.5,32.2,37.1,39.8,45.6,48.1,55.4,79.2,113.8,126.8,128.0,129.0,129.6,130.9,141.3$, 154.9, 163.4, 198.0. HRMS-DART $(\mathrm{m} / \mathrm{z})$ : $[\mathrm{M}]^{+}$calcd for $\mathrm{C}_{27} \mathrm{H}_{35} \mathrm{NO}_{4}, 437.2561$; found, 437.2564 .

tert-Butyl

4-Methyl-4-\{3-[4-(methylthio)phenyl]-3-oxo-2-phenylpropyl\}piperidine-1carboxylate (4daa)

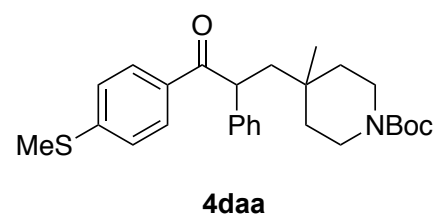

The product 4daa was purified by flash chromatography on silica gel (98:2-93:7, hexane/EtOAc) (Table 2; $78.9 \mathrm{mg}, 0.17 \mathrm{mmol}, 87 \%$ isolated yield). White solid. M.p. 104-106 ${ }^{\circ} \mathrm{C}$. IR (neat) 1093, 1160, 1246, 1278, 1364, 1424, 1587, $1675 \mathrm{~cm}^{-1} .{ }^{1} \mathbf{H}$ NMR (600 MHz, $\left.\mathrm{CDCl}_{3}\right) \delta 0.93$ (s, 3H), $1.19-$ $1.21(\mathrm{~m}, 2 \mathrm{H}), 1.26-1.32(\mathrm{~m}, 2 \mathrm{H}), 1.43(\mathrm{~s}, 9 \mathrm{H}), 1.63(\mathrm{dd}, J=14.4,3.6 \mathrm{~Hz}, 1 \mathrm{H}), 2.48(\mathrm{~s}, 3 \mathrm{H}), 2.67$ (dd, 
$J=14.4,8.4 \mathrm{~Hz}, 1 \mathrm{H}), 3.10-3.15(\mathrm{~m}, 2 \mathrm{H}), 3.52-3.54(\mathrm{~m}, 2 \mathrm{H}), 4.68(\mathrm{dd}, J=8.4,3.6 \mathrm{~Hz}, 1 \mathrm{H}), 7.18(\mathrm{~m}$, 1H), 7.21-7.22 (m, 2H), 7.25-7.29 (m, 4H), 7.90-7.91 (m, 2H). ${ }^{13} \mathbf{C}$ NMR (150.9 MHz, CDCl $\left.{ }_{3}\right)$ $\delta 14.7,23.7,28.4,32.2,37.0,39.7,45.5,48.2,79.2,125.0,126.9,128.0,129.0(\times 2 \mathrm{C}), 132.9,141.0$, 145.9, 154.9, 198.4. HRMS-DART $(m / z)$ : $[\mathrm{M}]^{+}$calcd for $\mathrm{C}_{27} \mathrm{H}_{35} \mathrm{NO}_{3} \mathrm{~S}, 453.2332$; found, 453.2336.

tert-Butyl 4-Methyl-4-\{3-oxo-2-phenyl-3-[4-(trifluoromethoxy)phenyl]propyl\}piperidine-1carboxylate (4eaa)

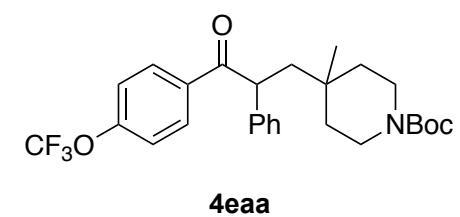

The product 4eaa was purified by flash chromatography on silica gel (98:2-93:7, hexane/EtOAc) (Table 2; $78.6 \mathrm{mg}, 0.16 \mathrm{mmol}, 80 \%$ isolated yield). White solid. M.p. $102-105{ }^{\circ} \mathrm{C}$. IR (neat) 1162 , $1211,1253,1365,1421,1601,1685 \mathrm{~cm}^{-1} .{ }^{1} \mathbf{H}$ NMR (600 MHz, $\left.\mathrm{CDCl}_{3}\right) \delta 0.93(\mathrm{~s}, 3 \mathrm{H}), 1.19-1.21(\mathrm{~m}$, $2 \mathrm{H}), 1.31-1.38(\mathrm{~m}, 2 \mathrm{H}), 1.43(\mathrm{~s}, 9 \mathrm{H}), 1.64(\mathrm{dd}, J=14.4,3.0 \mathrm{~Hz}, 1 \mathrm{H}), 2.67$ (dd, $J=14.4,8.4 \mathrm{~Hz}, 1 \mathrm{H})$, $3.11-3.14(\mathrm{~m}, 2 \mathrm{H}), 3.53-3.57(\mathrm{~m}, 2 \mathrm{H}), 4.67(\mathrm{dd}, J=8.4,3.0 \mathrm{~Hz}, 1 \mathrm{H}), 7.20(\mathrm{~m}, 1 \mathrm{H}), 7.23(\mathrm{~d}, J=8.4$ $\mathrm{Hz}, 2 \mathrm{H}), 7.26-7.30(\mathrm{~m}, 4 \mathrm{H}), 8.02(\mathrm{~d}, J=8.4 \mathrm{~Hz}, 2 \mathrm{H}) .{ }^{13} \mathbf{C} \mathbf{N M R}\left(150.9 \mathrm{MHz}, \mathrm{CDCl}_{3}\right) \delta 23.6,28.4$, $32.2,37.0,39.7,45.6,48.7,79.3,120.3$ (q, $J=260 \mathrm{~Hz}$ ), 120.4, 127.2, 128.0, 129.2, 130.6, 134.9, 140.4, 152.5, 154.9, 197.9. HRMS-DART $(\mathrm{m} / \mathrm{z})$ : $[\mathrm{M}]^{+}$calcd for $\mathrm{C}_{27} \mathrm{H}_{32} \mathrm{~F}_{3} \mathrm{NO}_{4}, 491.2278$; found, 491.2263.

tert-Butyl 4-Methyl-4-\{3-oxo-2-phenyl-3-[4-(trifluoromethyl)phenyl]propyl\}piperidine-1carboxylate (4faa)

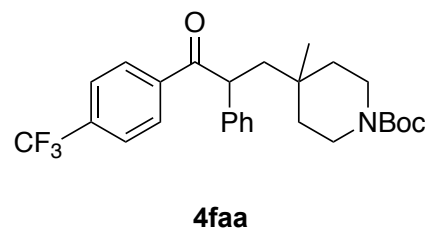

The product 4 faa was purified by flash chromatography on silica gel (100:0-95:5, hexane/EtOAc) (Table 2; 61.8. mg, $0.13 \mathrm{mmol}, 65 \%$ isolated yield). White solid. M.p. $112-115{ }^{\circ} \mathrm{C}$. IR (neat) 1066, 1130, 1163, 1247, 1323, 1409, 1582, $1686 \mathrm{~cm}^{-1} .{ }^{\mathbf{1}} \mathbf{H}$ NMR (600 MHz, $\left.\mathrm{CDCl}_{3}\right) \delta 0.93(\mathrm{~s}, 3 \mathrm{H}), 1.20$ $1.22(\mathrm{~m}, 2 \mathrm{H}), 1.26-1.32(\mathrm{~m}, 2 \mathrm{H}), 1.43(\mathrm{~s}, 9 \mathrm{H}), 1.66(\mathrm{dd}, J=14.4,3.6 \mathrm{~Hz}, 1 \mathrm{H}), 2.67(\mathrm{dd}, J=14.4,8.4$ Hz, 1H), 3.11-3.14 (m, 2H), 3.47-3.55 (m, 2H), 4.69 (dd, J=8.4, 3.6 Hz, 1H), 7.20 (m, 1H), 7.26$7.29(\mathrm{~m}, 4 \mathrm{H}), 7.67(\mathrm{~d}, J=8.4 \mathrm{~Hz}, 2 \mathrm{H}), 8.06(\mathrm{~d}, J=8.4 \mathrm{~Hz}, 2 \mathrm{H}) .{ }^{13} \mathbf{C} \mathbf{N M R}\left(150.9 \mathrm{MHz}, \mathrm{CDCl}_{3}\right)$ $\delta 23.6,28.4,32.2,37.0,39.7,45.5,49.1,79.3,125.3$ (q, $J=273 \mathrm{~Hz}), 125.7$ (q, $J=4.5 \mathrm{~Hz}), 127.3$, 128.0, 128.9, 129.3, 134.2 (q, $J=31.7 \mathrm{~Hz}), 139.5,140.1154 .9,198.5$. HRMS-DART $(\mathrm{m} / z):[\mathrm{M}]^{+}$ calcd for $\mathrm{C}_{27} \mathrm{H}_{32} \mathrm{~F}_{3} \mathrm{NO}_{3}, 475.2329$; found, 475.2318.

tert-Butyl

4-[3-(4-Fluorophenyl)-3-oxo-2-phenylpropyl]-4-methylpiperidine-1-carboxylate (4gaa) 


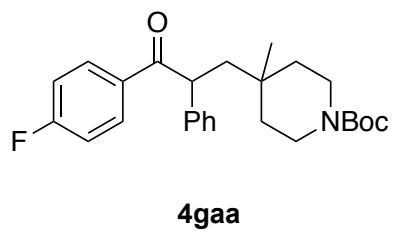

The product 4gaa was purified by flash chromatography on silica gel (98:2-94:6, hexane/EtOAc) (Table 2; $67.2 \mathrm{mg}, 0.16 \mathrm{mmol}, 79 \%$ isolated yield). White solid. M.p. $95-97^{\circ} \mathrm{C}$. IR (neat) 1156, 1232 , 1278, 1365, 1423, 1506, 1597, $1682 \mathrm{~cm}^{-1} .{ }^{1} \mathbf{H} \mathbf{~ N M R}\left(600 \mathrm{MHz}, \mathrm{CDCl}_{3}\right) \delta 0.93(\mathrm{~s}, 3 \mathrm{H}), 1.19-1.21(\mathrm{~m}$, $2 \mathrm{H}), 1.27-1.38(\mathrm{~m}, 2 \mathrm{H}), 1.43(\mathrm{~s}, 9 \mathrm{H}), 1.64(\mathrm{dd}, J=14.4,3.0 \mathrm{~Hz}, 1 \mathrm{H}), 2.66(\mathrm{dd}, J=14.4,8.4 \mathrm{~Hz}, 1 \mathrm{H})$, 3.12-3.14 (m, 2H), 3.50-3.54 (m, 2H), $4.67(\mathrm{dd}, J=8.4,3.0 \mathrm{~Hz}, 1 \mathrm{H}), 7.06-7.09(\mathrm{~m}, 2 \mathrm{H}), 7.19(\mathrm{~m}$, 1H), 7.26-7.30 (m, 4H), 7.99-8.02 (m, 2H). $\left.{ }^{13} \mathbf{C ~ N M R ~ ( 1 5 0 . 9 ~ M H z , ~} \mathrm{CDCl}_{3}\right) \delta 23.6,28.4,32.2,37.0$, 39.7, 45.5, 48.5, 79.2, 115.7 (d, $J=21.1 \mathrm{~Hz}), 127.0,128.0,129.1,131.2(\mathrm{~d}, J=9.1 \mathrm{~Hz}), 133.0(\mathrm{~d}, J$ $=3.0 \mathrm{~Hz}), 140.6,154.9,165.6(\mathrm{~d}, J=255 \mathrm{~Hz}), 197.9$. HRMS-DART $(\mathrm{m} / \mathrm{z}):[\mathrm{M}]^{+}$calcd for $\mathrm{C}_{26} \mathrm{H}_{32} \mathrm{FNO}_{3}$, 425.2361; found, 425.2382.

tert-Butyl 4-[3-(4-Chlorophenyl)-3-oxo-2-phenylpropyl]-4-methylpiperidine-1-carboxylate (4haa)

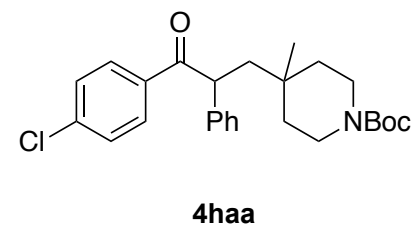

The product 4haa was purified by flash chromatography on silica gel (98:2-94:6, hexane/EtOAc) (Table 2; $76.9 \mathrm{mg}, 0.17 \mathrm{mmol}, 87 \%$ isolated yield). White solid. M.p. 106-108 ${ }^{\circ} \mathrm{C}$. IR (neat) 1093, $1161,1246,1278,1365,1423,1587,1681 \mathrm{~cm}^{-1} .{ }^{1} \mathbf{H}$ NMR (600 MHz, $\left.\mathrm{CDCl}_{3}\right) \delta 0.92(\mathrm{~s}, 3 \mathrm{H}), 1.19-$ $1.21(\mathrm{~m}, 2 \mathrm{H}), 1.27-1.40(\mathrm{~m}, 2 \mathrm{H}), 1.43(\mathrm{~s}, 9 \mathrm{H}), 1.64(\mathrm{dd}, J=14.4,3.6 \mathrm{~Hz}, 1 \mathrm{H}), 2.66(\mathrm{dd}, J=14.4,8.4$ $\mathrm{Hz}, 1 \mathrm{H}), 3.12-3.13(\mathrm{~m}, 2 \mathrm{H}), 3.53-3.54(\mathrm{~m}, 2 \mathrm{H}), 4.66$ (dd, $J=8.4,3.6 \mathrm{~Hz}, 1 \mathrm{H}), 7.20(\mathrm{~m}, 1 \mathrm{H}), 7.26-$ $7.29(\mathrm{~m}, 4 \mathrm{H}), 7.38(\mathrm{~d}, J=8.4 \mathrm{~Hz}, 2 \mathrm{H}), 7.91(\mathrm{~d}, J=8.4 \mathrm{~Hz}, 2 \mathrm{H}) .{ }^{13} \mathbf{C} \mathbf{N M R}\left(150.9 \mathrm{MHz}, \mathrm{CDCl}_{3}\right)$ $\delta 23.6,28.4,32.2,37.0,39.7,45.5,48.6,79.3,127.1,128.0,129.0,129.2,130.0,135.0,139.4,140.5$, 154.9, 198.3. HRMS-DART $(\mathrm{m} / \mathrm{z})$ : $[\mathrm{M}]^{+}$calcd for $\mathrm{C}_{26} \mathrm{H}_{32} \mathrm{ClNO}_{3}, 441.2065$; found, 441.2071.

tert-Butyl 4-[3-(4-Bromophenyl)-3-oxo-2-phenylpropyl]-4-methylpiperidine-1-carboxylate (4iaa)

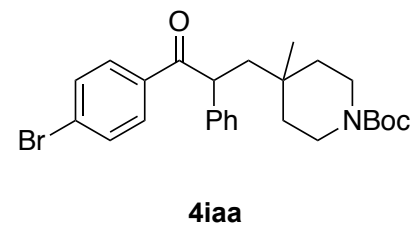

The product 4iaa was purified by flash chromatography on silica gel (98:2-94:6, hexane/EtOAc) (Table 2; $76.9 \mathrm{mg}, 0.16 \mathrm{mmol}, 79 \%$ isolated yield). White solid. M.p. $126-128{ }^{\circ} \mathrm{C}$. IR (neat) 1071, $1161,1246,1364,1423,1584,1682 \mathrm{~cm}^{-1} .{ }^{1} \mathbf{H}$ NMR (600 MHz, $\left.\mathrm{CDCl}_{3}\right) \delta 0.92(\mathrm{~s}, 3 \mathrm{H}), 1.19-1.22(\mathrm{~m}$, $2 \mathrm{H}), 1.27-1.34(\mathrm{~m}, 2 \mathrm{H}), 1.43(\mathrm{~s}, 9 \mathrm{H}), 1.63(\mathrm{dd}, J=13.8,3.6 \mathrm{~Hz}, 1 \mathrm{H}), 2.65(\mathrm{dd}, J=13.8,8.4 \mathrm{~Hz}, 1 \mathrm{H})$, $3.11-3.13(\mathrm{~m}, 2 \mathrm{H}), 3.52-3.54(\mathrm{~m}, 2 \mathrm{H}), 4.65(\mathrm{dd}, J=8.4,3.6 \mathrm{~Hz}, 1 \mathrm{H}), 7.19(\mathrm{~m}, 1 \mathrm{H}), 7.26-7.28(\mathrm{~m}$, $4 \mathrm{H}), 7.54(\mathrm{~d}, J=8.4 \mathrm{~Hz}, 2 \mathrm{H}), 7.83(\mathrm{~d}, J=8.4 \mathrm{~Hz}, 2 \mathrm{H}) .{ }^{13} \mathbf{C}$ NMR $\left(150.9 \mathrm{MHz}, \mathrm{CDCl}_{3}\right) \delta 23.6,28.4$, 
32.2, 36.9, 39.7, 45.5, 48.5, 79.3, 127.1, 128.0, 128.1, 129.1, 130.1, 131.9, 135.3, 140.4, 154.9, 198.4. HRMS-DART $(\mathrm{m} / \mathrm{z})$ : [M] $]^{+}$calcd for $\mathrm{C}_{26} \mathrm{H}_{32} \mathrm{BrNO}_{3}$, 485.1560; found, 485.1579.

tert-Butyl 4-[3-(3-Methoxyphenyl)-3-oxo-2-phenylpropyl]-4-methylpiperidine-1-carboxylate (4jaa)

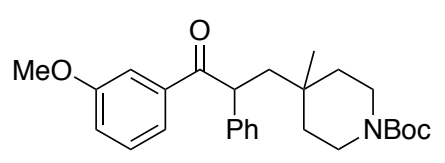

4jaa

The product $\mathbf{4 j a a}$ was purified by flash chromatography on silica gel (98:2-91:9, hexane/EtOAc) (Table 2; $74.4 \mathrm{mg}, 0.17 \mathrm{mmol}, 83 \%$ isolated yield). Colorless oil. IR (neat) 1162, 1261, 1365, 1426, $1453,1596,1685 \mathrm{~cm}^{-1} .{ }^{1} \mathbf{H}$ NMR $\left(600 \mathrm{~Hz}, \mathrm{CDCl}_{3}\right) \delta 0.93(\mathrm{~s}, 3 \mathrm{H}), 1.19-1.21(\mathrm{~m}, 2 \mathrm{H}), 1.32-1.39(\mathrm{~m}$, $2 \mathrm{H}), 1.43(\mathrm{~s}, 9 \mathrm{H}), 1.64(\mathrm{dd}, J=14.4,3.0 \mathrm{~Hz}, 1 \mathrm{H}), 2.67(\mathrm{dd}, J=14.4,8.4 \mathrm{~Hz}, 1 \mathrm{H}), 3.12-3.15(\mathrm{~m}, 2 \mathrm{H})$, 3.52-3.55 (m, 2H), $3.81(\mathrm{~s}, 3 \mathrm{H}), 4.72(\mathrm{dd}, J=8.4,3.0 \mathrm{~Hz}, 1 \mathrm{H}), 7.05(\mathrm{~m}, 1 \mathrm{H}), 7.18(\mathrm{~m}, 1 \mathrm{H}), 7.26-$ $7.33(\mathrm{~m}, 5 \mathrm{H}), 7.49(\mathrm{~m}, 1 \mathrm{H}), 7.58(\mathrm{~m}, 1 \mathrm{H}) .{ }^{13} \mathbf{C}$ NMR $\left(150.9 \mathrm{MHz}, \mathrm{CDCl}_{3}\right) \delta 23.7,28.5,32.2,37.0$, 39.7, 45.7, 48.6, 55.4, 79.2, 113.2, 119.3, 121.1, 126.9, 128.1, 129.0, 129.6, 138.1, 140.8, 154.9, 159.9, 199.4. HRMS-DART (m/z): [M] $]^{+}$calcd for $\mathrm{C}_{27} \mathrm{H}_{35} \mathrm{NO}_{4}, 437.2561$; found, 437.2554 .

tert-Butyl 4-Methyl-4-[3-oxo-2-phenyl-3-(pyridin-3-yl)propyl]piperidine-1-carboxylate (4kaa)

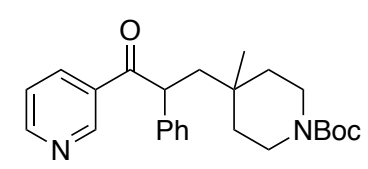

4kaa

The product 4kaa was purified by flash chromatography on silica gel (90:10-80:20, hexane/EtOAc) (Table 2; $71.9 \mathrm{mg}, 0.18 \mathrm{mmol}, 88 \%$ isolated yield). White solid. M.p. $117-121{ }^{\circ} \mathrm{C}$. IR (neat) 1160, 1246, 1278, 1365, 1418, 1453, 1584, $1684 \mathrm{~cm}^{-1} .{ }^{1} \mathbf{H}$ NMR (400 MHz, $\left.\mathrm{CDCl}_{3}\right) \delta 0.94$ (s, 3H), 1.20-1.24 (m, 2H), 1.29-1.33 (m, 2H), $1.43(\mathrm{~s}, 9 \mathrm{H}), 1.67$ (dd, J=14.0, 3.6 Hz, 1H), 2.67 (dd, $J=14.0,8.4 \mathrm{~Hz}, 1 \mathrm{H}), 3.09-3.15(\mathrm{~m}, 2 \mathrm{H}), 3.49-3.55(\mathrm{~m}, 2 \mathrm{H}), 4.67$ (dd, $J=8.4,3.6 \mathrm{~Hz}, 1 \mathrm{H}), 7.21$ (m, $1 \mathrm{H}), 7.26-7.29(\mathrm{~m}, 4 \mathrm{H}), 7.36(\mathrm{dd}, J=8.0,4.8 \mathrm{~Hz}, 1 \mathrm{H}), 8.22(\mathrm{dt}, J=8.0,1.6 \mathrm{~Hz}, 1 \mathrm{H}), 8.71(\mathrm{dd}, J=$ 4.8, 1.6 Hz, 1H), $9.21(\mathrm{~d}, J=1.6 \mathrm{~Hz}, 1 \mathrm{H}) . \delta^{\mathbf{1 3}} \mathbf{C} \mathbf{N M R}\left(150.9 \mathrm{MHz}, \mathrm{CDCl}_{3}\right) \delta 23.6,28.5,32.2,37.0$, 39.7, 45.2, 49.2, 79.3, 123.7, 127.3, 128.1, 129.3, 132.0, 136.2, 139.9, 149.9, 153.2, 154.9, 198.3. HRMS-DART $(\mathrm{m} / z)$ : [M] $]^{+}$calcd for $\mathrm{C}_{25} \mathrm{H}_{32} \mathrm{~N}_{2} \mathrm{O}_{3}, 408.2407$; found, 408.2407 .

tert-Butyl 4-Methyl-4-[3-oxo-2-phenyl-3-(thiophen-2-yl)propyl]piperidine-1-carboxylate (4laa)

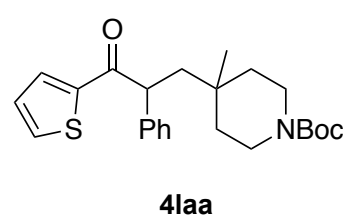

The product 4laa was purified by flash chromatography on silica gel (98:2-93:7, hexane/EtOAc) (Table 2; $71.1 \mathrm{mg}, 0.17 \mathrm{mmol}, 86 \%$ isolated yield). White solid. M.p. $152-154{ }^{\circ} \mathrm{C}$. IR (neat) 1160 , $1245,1278,1365,1413,1599,1682 \mathrm{~cm}^{-1} .{ }^{1} \mathbf{H}$ NMR (400 MHz, $\left.\mathrm{CDCl}_{3}\right) \delta 0.95(\mathrm{~s}, 3 \mathrm{H}), 1.21-1.27(\mathrm{~m}$, 
2H), 1.29-1.36 (m, 2H), $1.43(\mathrm{~s}, 9 \mathrm{H}), 1.65(\mathrm{dd}, J=14.4,3.2 \mathrm{~Hz}, 1 \mathrm{H}), 2.64(\mathrm{dd}, J=14.4,8.8 \mathrm{~Hz}, 1 \mathrm{H})$, $3.10-3.15(\mathrm{~m}, 2 \mathrm{H}), 3.47-3.55(\mathrm{~m}, 2 \mathrm{H}), 4.53(\mathrm{dd}, J=8.8,3.2 \mathrm{~Hz}, 1 \mathrm{H}), 7.09(\mathrm{~m}, 1 \mathrm{H}), 7.20(\mathrm{~m}, 1 \mathrm{H})$, 7.26-7.30 (m, 2H), 7.33-7.34 (m, 2H), $7.60(\mathrm{~m}, 1 \mathrm{H}), 7.78(\mathrm{~m}, 1 \mathrm{H}) .{ }^{13} \mathbf{C}$ NMR $\left(150.9 \mathrm{MHz}, \mathrm{CDCl}_{3}\right)$ $\delta$ 23.6, 28.5, 32.2, 37.0, 39.7, 45.3, 50.4, 79.2, 127.1, 128.0, 128.2, 129.0, 132.1, 133.9, 140.8, 143.8, 154.9, 192.4. HRMS-DART $(m / z)$ : $[\mathrm{M}]^{+}$calcd for $\mathrm{C}_{24} \mathrm{H}_{31} \mathrm{NO}_{3} \mathrm{~S}, 413.2019$; found, 413.2031 .

tert-Butyl 4-[3-(Benzofuran-2-yl)-3-oxo-2-phenylpropyl]-4-methylpiperidine-1-carboxylate (4maa)

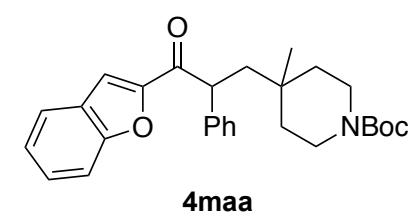

The product 4maa was purified by flash chromatography on silica gel (98:2-94:6, hexane/EtOAc) (Table 2; $39.4 \mathrm{mg}, 0.09 \mathrm{mmol}, 44 \%$ isolated yield). White solid. M.p. $162-165{ }^{\circ} \mathrm{C}$. IR (neat) 1089, $1161,1250,1281,1363,1425,1550,1667 \mathrm{~cm}^{-1} .{ }^{1} \mathbf{H}$ NMR (400 MHz, $\left.\mathrm{CDCl}_{3}\right) \delta 0.97(\mathrm{~s}, 3 \mathrm{H}), 1.23-$ $1.26(\mathrm{~m}, 2 \mathrm{H}), 1.34-1.38(\mathrm{~m}, 2 \mathrm{H}), 1.42(\mathrm{~s}, 9 \mathrm{H}), 1.70(\mathrm{dd}, J=14.0,3.2 \mathrm{~Hz}, 1 \mathrm{H}), 2.67(\mathrm{dd}, J=14.0,8.4$ Hz, 1H), 3.11-3.16 (m, 2H), 3.53-3.56 (m, 2H), 4.70 (dd, J=8.4, 3.2 Hz, 1H), 7.19 (m, 1H), 7.26$7.30(\mathrm{~m}, 4 \mathrm{H}), 7.38(\mathrm{~m}, 2 \mathrm{H}), 7.46(\mathrm{~m}, 1 \mathrm{H}), 7.56(\mathrm{~m}, 1 \mathrm{H}), 7.67(\mathrm{~m}, 1 \mathrm{H}) .{ }^{13} \mathbf{C} \mathbf{N M R}\left(150.9 \mathrm{MHz}, \mathrm{CDCl}_{3}\right)$ $\delta 23.6,28.4,32.2,37.0,39.7,44.6,49.2,79.2,112.5,113.6,123.3,123.9,127.1,127.2,128.3,129.0$, 140.1, 152.2, 154.9, 155.7, 190.6. HRMS-DART (m/z): [M] $]^{+}$calcd for $\mathrm{C}_{28} \mathrm{H}_{33} \mathrm{NO}_{4}, 447.2404$; found, 447.2397.

tert-Butyl 4-Methyl-4-[3-(naphthalen-2-yl)-3-oxo-2-phenylpropyl]piperidine-1-carboxylate (4naa)

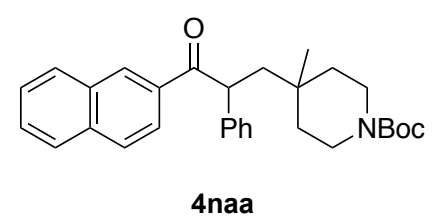

The product 4naa was purified by flash chromatography on silica gel (98:2-93:7, hexane/EtOAc) (Table 2; $75.0 \mathrm{mg}, 0.16 \mathrm{mmol}, 82 \%$ isolated yield). White solid. M.p. 129-132 ${ }^{\circ} \mathrm{C}$. IR (neat) 1123, $1162,1249,1278,1365,1424,1597,1678 \mathrm{~cm}^{-1} .{ }^{1} \mathbf{H}$ NMR (400 MHz, $\left.\mathrm{CDCl}_{3}\right) \delta 0.97(\mathrm{~s}, 3 \mathrm{H}), 1.21-$ $1.26(\mathrm{~m}, 2 \mathrm{H}), 1.31-1.36(\mathrm{~m}, 2 \mathrm{H}), 1.42(\mathrm{~s}, 9 \mathrm{H}), 1.69(\mathrm{dd}, J=14.4,3.2 \mathrm{~Hz}, 1 \mathrm{H}), 2.74(\mathrm{dd}, J=14.4,8.8$ $\mathrm{Hz}, 1 \mathrm{H}), 3.12-3.15$ (m, 2H), 3.54-3.57 (m, 2H), $4.90(\mathrm{dd}, J=8.8,3.2 \mathrm{~Hz}, 1 \mathrm{H}), 7.17$ (t, $J=7.2 \mathrm{~Hz}$, 1H), $7.28(\mathrm{t}, J=7.2 \mathrm{~Hz}, 2 \mathrm{H}), 7.36(\mathrm{~d}, J=7.2 \mathrm{~Hz}, 2 \mathrm{H}), 7.53-7.59(\mathrm{~m}, 2 \mathrm{H}), 7.82-7.87(\mathrm{~m}, 2 \mathrm{H}), 7.94$ $(\mathrm{m}, 1 \mathrm{H}), 8.03(\mathrm{~m}, 1 \mathrm{H}), 8.51(\mathrm{~s}, 1 \mathrm{H}) .{ }^{13} \mathbf{C} \mathbf{N M R}\left(150.9 \mathrm{MHz}, \mathrm{CDCl}_{3}\right) \delta 23.7,28.4,32.3,37.1,39.8$, 45.7, 48.6, 79.2, 123.6, 124.5, 126.8, 127.0, 127.7, 128.1, 128.50, 128.54, 129.1, 129.7, 130.1, 132.5, 134.1, 134.3, 135.5, 141.0, 154.9, 199.5. HRMS-DART $(\mathrm{m} / \mathrm{z})$ : $[\mathrm{M}]^{+}$calcd for $\mathrm{C}_{30} \mathrm{H}_{35} \mathrm{NO}_{3}, 457.2611$; found, 457.2622 .

\section{4,4-Dimethyl-1,2-diphenylpentan-1-one (4aab)}




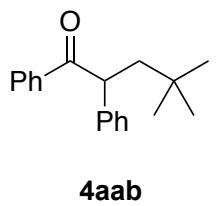

The product $4 \mathbf{a a b}$ was purified by flash chromatography on silica gel (100:0-95:5, hexane/EtOAc) (Table 2; $40.0 \mathrm{mg}, 0.15 \mathrm{mmol}, 75 \%$ isolated yield). The spectrum data of product 4 aab was consistent with the literature. ${ }^{3}$

\section{4-Methyl-1,2,4-triphenylpentan-1-one (4aac)}

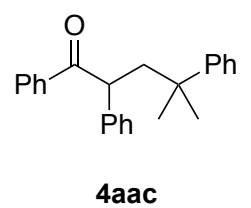

The product 4aac was purified by flash chromatography on silica gel (100:0-99:1, hexane/EtOAc) (Table 2; $37.9 \mathrm{mg}, 0.12 \mathrm{mmol}, 58 \%$ isolated yield). White solid. M.p. $73-75^{\circ} \mathrm{C}$. IR (neat) 1177, 1259, 1448, 1494, 1581, 1598, $1682 \mathrm{~cm}^{-1} .{ }^{1} \mathbf{H}$ NMR (600 MHz, $\left.\mathrm{CDCl}_{3}\right) \delta 1.26(\mathrm{~s}, 6 \mathrm{H}), 2.07(\mathrm{dd}, J=3.0$, $14.4 \mathrm{~Hz}, 1 \mathrm{H}), 2.99(\mathrm{dd}, J=8.2,14.4 \mathrm{~Hz}, 1 \mathrm{H}), 4.34(\mathrm{dd}, J=3.0,8.2 \mathrm{~Hz}, 1 \mathrm{H}), 7.08-7.09$ (m, 3H), 7.12 $(\mathrm{m}, 1 \mathrm{H}), 7.18-7.30(\mathrm{~m}, 6 \mathrm{H}), 7.43(\mathrm{~m}, 1 \mathrm{H}), 7.72-7.73(\mathrm{~m}, 2 \mathrm{H}) .{ }^{13} \mathbf{C ~ N M R}\left(150.9 \mathrm{MHz}, \mathrm{CDCl}_{3}\right) \delta 28.3$, 30.2, 38.6, 47.9, 50.0, 125.8, 126.2, 126.7, 128.0, 128.2, 128.3, 128.4, 128.8, 132.7, 136.7, 140.8, 199.9. HRMS-DART $(\mathrm{m} / \mathrm{z})$ : [M] ${ }^{+}$calcd for $\mathrm{C}_{24} \mathrm{H}_{24} \mathrm{O}$, 328.1820; found, 328.1801 .

\section{3-(1-Methylcyclopropyl)-1,2-diphenylpropan-1-one (4aad)}<smiles>CC1(CC(C(=O)c2ccccc2)c2ccccc2)CC1</smiles>

4aad

The product 4aad was purified by flash chromatography on silica gel (100:0-99:1, hexane/EtOAc) (Table 2; $21.1 \mathrm{mg}, 0.08 \mathrm{mmol}, 40 \%$ isolated yield). White solid. M.p. $73-75^{\circ} \mathrm{C}$. IR (neat) 1177,1259 , $1448,1494,1581,1598,1682 \mathrm{~cm}^{-1} .{ }^{1} \mathbf{H}$ NMR $\left(600 \mathrm{MHz}, \mathrm{CDCl}_{3}\right) \delta 0.06-0.12(\mathrm{~m}, 2 \mathrm{H}), 0.16(\mathrm{~m}, 1 \mathrm{H})$, $0.31(\mathrm{~m}, 1 \mathrm{H}), 1.01(\mathrm{~s}, 3 \mathrm{H}), 1.66(\mathrm{dd}, J=6.0,14.0 \mathrm{~Hz}, 1 \mathrm{H}), 2.41(\mathrm{dd}, J=7.8,14.0 \mathrm{~Hz}, 1 \mathrm{H}), 4.80(\mathrm{dd}$, $J=6.0,7.8 \mathrm{~Hz}, 1 \mathrm{H}), 7.18(\mathrm{~m}, 1 \mathrm{H}), 7.25-7.28(\mathrm{~m}, 2 \mathrm{H}), 7.31-7.33(\mathrm{~m}, 2 \mathrm{H}), 7.40-7.42(\mathrm{~m}, 2 \mathrm{H}), 7.49$ (m, 1H), 7.99-8.01 (m, 2H). ${ }^{13} \mathbf{C}$ NMR (150.9 MHz, $\left.\mathrm{CDCl}_{3}\right) \delta 13.1,13.4,14.4,23.3,43.5,51.6,126.9$, 128.2, 128.5, 128.6, 128.8, 132.8, 137.1, 140.2, 200.2. HRMS-DART $(\mathrm{m} / \mathrm{z})$ : [M] $]^{+}$calcd for $\mathrm{C}_{19} \mathrm{H}_{20} \mathrm{O}$, 264.1507; found, 264.1535.

\section{1,2-Diphenyl-3-(1-phenylcyclopentyl)propan-1-one (4aae)}

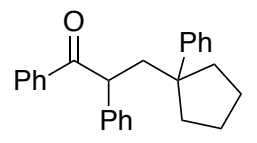


The product 4aae was purified by flash chromatography on silica gel (100:0-99:1, hexane/EtOAc) (Table 2; $31.9 \mathrm{mg}, 0.90 \mathrm{mmol}, 45 \%$ isolated yield). White solid. M.p. 111-113 ${ }^{\circ} \mathrm{C}$. IR (neat) 1218, 1356, 1446, 1493, 1581, 1597, $1682 \mathrm{~cm}^{-1} .{ }^{1} \mathbf{H}$ NMR (400 MHz, $\left.\mathrm{CDCl}_{3}\right) \delta 1.54(\mathrm{~s}, 2 \mathrm{H}), 1.66-1.76(\mathrm{~m}$, $4 \mathrm{H}), 1.78-1.86(\mathrm{~m}, 2 \mathrm{H}), 2.05(\mathrm{dd}, J=4.0,13.8 \mathrm{~Hz}, 1 \mathrm{H}), 2.89(\mathrm{dd}, J=7.2,13.8 \mathrm{~Hz}, 1 \mathrm{H}), 4.32(\mathrm{dd}, J$ $=4.0,7.2 \mathrm{~Hz}, 1 \mathrm{H}), 7.04-7.06(\mathrm{~m}, 2 \mathrm{H}), 7.11(\mathrm{~m}, 1 \mathrm{H}), 7.16-7.20(\mathrm{~m}, 3 \mathrm{H}), 7.24-7.32(\mathrm{~m}, 6 \mathrm{H}), 7.43(\mathrm{~m}$, 1H), 7.66-7.68 (m, 2H). ${ }^{13} \mathbf{C}$ NMR (150.9 MHz, $\left.\mathrm{CDCl}_{3}\right) \delta 22.7,22.9,37.7,38.2,45.5,50.1,51.6$, $125.8,126.7,127.2(\times 2 \mathrm{C}), 128.1,128.3,128.5,128.7,132.6,136.7,140.6,147.5,199.8$. HRMSDART $(\mathrm{m} / \mathrm{z}):[\mathrm{M}]^{+}$calcd for $\mathrm{C}_{26} \mathrm{H}_{26} \mathrm{O}, 354.1976$; found, 354.1969 .

\section{3-(1-Methylcyclohexyl)-1,2-diphenylpropan-1-one (4aaf)}

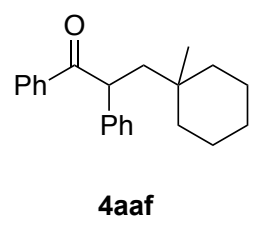

The product 4aaf was purified by flash chromatography on silica gel (100:0-99:1, hexane/EtOAc) (Table 2; $52.9 \mathrm{mg}, 0.17 \mathrm{mmol}, 86 \%$ isolated yield). White solid. M.p. $88-90{ }^{\circ} \mathrm{C}$. IR (neat) 1175,1215 , 1447, 1493, 1580, 1597, $1682 \mathrm{~cm}^{-1} .{ }^{1} \mathbf{H}$ NMR $\left(400 \mathrm{MHz}, \mathrm{CDCl}_{3}\right) \delta 0.85(\mathrm{~s}, 3 \mathrm{H}), 1.17(\mathrm{~m}, 1 \mathrm{H}), 1.22-$ $1.27(\mathrm{~m}, 4 \mathrm{H}), 1.32-1.43(\mathrm{~m}, 5 \mathrm{H}), 1.61(\mathrm{dd}, J=3.2,14.2 \mathrm{~Hz}, 1 \mathrm{H}), 2.63(\mathrm{dd}, J=8.8,14.2 \mathrm{~Hz}, 1 \mathrm{H})$, $4.75(\mathrm{dd}, J=3.2,8.8 \mathrm{~Hz}, 1 \mathrm{H}), 7.16(\mathrm{~m}, 1 \mathrm{H}), 7.24-7.28(\mathrm{~m}, 2 \mathrm{H}), 7.31-7.33(\mathrm{~m}, 2 \mathrm{H}), 7.39-7.42(\mathrm{~m}$, 2H), $7.47(\mathrm{~m}, 1 \mathrm{H}), 7.98-8.00(\mathrm{~m}, 2 \mathrm{H}) .{ }^{13} \mathbf{C} \mathbf{N M R}\left(150.9 \mathrm{MHz}, \mathrm{CDCl}_{3}\right) \delta 21.9,24.9,26.3,33.6,38.06$, $38.13,48.6,126.7,128.1,128.56,128.60,128.9,132.7,137.1,141.4,200.0$. HRMS-DART $(\mathrm{m} / z)$ : $[\mathrm{M}]^{+}$calcd for $\mathrm{C}_{22} \mathrm{H}_{26} \mathrm{O}, 306.1976$; found, 306.1967

\section{3-(1-Adamantanyl)-1,2-diphenylpropan-1-one (4aag)}

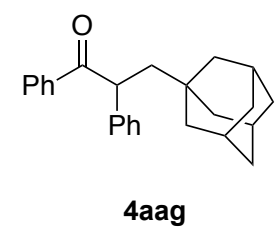

The product 4aag was purified by flash chromatography on silica gel (100:0-99:1, hexane/EtOAc) (Table $2 ; 48.9 \mathrm{mg}, 0.14 \mathrm{mmol}, 71 \%$ isolated yield). The spectrum data of product 4 aag was consistent with the literature. ${ }^{4}$

\section{Methyl 4-(3-Oxo-2,3-diphenylpropyl)bicyclo[2.2.2]octane-1-carboxylate (4aah)}

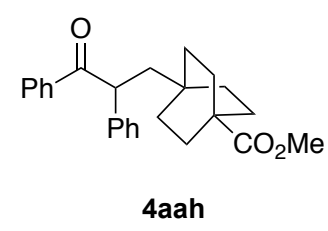

The product 4aah was purified by flash chromatography on silica gel (100:0-97.5:2.5, hexane/EtOAc) (Table 2; $50.1 \mathrm{mg}, 0.13 \mathrm{mmol}, 67 \%$ isolated yield). White solid. M.p. $125-127{ }^{\circ} \mathrm{C}$. IR (neat) 1234, 1447, 1493, 1581, 1597, 1681, $1723 \mathrm{~cm}^{-1} .{ }^{\mathbf{1}} \mathbf{H} \mathbf{N M R}\left(400 \mathrm{MHz}, \mathrm{CDCl}_{3}\right) \delta 1.29-1.36$ (m, 3H), 1.43-1.52 (m, 4H), 1.70-1.74 (m, 6H), 2.53 (dd, $J=9.2,14.2 \mathrm{~Hz}, 1 \mathrm{H}), 3.60$ (s, 3H), 4.70 
$(\mathrm{dd}, J=3.2,9.2 \mathrm{~Hz}, 1 \mathrm{H}), 7.18(\mathrm{~m}, 1 \mathrm{H}), 7.24-7.29(\mathrm{~m}, 4 \mathrm{H}), 7.39-7.43(\mathrm{~m}, 2 \mathrm{H}), 7.50(\mathrm{~m}, 1 \mathrm{H}), 7.97-$ $7.99(\mathrm{~m}, 2 \mathrm{H}) .{ }^{13} \mathbf{C} \mathbf{N M R}\left(150.9 \mathrm{MHz}, \mathrm{CDCl}_{3}\right) \delta 28.4,30.7,31.2,38.7,45.1,48.7,51.6,126.8,128.1$, $128.6(\times 2 \mathrm{C}), 128.9,132.9,136.8,140.9,178.3,199.6 \quad$ HRMS-DART $(\mathrm{m} / \mathrm{z}):[\mathrm{M}]^{+}$calcd for $\mathrm{C}_{25} \mathrm{H}_{28} \mathrm{O}_{3}, 376.2031$; found, 376.2044 .

\section{2,2-Dimethyl-5-oxo-4,5-diphenylpentyl Benzoate (4aai)}

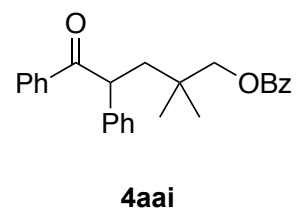

The product 4aai was purified by flash chromatography on silica gel (100:0-97.5:2.5, hexane/EtOAc) (Table 2; $60.5 \mathrm{mg}, 0.16 \mathrm{mmol}, 78 \%$ isolated yield). White solid. M.p. $70-72{ }^{\circ} \mathrm{C}$. IR (neat) 1112, 1270, 1372, 1449, 1598, 1682, $1717 \mathrm{~cm}^{-1} .{ }^{1} \mathbf{H}$ NMR (600 MHz, CDCl $) \delta 0.995(\mathrm{~s}, 3 \mathrm{H})$, 1.004 (s, 3H), 1.83 (dd, $J=4.2,14.4 \mathrm{~Hz}, 1 \mathrm{H}), 2.73$ (dd, $J=8.4,14.4 \mathrm{~Hz}, 1 \mathrm{H}), 4.04$ (dd, $J=11.2$, $30.6 \mathrm{~Hz}, 2 \mathrm{H}), 4.80$ (dd, $J=4.2,8.4 \mathrm{~Hz}, 1 \mathrm{H}), 7.16(\mathrm{~m}, 1 \mathrm{H}), 7.22-7.25(\mathrm{~m}, 2 \mathrm{H}), 7.30-7.32(\mathrm{~m}, 2 \mathrm{H})$, 7.36-7.38 (m, 2H), 7.40-7.43 (m, 2H), $7.47(\mathrm{~m}, 1 \mathrm{H}), 7.55(\mathrm{~m}, 1 \mathrm{H}), 7.96-8.00(\mathrm{~m}, 4 \mathrm{H}) .{ }^{13} \mathbf{C}$ NMR $\left(150.9 \mathrm{MHz}, \mathrm{CDCl}_{3}\right) \delta 24.7,25.4,34.7,42.7,49.1,72.4,126.9,128.1,128.3,128.56,128.59,129.0$, $129.5,130.3,132.8,132.9,136.7,140.5,166.5,199.4$ HRMS-DART $(\mathrm{m} / \mathrm{z}):[\mathrm{M}]^{+}$calcd for $\mathrm{C}_{26} \mathrm{H}_{26} \mathrm{O}_{3}, 386.1875$; found, 386.1862 .

\section{4,4-Dimethyl-1,2-diphenyl-5-[(tetrahydro-2H-pyran-2-yl)oxy]pentan-1-one (4aaj)}

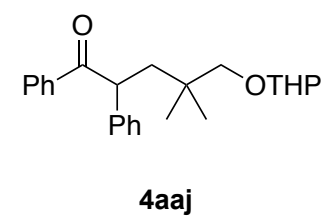

The product 4aaj was purified by flash chromatography on silica gel (100:0-97.5:2.5, hexane/EtOAc) (Table 2; $57.7 \mathrm{mg}, 0.16 \mathrm{mmol}, 79 \%$ isolated yield, 1:1 dr). White solid. M.p. 64$66^{\circ} \mathrm{C}$. IR (neat) $1201,1350,1382,1448,1493,1597,1683 \mathrm{~cm}^{-1} .{ }^{1} \mathbf{H}$ NMR (400 MHz, $\left.\mathrm{CDCl}_{3}\right) \delta$ $0.87-0.91(\mathrm{~m}, 6 \mathrm{H}), 1.47-1.78(\mathrm{~m}, 3 \mathrm{H}), 2.62(\mathrm{~m}, 1 \mathrm{H}), 3.03(\mathrm{~m}, 1 \mathrm{H}), 3.80(\mathrm{~m}, 1 \mathrm{H}), 4.38(\mathrm{~s}, 0.5 \times 1 \mathrm{H})$, $4.52(\mathrm{~s}, 0.5 \times 1 \mathrm{H}), 4.90(\mathrm{~m}, 1 \mathrm{H}), 7.16(\mathrm{~m}, 1 \mathrm{H}), 7.23-7.28(\mathrm{~m}, 2 \mathrm{H}), 7.31-7.34(\mathrm{~m}, 2 \mathrm{H}), 7.36-7.41(\mathrm{~m}$, 2H), 7.47 (m, 1H),8.00-8.02 (m, 2H). ${ }^{13} \mathbf{C} \mathbf{N M R}\left(100.5 \mathrm{MHz}, \mathrm{CDCl}_{3}\right) \delta 19.38,19.40,25.0,25.3$, $25.49,25.54,30.4,30.6,34.9,35.0,43.2,43.5,49.0,49.2,61.9,62.0,76.2(\times 2 \mathrm{C}), 98.9,99.1,126.7$ $(\times 2 \mathrm{C}), 128.19,128.24,128.4,128.5,128.6,128.7,128.8(\times 2 \mathrm{C}), 132.67,132.71,136.9,137.0,141.2$, 199.8, 200.0. HRMS-DART (m/z): [M] calcd for $\mathrm{C}_{24} \mathrm{H}_{30} \mathrm{O}_{3}, 366.2188$; found, 366.2207.

\section{2-Methyl-5-oxo-4,5-diphenylpentan-2-yl Acetate (4aak)}<smiles>CC(=O)OC(C)(C)CC(C(=O)c1ccccc1)c1ccccc1</smiles> 
The product 4aak was purified by flash chromatography on silica gel (100:0-95:5, hexane/EtOAc) (Table 2; $45.4 \mathrm{mg}, 0.15 \mathrm{mmol}, 73 \%$ isolated yield). Pale yellow oil. IR (neat) 1128, 1205, 1367, 1448, 1597, 1683, $1733 \mathrm{~cm}^{-1} .{ }^{1} \mathbf{H}$ NMR $\left(400 \mathrm{MHz}, \mathrm{CDCl}_{3}\right) \delta 1.44(\mathrm{~s}, 3 \mathrm{H}), 1.52$ (s, 3H), 1.72 (s, 3H), 2.15 $(\mathrm{dd}, J=3.6,14.8 \mathrm{~Hz}, 1 \mathrm{H}), 2.95(\mathrm{dd}, J=8.8,14.8 \mathrm{~Hz}, 1 \mathrm{H}), 4.87(\mathrm{dd}, J=3.6,8.8 \mathrm{~Hz}, 1 \mathrm{H}), 7.18(\mathrm{~m}$, 1H), 7.26-7.33 (m, 4H), 7.40-7.44 (m, 2H), 7.51 (m, 1H), 7.99-8.01 (m, 2H). ${ }^{13}$ C NMR (150.9 MHz, $\left.\mathrm{CDCl}_{3}\right) \delta 22.1,26.1,26.5,45.3,48.5,81.6,127.0,128.2,128.4,128.6,129.0,132.9,136.7,140.0$, 170.2, 199.2. HRMS-DART $(\mathrm{m} / \mathrm{z}):\left[\mathrm{M}+\mathrm{NH}_{4}\right]^{+}$calcd for $\mathrm{C}_{20} \mathrm{H}_{26} \mathrm{O}_{3}, 328.1907$; found, 328.1935.

\section{tert-Butyl 2-Methyl-2-(3-oxo-2,3-diphenylpropyl)pyrrolidine-1-carboxylate (4aal)}

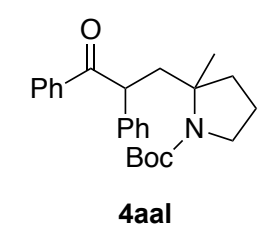

The product 4aal was purified by flash chromatography on silica gel (100:0-95:5, hexane/EtOAc) (Table 2; 72.3mg, $0.18 \mathrm{mmol}, 92 \%$ isolated yield, $1: 1 \mathrm{dr}$ ). Pale yellow oil. IR (neat) 1171, 1213, 1254, $1365,1388,1778,1597,1681 \mathrm{~cm}^{-1} .{ }^{1} \mathbf{H}$ NMR $\left(600 \mathrm{MHz}, \mathrm{CDCl}_{3}\right) \delta 1.27(\mathrm{~s}, 0.5 \times 3 \mathrm{H}), 1.30(\mathrm{~s}, 0.5 \times$ $3 \mathrm{H}), 1.36(\mathrm{~s}, 9 \mathrm{H}), 1.56-1.77(\mathrm{~m}, 4 \mathrm{H}), 21.7-2.38(\mathrm{~m}, 0.5 \times 2 \mathrm{H}), 2.87(\mathrm{~m}, 0.5 \times 1 \mathrm{H}), 3.02(\mathrm{~m}, 0.5 \times$ $1 \mathrm{H}), 3.21-3.34(\mathrm{~m}, 0.5 \times 2 \mathrm{H}), 3.40(\mathrm{~m}, 0.5 \times 1 \mathrm{H}), 3.60(\mathrm{~m}, 0.5 \times 1 \mathrm{H}), 4.65(\mathrm{~m}, 0.5 \times 1 \mathrm{H}), 4.86(\mathrm{~m}, 0.5$ $\times 1 \mathrm{H}), 7.16(\mathrm{~m}, 1 \mathrm{H}), 7.25-7.28(\mathrm{~m}, 3 \mathrm{H}), 7.33-7.41(\mathrm{~m}, 3 \mathrm{H}), 7.45(\mathrm{~m}, 1 \mathrm{H}), 7.95-8.01(\mathrm{~m}, 2 \mathrm{H}) .{ }^{13} \mathrm{C}$ NMR $\left(150.9 \mathrm{MHz}, \mathrm{CDCl}_{3}\right) \delta 21.3,21.5,21.6,21.7,25.0,25.4,26.5(\times 2 \mathrm{C}), 26.7(\times 2 \mathrm{C}), 28.37,28.40$, $28.55,28.56,39.06,39.14,39.3,39.6,42.1,42.2(\times 2 \mathrm{C}), 42.5,48.2,48.3,48.46,48.53,49.5(\times 2 \mathrm{C})$, $49.8(\times 2 \mathrm{C}), 62.4(\times 2 \mathrm{C}), 62.8(\times 2 \mathrm{C}), 78.46,78.53,79.5,79.6,126.6,126.78,126.82,127.0,128.1$, $128.2,128.34,128.36,128.38,128.40,128.50,128.54(\times 2 \mathrm{C}), 128.6(\times 2 \mathrm{C}), 128.77(\times 2 \mathrm{C}), 128.81$ $(\times 2 \mathrm{C}), 128.9,132.6,132.7,132.8,132.9,136.6(\times 2 \mathrm{C}), 136.9(\times 2 \mathrm{C}), 140.3(\times 2 \mathrm{C}), 140.7(\times 2 \mathrm{C}), 153.5$, 153.6, 154.3, 154.5, 199.6, 199.7, 199.8, 200.0. HRMS-DART $(m / z):[\mathrm{M}]^{+}$calcd for $\mathrm{C}_{25} \mathrm{H}_{31} \mathrm{NO}_{3}$, 393.2297; found, 393.2324.

\section{tert-Butyl Methyl(2-methyl-5-oxo-4,5-diphenylpentan-2-yl)carbamate (4aam)}

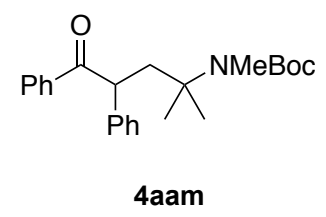

The product 4aam was purified by flash chromatography on silica gel (100:0-95:5, hexane/EtOAc) (Table 2; $64.7 \mathrm{mg}, 0.17 \mathrm{mmol}, 85 \%$ isolated yield). Pale yellow oil. IR (neat) 1117, 1157, 1239, 1363, 1447, 1597, $1680 \mathrm{~cm}^{-1} .{ }^{1} \mathbf{H}$ NMR $\left(600 \mathrm{MHz}, \mathrm{CDCl}_{3}\right) \delta 1.25(\mathrm{~s}, 3 \mathrm{H}), 1.26(\mathrm{~s}, 3 \mathrm{H}), 1.36(\mathrm{~s}, 9 \mathrm{H}), 2.36$ $(\mathrm{dd}, J=4.2,14.4 \mathrm{~Hz}, 1 \mathrm{H}), 2.78(\mathrm{~s}, 3 \mathrm{H}), 2.91(\mathrm{dd}, J=7.8,14.4 \mathrm{~Hz}, 1 \mathrm{H}), 4.75(\mathrm{dd}, J=4.2,7.8 \mathrm{~Hz}$, 1H), $7.16(\mathrm{~m}, 1 \mathrm{H}), 7.23-7.26(\mathrm{~m}, 2 \mathrm{H}), 7.30-7.31(\mathrm{~m}, 2 \mathrm{H}), 7.38-7.40(\mathrm{~m}, 2 \mathrm{H}), 7.48(\mathrm{~m}, 1 \mathrm{H}), 7.98-$ $7.99(\mathrm{~m}, 2 \mathrm{H}) .{ }^{13} \mathrm{C}$ NMR $\left(150.9 \mathrm{MHz}, \mathrm{CDCl}_{3}\right) \delta 28.3,28.4,28.8,32.0,44.3,49.4,57.9,79.5,126.8$, $128.3,128.5,128.6,128.8,132.8,136.8,140.5,156.0,199.8$. HRMS-DART $(\mathrm{m} / \mathrm{z}):[\mathrm{M}]^{+}$calcd for $\mathrm{C}_{24} \mathrm{H}_{31} \mathrm{NO}_{3}, 381.2297$; found, 381.2316. 


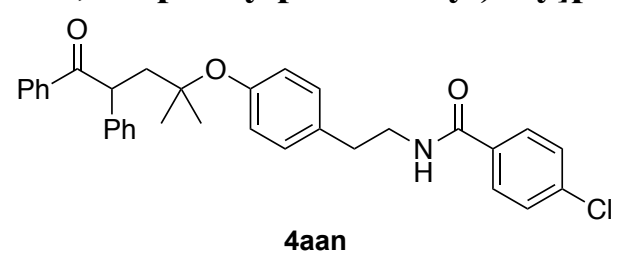

The product 4aan was purified by flash chromatography on silica gel (100:0-80:20, hexane/EtOAc) (Table 2; $102.6 \mathrm{mg}, 0.20 \mathrm{mmol}, 98 \%$ isolated yield). White solid. M.p. $125-127^{\circ} \mathrm{C}$. IR (neat) 1130 , 1220, 1307, 1486, 1506, 1532, 1642, $1675 \mathrm{~cm}^{-1} .{ }^{1} \mathbf{H}$ NMR (600 MHz, $\left.\mathrm{CDCl}_{3}\right) \delta 1.20(\mathrm{~s}, 3 \mathrm{H}), 1.24(\mathrm{~s}$, $3 \mathrm{H}), 2.08(\mathrm{dd}, J=3.0,14.4 \mathrm{~Hz}, 1 \mathrm{H}), 2.86(\mathrm{t}, 2 \mathrm{H}, J=6.8 \mathrm{~Hz}), 3.04(\mathrm{dd}, J=6.0,14.4 \mathrm{~Hz}, 1 \mathrm{H}), 3.66$ $(\mathrm{dd}, J=6.8,12.6 \mathrm{~Hz}, 2 \mathrm{H}), 5.15(\mathrm{~m}, 1 \mathrm{H}), 6.02$ (brs, 1H), 6.84-6.86 (m, 2H), 7.06-7.07 (m, 2H), 7.19 (m, 1H), 7.26-7.29 (m, 4H), 7.35-7.42 (m, 5H), $7.50(\mathrm{~m}, 1 \mathrm{H}), 7.59-7.61(\mathrm{~m}, 2 \mathrm{H}), 8.03-8.04(\mathrm{~m}, 2 \mathrm{H})$. ${ }^{13} \mathbf{C}$ NMR $\left(100.5 \mathrm{MHz}, \mathrm{CDCl}_{3}\right) \delta 27.1,17.3,34.8,41.2,46.0,48.5,79.9,124.0,126.9,128.2,128.3$, 128.6, 128.7, 128.8, 128.9, 129.2, 133.0, 132.8, 133.5, 136.9, 137.6, 140.4, 153.8, 166.3, 199.9. HRMS-DART $(\mathrm{m} / \mathrm{z}):[\mathrm{M}]^{+}$calcd for $\mathrm{C}_{33} \mathrm{H}_{32} \mathrm{ClNO}_{3}, 525.2063$; found, 525.2070

\section{7-(2,5-Dimethylphenoxy)-4,4-dimethyl-1,2-diphenylheptan-1-ol (4aao')}

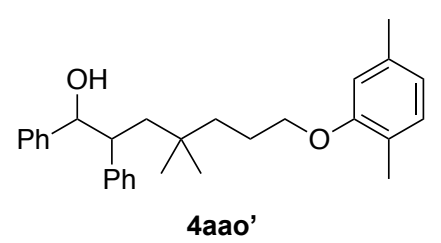

The product 4aao' was purified by flash chromatography on silica gel (100:0-95:5, hexane/EtOAc) (Table 2; $61.9 \mathrm{mg}, 0.15 \mathrm{mmol}, 74 \%$ isolated yield in 2 steps, 14:1 d.r.). Colorless oil. IR (neat) 1129, $1156,1263,1387,1452,1508,1584,1614 \mathrm{~cm}^{-1}$. ${ }^{1} \mathbf{H} \mathbf{~ N M R}\left(400 \mathrm{MHz}, \mathrm{CDCl}_{3}\right.$, major isomer) $\delta 0.58$ $(\mathrm{s}, 3 \mathrm{H}), 0.61(\mathrm{~s}, 3 \mathrm{H}), 1.04-1.21(\mathrm{~m}, 2 \mathrm{H}), 1.34-1.41(\mathrm{~m}, 2 \mathrm{H}), 1.45(\mathrm{dd}, J=2.4,14.0 \mathrm{~Hz}, 1 \mathrm{H}), 1.76$ $(\mathrm{dd}, J=5.6,8.4 \mathrm{~Hz}, 2 \mathrm{H}), 2.16(\mathrm{~s}, 3 \mathrm{H}), 2.31(\mathrm{~s}, 3 \mathrm{H}), 2.98(\mathrm{~m}, 1 \mathrm{H}), 3.62-3.67(\mathrm{~m}, 2 \mathrm{H}), 4.61(\mathrm{dd}, 1 \mathrm{H}$, $J=2.4,8.4 \mathrm{~Hz}), 6.54(\mathrm{~s}, 1 \mathrm{H}), 6.65(\mathrm{~m}, 1 \mathrm{H}), 7.00(\mathrm{~m}, 1 \mathrm{H}), 7.23-7.34(\mathrm{~m}, 10 \mathrm{H}) .{ }^{13} \mathrm{C}$ NMR $(150.9$ $\mathrm{MHz}, \mathrm{CDCl}_{3}$, major isomer) $\delta 15.8,21.4,23.9,27.8,27.9,33.3,38.1,42.7,50.5,68.4,79.7,112.0$, $120.5,123.5,126.8,127.3,127.8,128.2,128.5,129.2,130.2,136.4,142.6,142.9,157.1$. HRMSDART $(\mathrm{m} / \mathrm{z}):[\mathrm{M}]^{+}$calcd for $\mathrm{C}_{29} \mathrm{H}_{36} \mathrm{O}_{2}, 416.2708$; found, 416.2712 .

tert-Butyl 4-[2-(3,5-Dimethylphenyl)-3-oxo-3-phenylpropyl]-4-methylpiperidine-1-carboxylate (4aba)

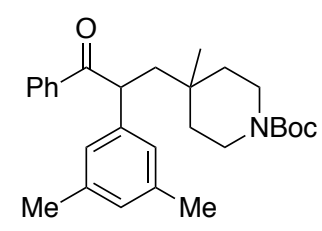

4aba

The product 4aba was purified by flash chromatography on silica gel (100:0-95:5, hexane/EtOAc) (Table 2; $70.8 \mathrm{mg}, 0.16 \mathrm{mmol}, 81 \%$ isolated yield). Pale yellow oil. IR (neat) 1159, 1245, 1278, 1364, 1421, 1447, 1597, $1683 \mathrm{~cm}^{-1} .{ }^{1} \mathbf{H}$ NMR (400 MHz, $\left.\mathrm{CDCl}_{3}\right) \delta 0.92(\mathrm{~s}, 3 \mathrm{H}), 1.19(\mathrm{~m}, 1 \mathrm{H}), 1.26-1.41$ $(\mathrm{m}, 4 \mathrm{H}), 1.43(\mathrm{~s}, 9 \mathrm{H}), 1.59(\mathrm{~m}, 1 \mathrm{H}), 2.26(\mathrm{~s}, 6 \mathrm{H}), 2.69(\mathrm{dd}, J=8.8,9.4 \mathrm{~Hz}, 1 \mathrm{H}), 3.12-3.14(\mathrm{~m}, 2 \mathrm{H})$, 
3.53-3.56 (m, 2H), $4.65(\mathrm{~m}, 1 \mathrm{H}), 6.81(\mathrm{~s}, 1 \mathrm{H}), 6.89(\mathrm{~s}, 2 \mathrm{H}), 7.40-7.43(\mathrm{~m}, 2 \mathrm{H}), 7.50(\mathrm{~m}, 1 \mathrm{H}), 7.97-$ $8.00(\mathrm{~m}, 2 \mathrm{H}) .{ }^{13} \mathbf{C} \mathbf{N M R}\left(150.9 \mathrm{MHz}, \mathrm{CDCl}_{3}\right) \delta 21.3,23.7,28.4,32.2,37.0,48.4,79.2,125.7(\times 2 \mathrm{C})$, 128.59, 128.62, 132.8, 136.9, 138.5, 140.7, 154.9, 199.6. HRMS-DART $(m / z):[\mathrm{M}+\mathrm{H}]^{+}$calcd for $\mathrm{C}_{21} \mathrm{H}_{20} \mathrm{O}_{2}, 436.2846$; found, 436.2864 .

tert-Butyl 4-[2-(4-Methoxyphenyl)-3-oxo-3-phenylpropyl]-4-methylpiperidine-1-carboxylate (4aca)

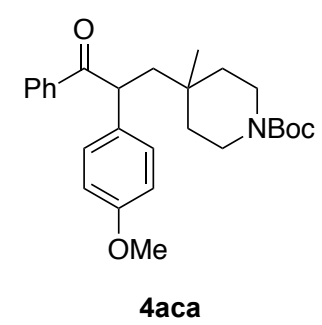

The product 4aca was purified by flash chromatography on silica gel (100:0-90:10, hexane/EtOAc) (Table 2; $70.9 \mathrm{mg}, 0.16 \mathrm{mmol}, 81 \%$ isolated yield). Pale yellow oil. IR (neat) 1159, 1248, 1364, 1422, 1509, 1608,1879, $1747 \mathrm{~cm}^{-1} .{ }^{1} \mathbf{H}$ NMR (600 MHz, $\left.\mathrm{CDCl}_{3}\right) \delta 0.93$ (s, 3H), $1.19-$ $1.40(\mathrm{~m}, 4 \mathrm{H}), 1.43(\mathrm{~s}, 9 \mathrm{H}), 1.63(\mathrm{~m}, 1 \mathrm{H}), 2.62(\mathrm{dd}, J=8.4,14.2 \mathrm{~Hz}, 1 \mathrm{H}), 3.12-3.14(\mathrm{~m}, 2 \mathrm{H}), 3.51-$ $3.53(\mathrm{~m}, 2 \mathrm{H}), 3.74(\mathrm{~s}, 3 \mathrm{H}), 4.69(\mathrm{dd}, J=3.4,8.2 \mathrm{~Hz}, 1 \mathrm{H}), 6.79-6.81(\mathrm{~m}, 2 \mathrm{H}), 7.20-7.22(\mathrm{~m}, 2 \mathrm{H})$, 7.40-7.42 (m, 2H), $7.50(\mathrm{~m}, 1 \mathrm{H}), 7.96-7.98(\mathrm{~m}, 2 \mathrm{H}) .{ }^{13} \mathbf{C} \mathbf{N M R}\left(150.9 \mathrm{MHz}, \mathrm{CDCl}_{3}\right) \delta 23.7,28.4$, 32.1, 37.0, 39.3, 40.1, 45.5, 47.5, 55.1, 79.1, 114.4, 128.5, 128.6, 129.0, 132.7, 132.8, 136.7, 154.9, 158.8, 199.7. HRMS-DART $(\mathrm{m} / \mathrm{z})$ : [M] $]^{+}$calcd for $\mathrm{C}_{27} \mathrm{H}_{35} \mathrm{NO}_{4}, 437.2559$; found,437.2570.

tert-Butyl 4-\{2-[4-(Benzyloxy)phenyl]-3-oxo-3-phenylpropyl\}-4-methylpiperidine-1carboxylate (4ada)

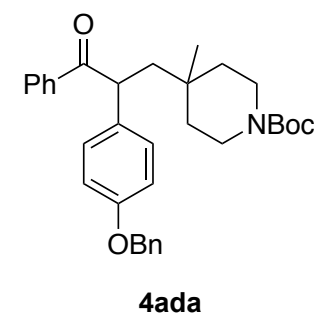

The product 4ada was purified by flash chromatography on silica gel (100:0-90:10, hexane/EtOAc) (Table 2; $85.3 \mathrm{mg}, 0.17 \mathrm{mmol}, 83 \%$ isolated yield). White solid. M.p. $137-139^{\circ} \mathrm{C}$. IR (neat) 1161, 1246, 1365, 1428, 1508, 1607, 1670, 1687, $1745 \mathrm{~cm}^{-1} .{ }^{1} \mathbf{H} \mathbf{N M R}\left(400 \mathrm{MHz}, \mathrm{CDCl}_{3}\right)$ $\delta 0.93(\mathrm{~s}, 3 \mathrm{H}), 1.18-1.42(\mathrm{~m}, 4 \mathrm{H}), 1.43(\mathrm{~s}, 9 \mathrm{H}), 1.63(\mathrm{dd}, J=3.2,14.2 \mathrm{~Hz}, 1 \mathrm{H}), 2.63(\mathrm{dd}, J=8.2$, $14.2 \mathrm{~Hz}, 1 \mathrm{H}), 3.10-3.16(\mathrm{~m}, 2 \mathrm{H}), 3.51-3.54(\mathrm{~m}, 2 \mathrm{H}), 4.69(\mathrm{dd}, 1 \mathrm{H}, J=3.2,8.2 \mathrm{~Hz}), 4.99(\mathrm{~s}, 2 \mathrm{H})$, 6.87-6.89 (m, 2H), 7.20-7.22 (m, 2H), 7.34-7.43 (m, 7H), $7.50(\mathrm{~m}, 1 \mathrm{H}), 7.96-7.98(\mathrm{~m}, 2 \mathrm{H}) .{ }^{13} \mathbf{C}$ NMR (150.9 MHz, $\left.\mathrm{CDCl}_{3}\right) \delta 23.7,28.4,32.2,37.0,39.8,45.6,47.5,70.0,79.2,115.3,127.4,128.0$, $128.55,128.58,128.62,129.1,132.9,133.0,136.7,136.9,154.9,157.8,199.7$. HRMS-DART $(\mathrm{m} / z)$ : $[\mathrm{M}]^{+}$calcd for $\mathrm{C}_{33} \mathrm{H}_{39} \mathrm{NO}_{4}, 513.2872$; found, 513.2870.

tert-Butyl 4-[2-(4-Bromophenyl)-3-hydroxy-3-phenylpropyl]-4-methylpiperidine-1-carboxylate (4aea') 


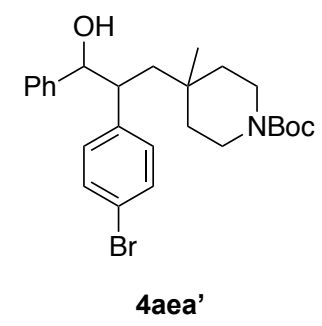

The product 4aea' was purified by flash chromatography on silica gel (100:0-80:20, hexane/EtOAc) (Table 2; $96.7 \mathrm{mg}, 0.20 \mathrm{mmol}$, 99\% isolated yield in 2 steps, 25:1 d.r.). Colorless oil. IR (neat) 1159, 1216, 1249, 1277, 1366, 1427, 1487, $1668 \mathrm{~cm}^{-1} .{ }^{\mathbf{1}} \mathbf{H} \mathbf{~ N M R}\left(600 \mathrm{MHz}, \mathrm{CDCl}_{3}\right.$, major isomer) $\delta 0.63(\mathrm{~s}, 3 \mathrm{H}), 1.01-1.14(\mathrm{~m}, 4 \mathrm{H}), 1.40(\mathrm{~s}, 9 \mathrm{H}), 1.52(\mathrm{~m}, 1 \mathrm{H}), 1.76(\mathrm{~m}, 1 \mathrm{H}), 2.94-2.98(\mathrm{~m}$, 2H), $3.05(\mathrm{~m}, 1 \mathrm{H}), 3.23$ (brs, 2H), $4.62(\mathrm{~m}, 1 \mathrm{H}), 7.11-7.13(\mathrm{~m}, 2 \mathrm{H}), 7.22-7.24(\mathrm{~m}, 2 \mathrm{H}), 7.27-7.33(\mathrm{~m}$, 3H), 7.42-7.43 (m, 2H). ${ }^{13} \mathbf{C}$ NMR (150.9 MHz, $\left.\mathrm{CDCl}_{3}\right) \delta$ 23.7, 28.4, 32.0, 37.0, 39.5, 43.2, 48.9, $79.1,79.3,120.4,126.9,127.8,128.2,130.8,131.3,141.8,142.3,154.8$. HRMS-DART $(\mathrm{m} / z)$ : $[\mathrm{M}+\mathrm{H}]^{+}$calcd for $\mathrm{C}_{26} \mathrm{H}_{35} \mathrm{BrNO}_{3}, 488.1795$; found, 488.1809 .

tert-Butyl 4-[2-(Benzo $[d][1,3]$ dioxol-5-yl)-3-oxo-3-phenylpropyl]-4-methylpiperidine-1carboxylate (4afa)

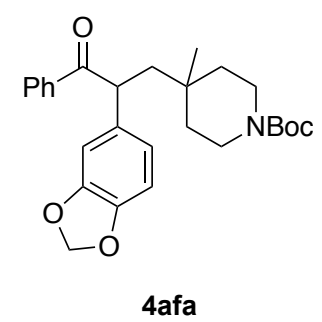

The product 4afa was purified by flash chromatography on silica gel (100:0-97.5:2.5, hexane/EtOAc) (Table 2; $75.0 \mathrm{mg}, 0.17 \mathrm{mmol}, 83 \%$ isolated yield). White solid. M.p. $137-139^{\circ} \mathrm{C}$. IR (neat) 1160, 1220, 1245, 1363, 1422, 1489, 1595, 1668, $1683 \mathrm{~cm}^{-1} .{ }^{\mathbf{1}} \mathbf{H} \mathbf{N M R}\left(400 \mathrm{MHz}, \mathrm{CDCl}_{3}\right)$ $\delta 0.92(\mathrm{~s}, 3 \mathrm{H}), 1.18-1.39(\mathrm{~m}, 4 \mathrm{H}), 1.43(\mathrm{~s}, 9 \mathrm{H}), 1.62(\mathrm{dd}, J=3.2,14.2 \mathrm{~Hz}, 1 \mathrm{H}), 2.60(\mathrm{dd}, J=8.2$, $14.2 \mathrm{~Hz}, 1 \mathrm{H}), 3.08-3.17(\mathrm{~m}, 2 \mathrm{H}), 3.52-3.55(\mathrm{~m}, 2 \mathrm{H}), 4.65$ (dd, $J=3.2,8.2 \mathrm{~Hz}, 1 \mathrm{H}), 5.89-5.90$ (m, 2H), 6.69-6.80 (m, 3H), 7.40-7.44 (m, 2H), 7.51 (m, 1H), 7.96-7.98 (m, 2H). ${ }^{13}$ C NMR (100.5 MHz, $\left.\mathrm{CDCl}_{3}\right) \delta 23.6,28.4,32.1,37.0,39.7,40.1,45.6,47.9,79.2,101.1,108.3,108.6,121.3,128.57$, 128.65, 133.0, 134.5, 136.6, 146.5, 148.1, 154.9, 199.5. HRMS-DART $(\mathrm{m} / z):[\mathrm{M}]^{+}$calcd for $\mathrm{C}_{27} \mathrm{H}_{33} \mathrm{NO}_{5}, 451.2351$; found, 451.2365 .

tert-Butyl 4-[2-(6-Methoxynaphthalen-2-yl)-3-oxo-3-phenylpropyl]-4-methylpiperidine-1carboxylate (4aga)

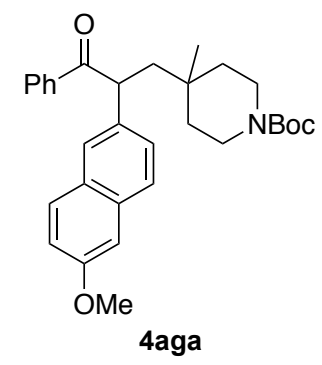


The product 4aga was purified by flash chromatography on silica gel (100:0-90:10, hexane/EtOAc) (Table 2; $91.5 \mathrm{mg}, 0.19 \mathrm{mmol}$, 94\% isolated yield). White solid. M.p. $116-118^{\circ} \mathrm{C}$. IR (neat) 1159, 1213, 1248, 1387, 1419, 1504, 1604, 1629, 1691 cm ${ }^{-1} .{ }^{\mathbf{1}} \mathbf{H} \mathbf{N M R}\left(400 \mathrm{MHz}, \mathrm{CDCl}_{3}\right)$ $\delta 0.96(\mathrm{~s}, 3 \mathrm{H}), 1.26-1.42(\mathrm{~m}, 4 \mathrm{H}), 1.42(\mathrm{~s}, 9 \mathrm{H}), 1.72(\mathrm{~m}, 1 \mathrm{H}), 2.74(\mathrm{dd}, J=8.2,13.8 \mathrm{~Hz}, 1 \mathrm{H}), 3.12^{-}$ $3.16(\mathrm{~m}, 2 \mathrm{H}), 3.52-3.56(\mathrm{~m}, 2 \mathrm{H}), 3.88(\mathrm{~s}, 3 \mathrm{H}), 4.86(\mathrm{dd}, J=3.2,5.0 \mathrm{~Hz}, 1 \mathrm{H}), 7.06(\mathrm{~s}, 1 \mathrm{H}), 7.10(\mathrm{~m}$, 1H), 7.37-7.43 (m, 3H), $7.48(\mathrm{~s}, 1 \mathrm{H}), 7.64-7.69(\mathrm{~m}, 3 \mathrm{H}), 8.00-8.02(\mathrm{~m}, 2 \mathrm{H}) .{ }^{13} \mathbf{C}$ NMR $(150.9 \mathrm{MHz}$, $\left.\mathrm{CDCl}_{3}\right) \delta 23.7,28.4,32.2,37.0,39.3,40.2,45.6,48.4,55.3,79.2,105.5,119.1,123.5,126.6,127.7$, 128.6, 129.1, 129.2, 132.9, 133.5, 134.2, 135.9, 136.8, 154.9, 157.7, 199.6. HRMS-DART $(\mathrm{m} / z)$ : $[\mathrm{M}]^{+}$calcd for $\mathrm{C}_{31} \mathrm{H}_{37} \mathrm{NO}_{4}, 487.2715$; found, 487.2713.

tert-Butyl

4-Methyl-4-[3-oxo-3-phenyl-2-(thiophen-2-yl)propyl]piperidine-1-carboxylate (4aha)

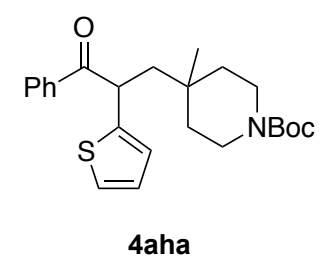

The product 4aha was purified by flash chromatography on silica gel (100:0-90:10, hexane/EtOAc) (Table 2; $49.1 \mathrm{mg}, 0.12 \mathrm{mmol}, 59 \%$ isolated yield). White solid. M.p. $128-130{ }^{\circ} \mathrm{C}$. IR (neat) 1147, 1230, 1255, 1279, 1366, 1416, 1474, 1596, $1672 \mathrm{~cm}^{-1} .{ }^{1} \mathbf{H}$ NMR $\left(400 \mathrm{MHz}, \mathrm{CDCl}_{3}\right)$ $\delta 0.94(\mathrm{~s}, 3 \mathrm{H}), 1.16-1.39(\mathrm{~m}, 4 \mathrm{H}), 1.43(\mathrm{~s}, 9 \mathrm{H}), 1.80(\mathrm{dd}, J=3.8,14.2 \mathrm{~Hz}, 1 \mathrm{H}), 2.63(\mathrm{dd}, J=8.2$, $14.2 \mathrm{~Hz}, 1 \mathrm{H}), 3.08-3.18(\mathrm{~m}, 2 \mathrm{H}), 3.54-3.57(\mathrm{~m}, 2 \mathrm{H}), 5.04(\mathrm{dd}, J=3.8,8.2 \mathrm{~Hz}, 1 \mathrm{H}), 6.87-6.89$ (m, 2H), $7.15(\mathrm{~m}, 1 \mathrm{H}), 7.44-7.47(\mathrm{~m}, 2 \mathrm{H}), 7.55(\mathrm{~m}, 1 \mathrm{H}), 8.00-8.02(\mathrm{~m}, 2 \mathrm{H}) .{ }^{13} \mathbf{C}$ NMR (150.9 MHz, $\left.\mathrm{CDCl}_{3}\right) \delta 23.6,28.4,32.2,36.9,39.2,40.1,42.9,46.3,79.2,124.8,125.3,127.0,128.68,128.74$, 133.2, 136.1, 143.2, 154.9, 198.4. HRMS-DART (m/z): [M] $]^{+}$calcd for $\mathrm{C}_{24} \mathrm{H}_{31} \mathrm{NO}_{3}, 413.2017$; found, 413.2007.

tert-Butyl 4-(2-Cyano-3-oxo-3-phenylpropyl)-4-methylpiperidine-1-carboxylate (4aia)

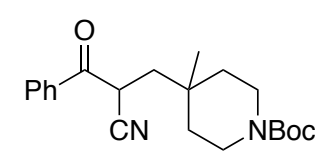

4aia

The product 4aia was purified by flash chromatography on silica gel (100:0-80:20, hexane/EtOAc) (Table 2; 37.8mg, $0.11 \mathrm{mmol}, 53 \%$ isolated yield). Colorless oil. IR (neat) 1161, 1250, 1366, 1392, 1425, 1597, $1692 \mathrm{~cm}^{-1} .{ }^{1} \mathbf{H}$ NMR (400 MHz, $\left.\mathrm{CDCl}_{3}\right) \delta 1.12(\mathrm{~s}, 3 \mathrm{H}), 1.39-1.44(\mathrm{~m}, 4 \mathrm{H}), 1.45(\mathrm{~s}, 9 \mathrm{H})$, 2.03-2.05 (m, 2H), 3.12-3.19 (m, 2H), 3.63-3.67 (m, 2H), $4.33(\mathrm{~m}, 1 \mathrm{H}), 7.53-7.57(\mathrm{~m}, 2 \mathrm{H}), 7.67$ (m, 1H), 7.97-7.99 (m, 2H). ${ }^{13} \mathbf{C}$ NMR (150.9 MHz, $\left.\mathrm{CDCl}_{3}\right) \delta 22.7,28.4,32.2,34.3,36.6,39.4,41.2$, 79.6, $118.3,128.8,129.2,133.7,134.6,154.8,190.6$. HRMS-DART $(\mathrm{m} / \mathrm{z}):[\mathrm{M}]^{+}$calcd for $\mathrm{C}_{21} \mathrm{H}_{28} \mathrm{~N}_{2} \mathrm{O}_{3}, 356.2093$; found, 356.2112 . 


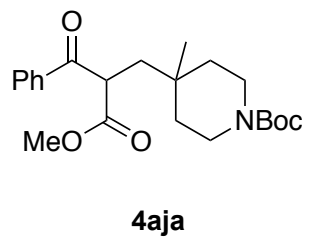

The product 4aja was purified by flash chromatography on silica gel (100:0-80:20, hexane/EtOAc) (Table 2; $46.7 \mathrm{mg}, 0.12 \mathrm{mmol}, 60 \%$ isolated yield). Colorless oil. IR (neat) 1161, 1247, 1278, 1365, 1424, 1597, 1686, $1741 \mathrm{~cm}^{-1} .{ }^{1} \mathbf{H}$ NMR $\left(600 \mathrm{MHz}, \mathrm{CDCl}_{3}\right) \delta 0.93(\mathrm{~s}, 3 \mathrm{H}), 1.28-1.40(\mathrm{~m}, 4 \mathrm{H}), 1.44$ (s, 9H), 2.07-2.13 (m, 2H), 3.08-3.18 (m, 2H), 3.55-3.64 (m, 2H), $3.68(\mathrm{~s}, 3 \mathrm{H}), 4.43,(\mathrm{~m}, 1 \mathrm{H}), 7.48-$ $7.52(\mathrm{~m}, 2 \mathrm{H}), 7.60(\mathrm{~m}, 1 \mathrm{H}), 7.99-8.01(\mathrm{~m}, 2 \mathrm{H}) .{ }^{13} \mathbf{C} \mathbf{N M R}\left(150.9 \mathrm{MHz}, \mathrm{CDCl}_{3}\right) \delta 23.0,28.4,31.9$, 40.2 (×2C), 49.4, 52.68, 52.72, 79.3, 128.7, 128.78, 128.85, 133.6, 136.8, 154.8, 170.8, 195.0 . HRMS-DART (m/z): [M] $]^{+}$calcd for $\mathrm{C}_{22} \mathrm{H}_{31} \mathrm{NO}_{5}, 389.2195$; found, 389.2203. 


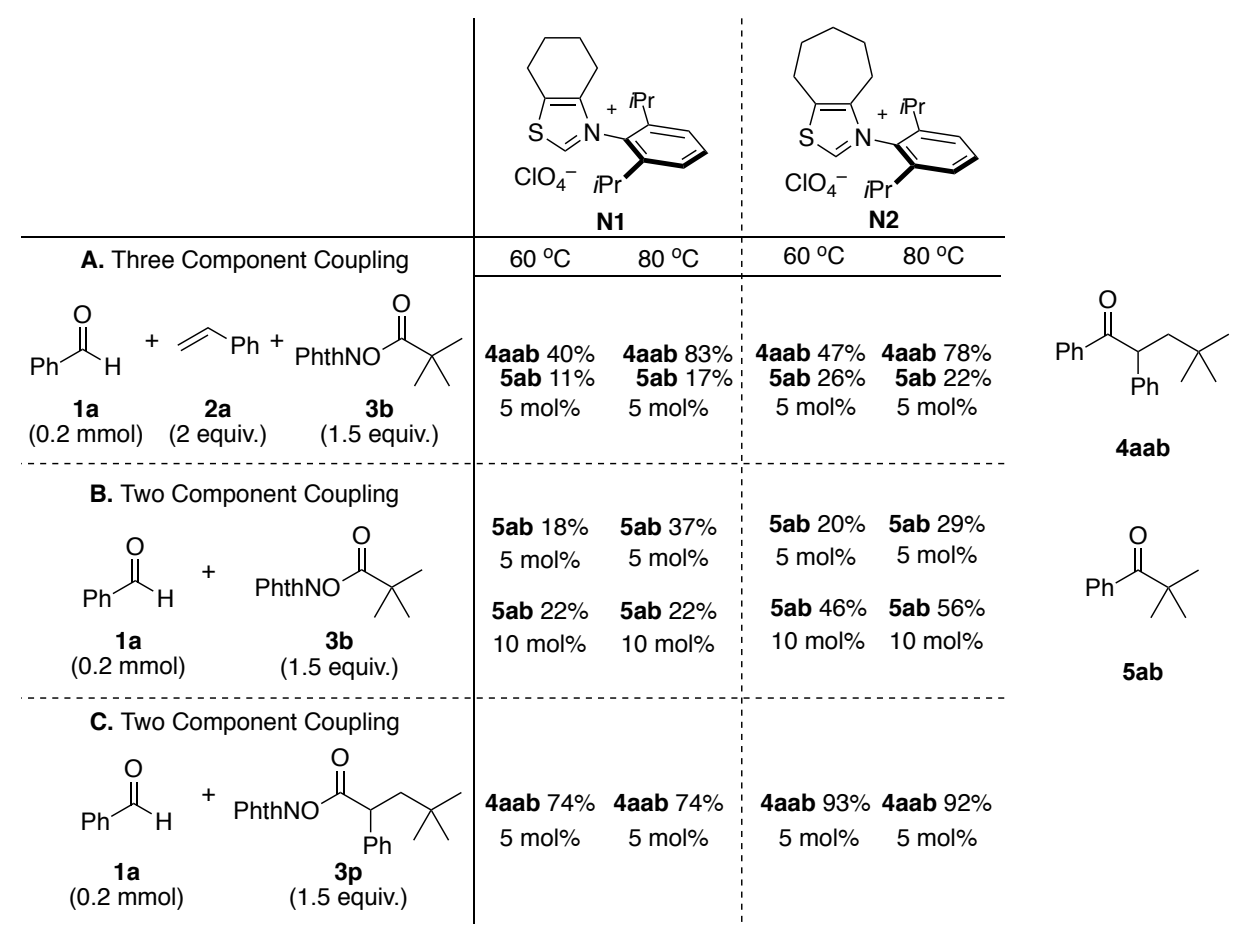

Figure S1. Two- or three-component coupling using N1 and N2

To understand the effect of NHC catalyst on the three-component coupling, we performed the several experiments using N1 and N2 (Figure S1). Based on the results shown in Figure S1A and S1C, both catalysts were found to be effective in the coupling with in situ generated benzyl radical species. However, when the reactions between aldehyde $\mathbf{1 a}$ and redox active ester $\mathbf{3 b}$ were conducted, the sixmembered N1 was less effective than the seven-membered N2 in terms of product yield of 5ab (Figure S1C). This might be due to the slow radical-radical coupling of the N1-derived ketyl radical and the tertiary alkyl radical. This is consistent with the result of Figure S1A that N1 has higher chemoselectivity than $\mathbf{N} 2$ (4aab/5ab) in the three-component coupling. More detailed mechanistic studies by theoretical calculations are underway.

\section{- Preliminary Results on Asymmetric Alkylacylation}

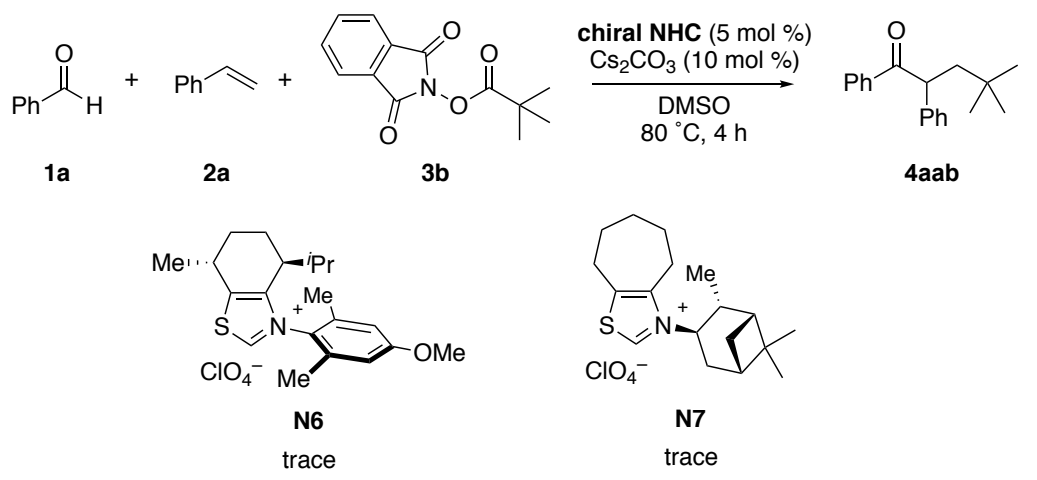

Scheme S1. Trials to asymmetric alkylacylation using chiral NHC catalyst (N6 or N7) 


\section{- References匹}

(1) Qin, T.; Cornella, J.; Li, C.; Malins, L. R.; Edwards, J. T.; Kawamura, S.; Maxwell, B. D.; Eastgate, M. D.; Baran, P. S. Science 2016, 352, 801.

(2) Piel, I.; Pawelczyk, M. D.; Hirano, K.; Fröhlich, R.; Glorius, F.; Eur. J. Org. Chem. 2011, 5475.

(3) Pan, C,; Ni, Q,; Fu, Y.; Yu, J.-T.; J. Org. Chem. 2017, 82, 7683.

(4) Zhao, J.; Fang, H.; Song, R.; Zhou, J.; Han, J.; Pan, Y.; Chem. Commun., 2015, 51, 599. 


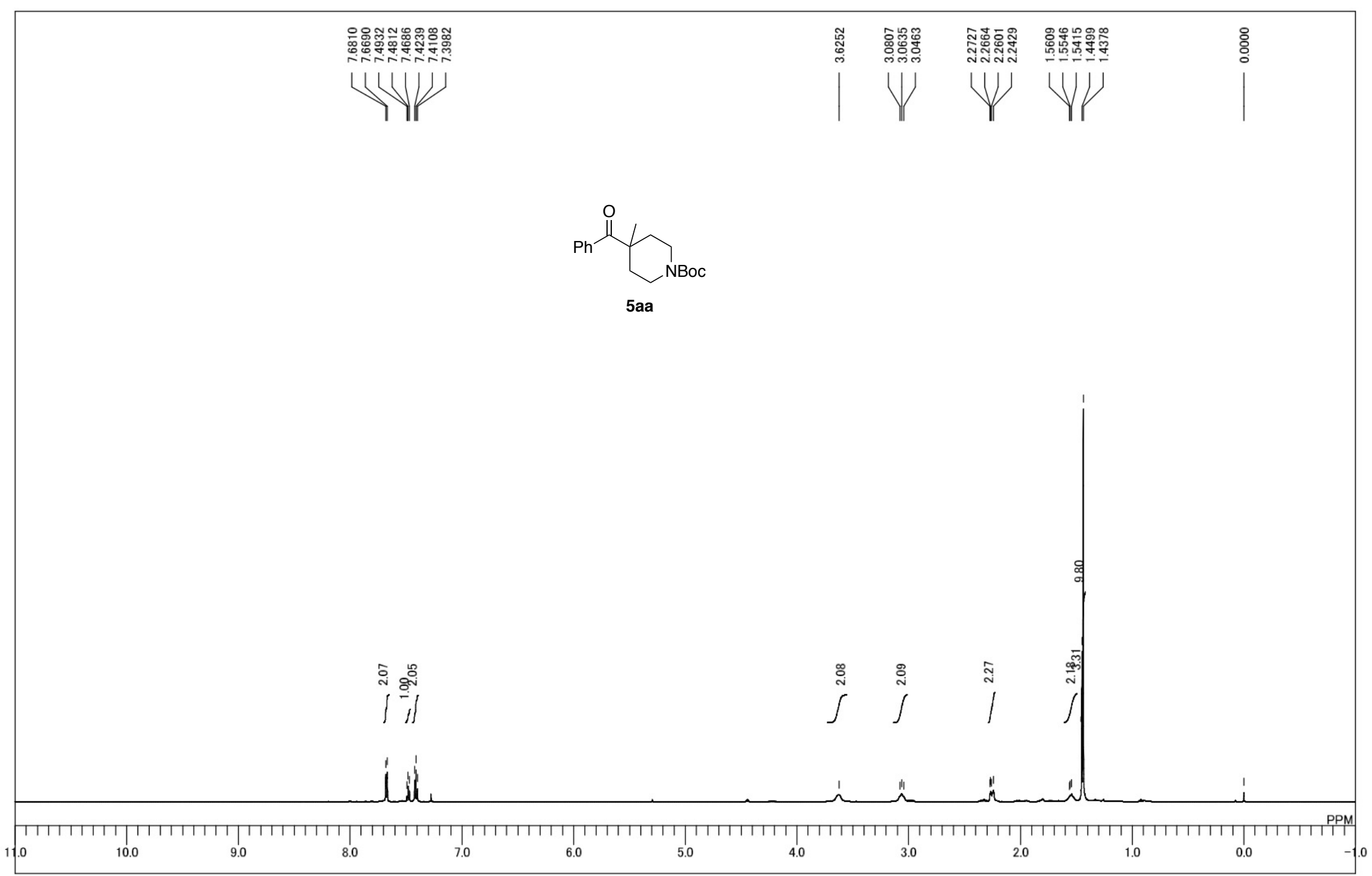

${ }^{1} \mathrm{H}$ NMR spectrum of $\mathbf{5 a a}$ 


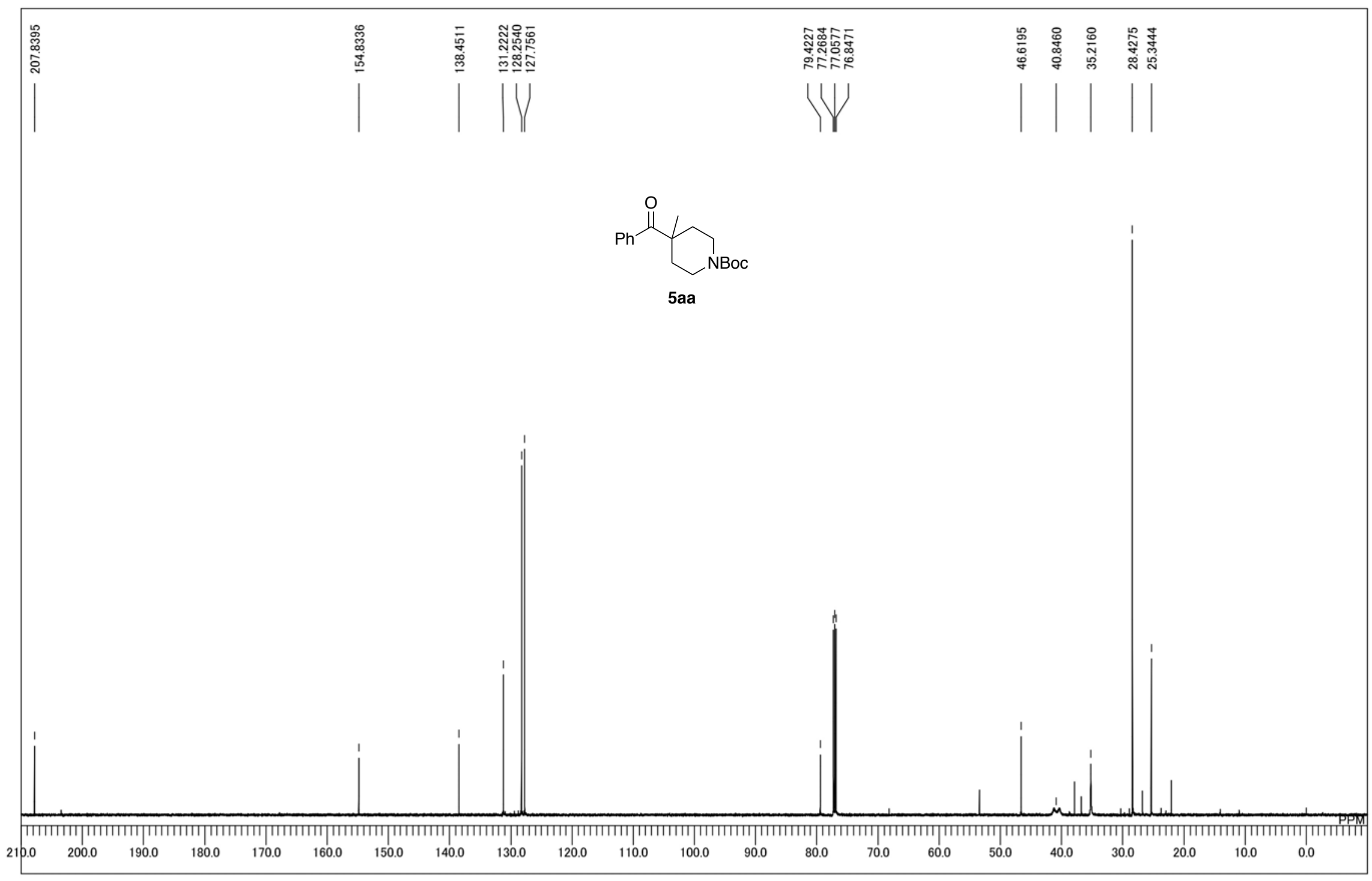

${ }^{13} \mathrm{C}$ NMR spectrum of $5 \mathbf{a a}$ 


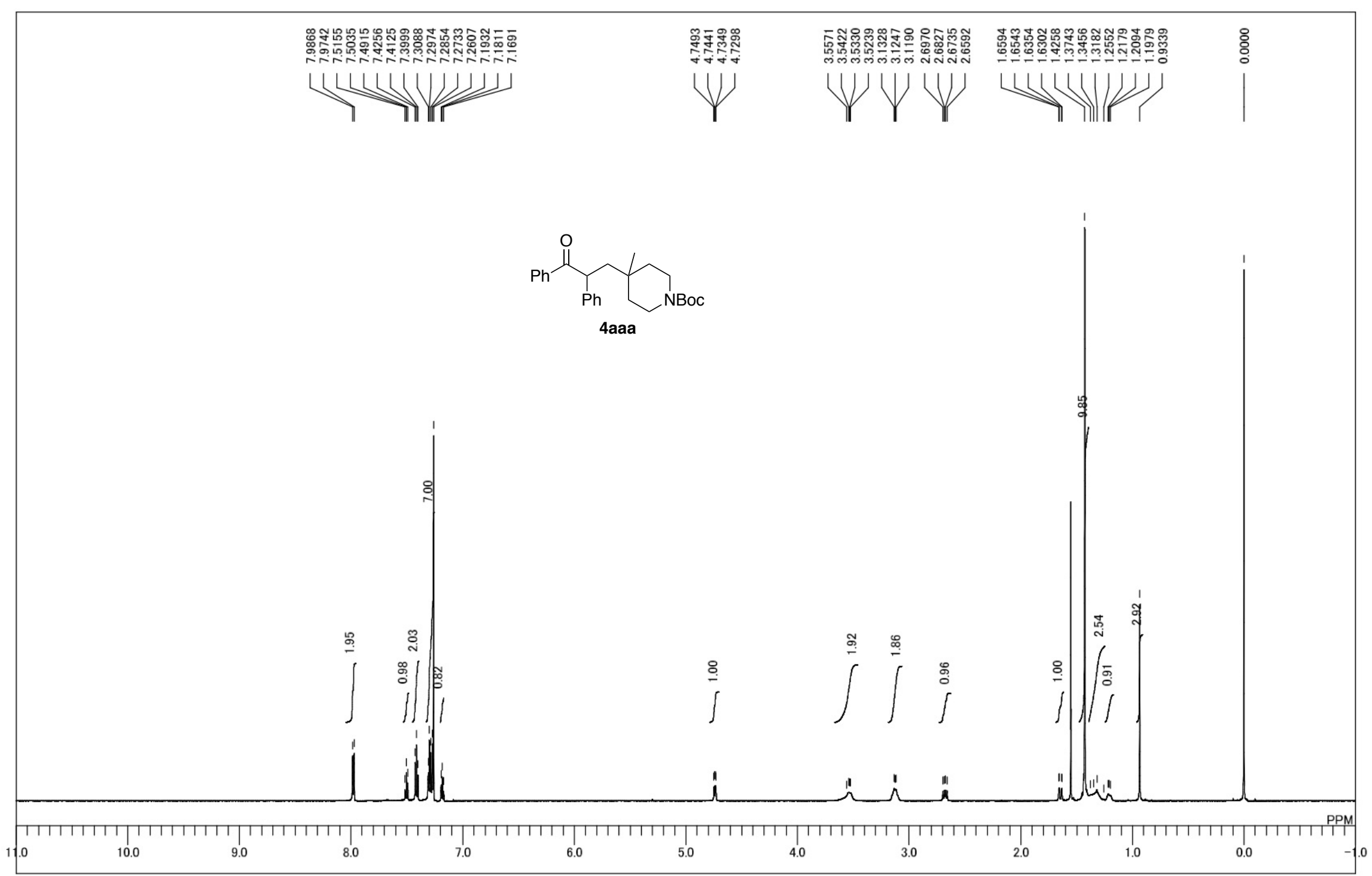

${ }^{1} \mathrm{H}$ NMR spectrum of 4 aaa 


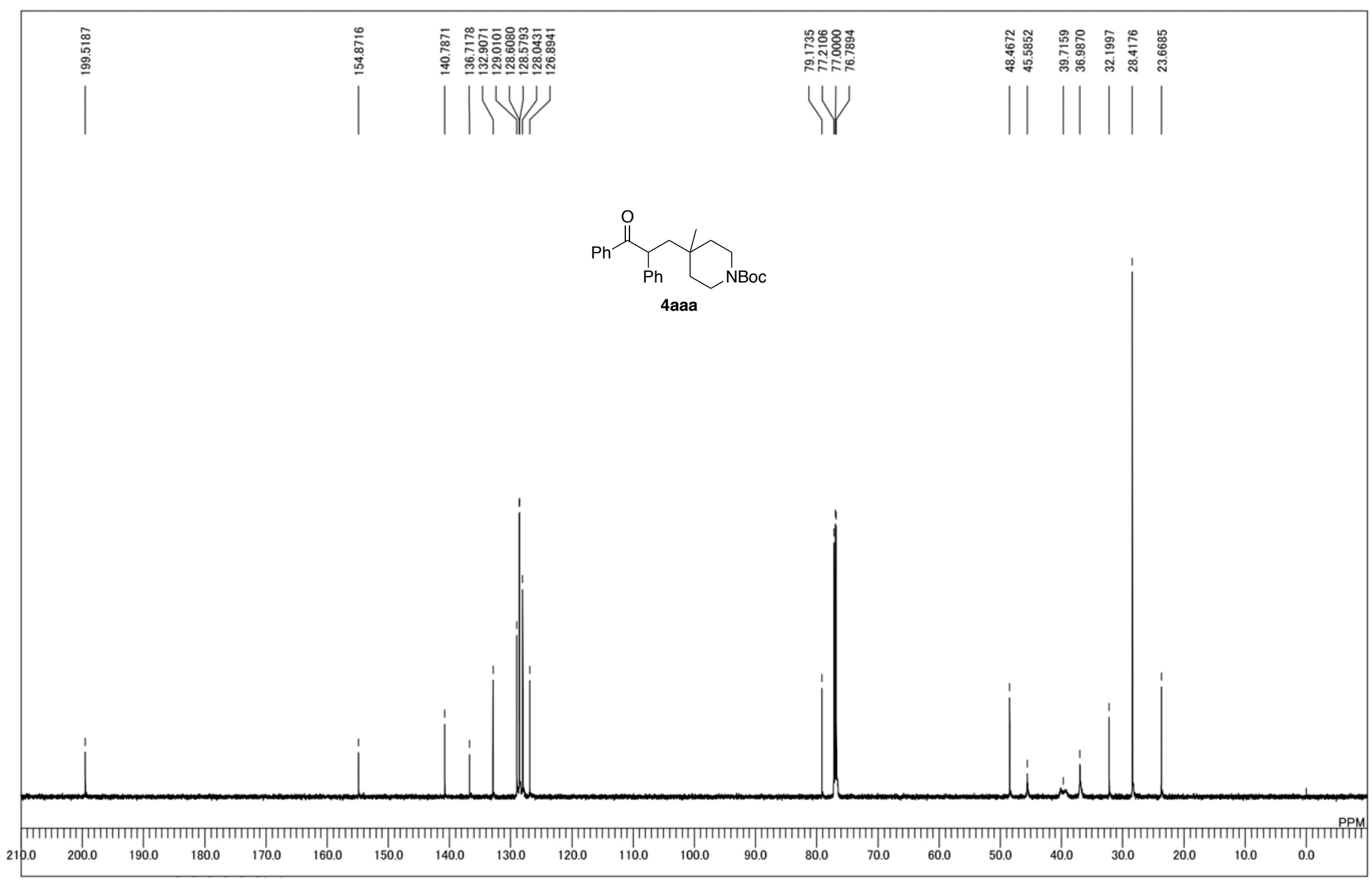

${ }^{13} \mathrm{C}$ NMR spectrum of 4 aaa 


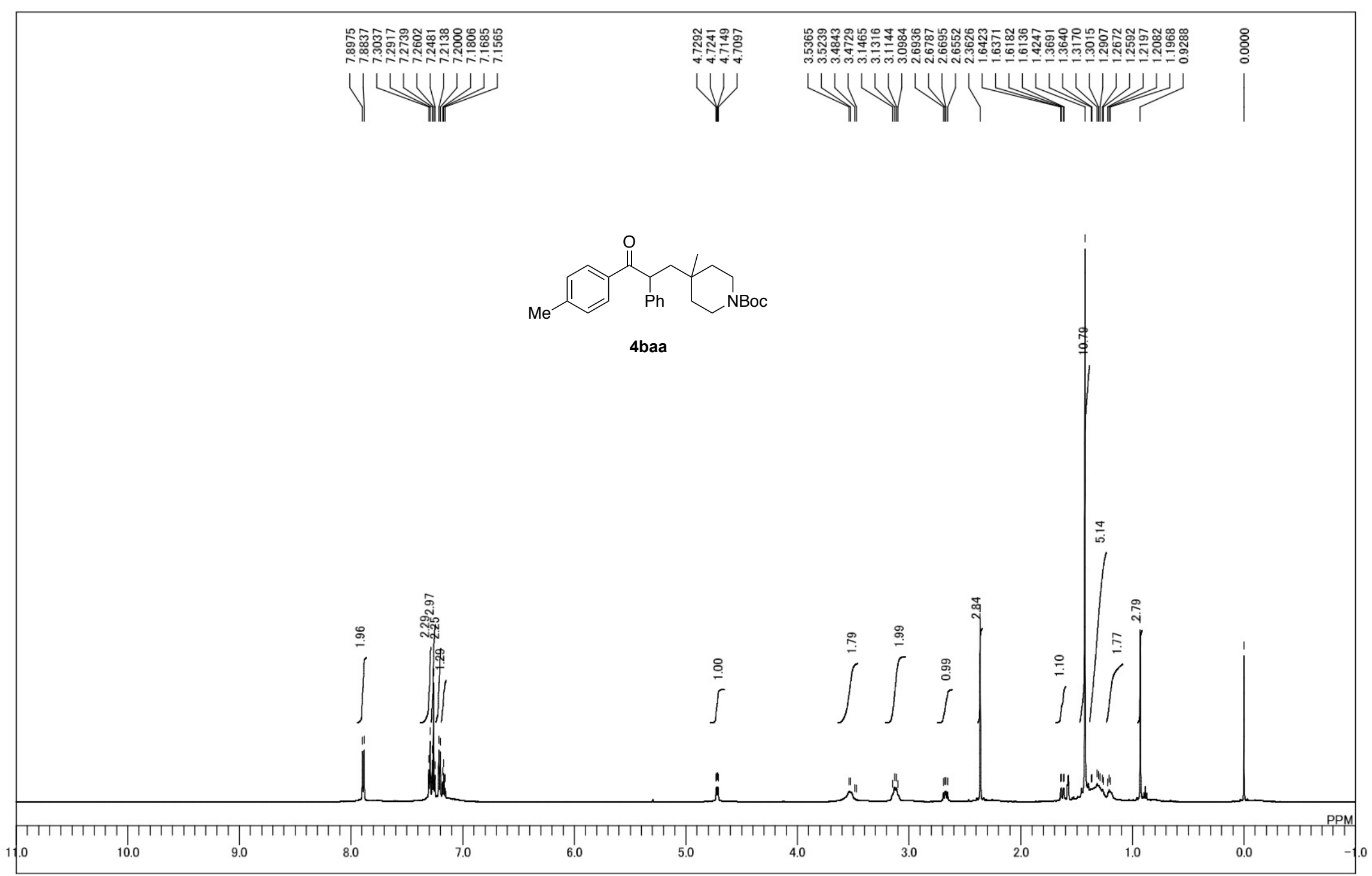

${ }^{1} \mathrm{H}$ NMR spectrum of $\mathbf{4 b a a}$ 


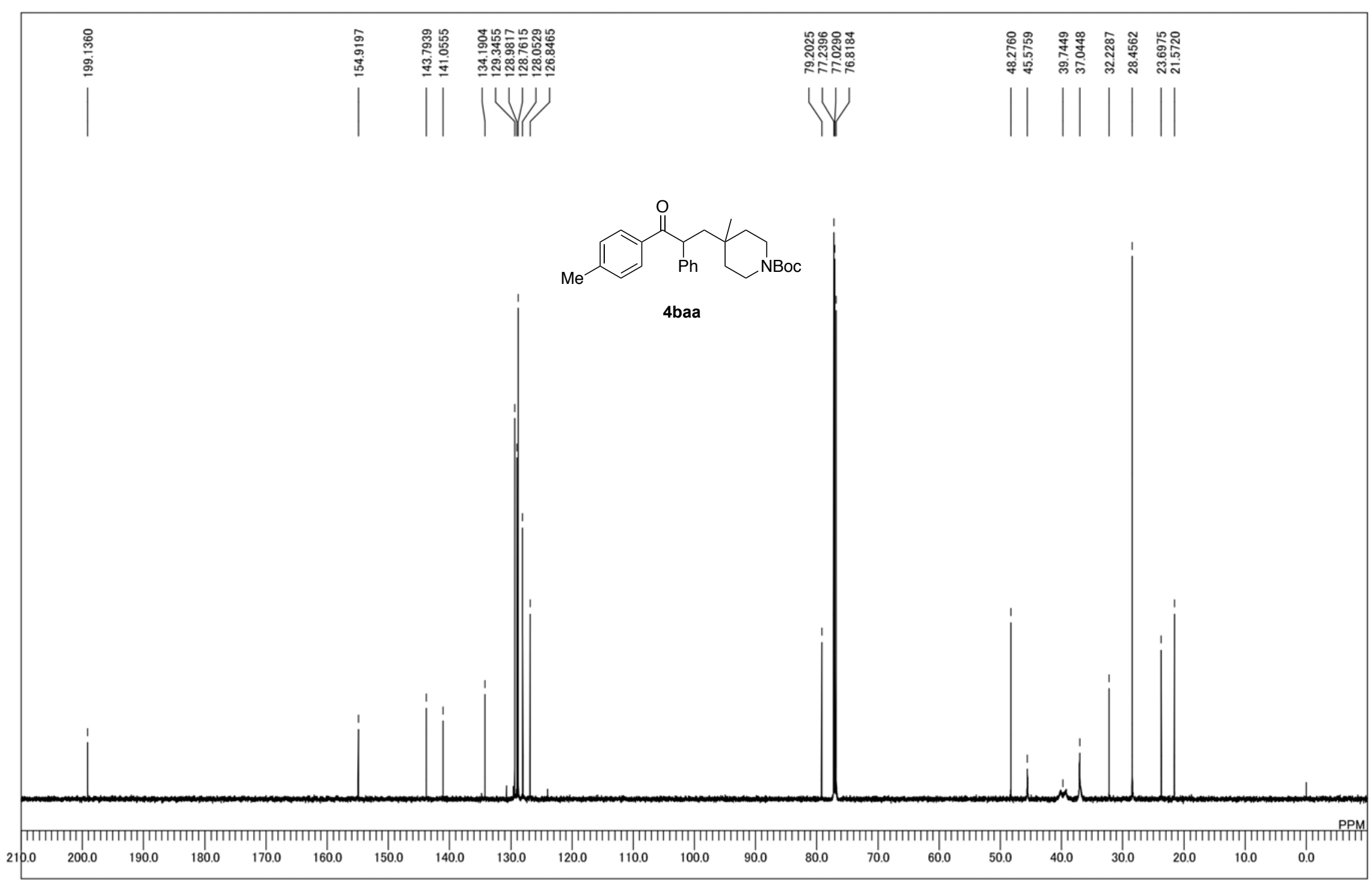

${ }^{13} \mathrm{C}$ NMR spectrum of $4 \mathrm{baa}$ 


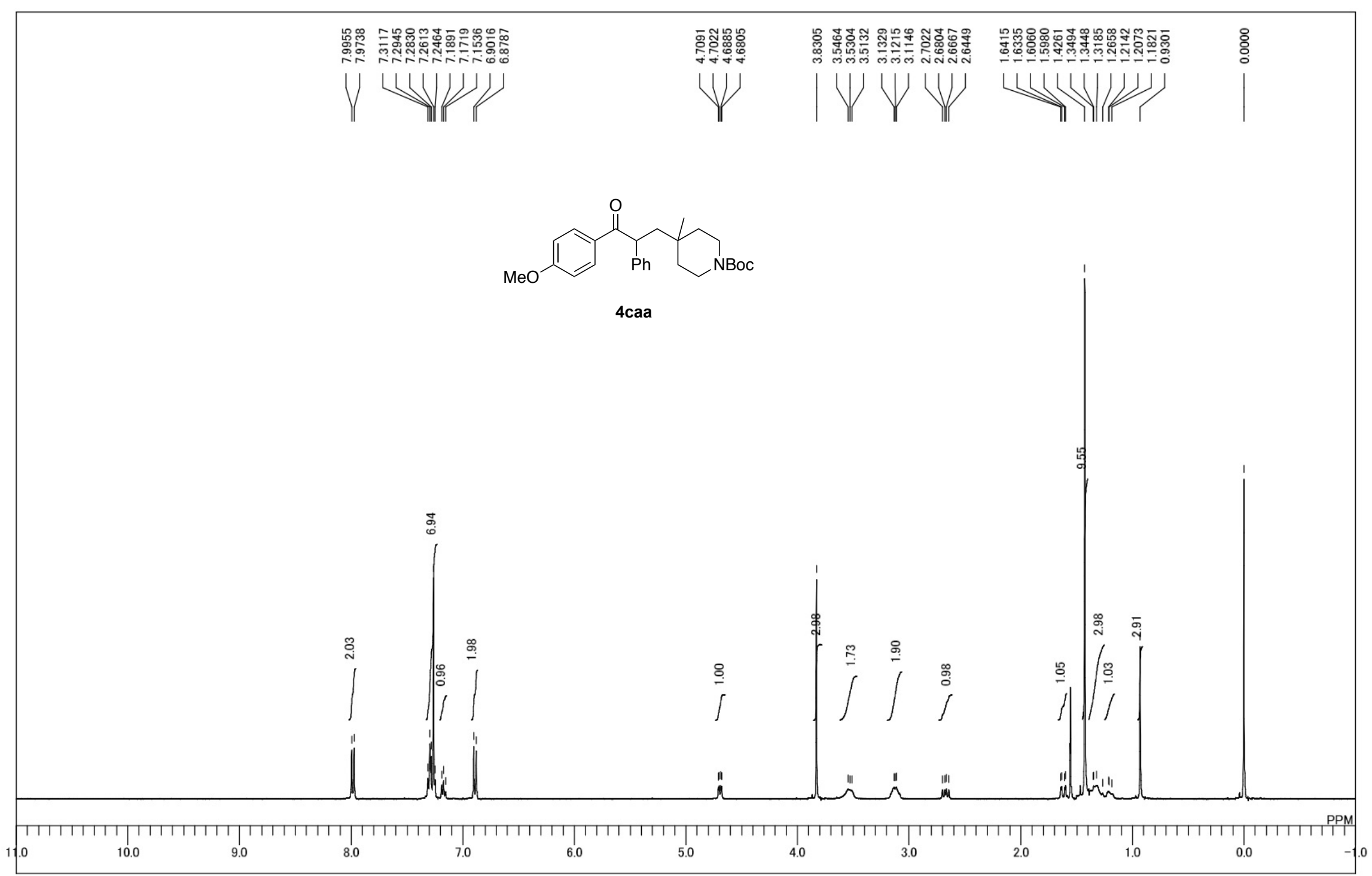

${ }^{1} \mathrm{H}$ NMR spectrum of $\mathbf{4 c a a}$ 


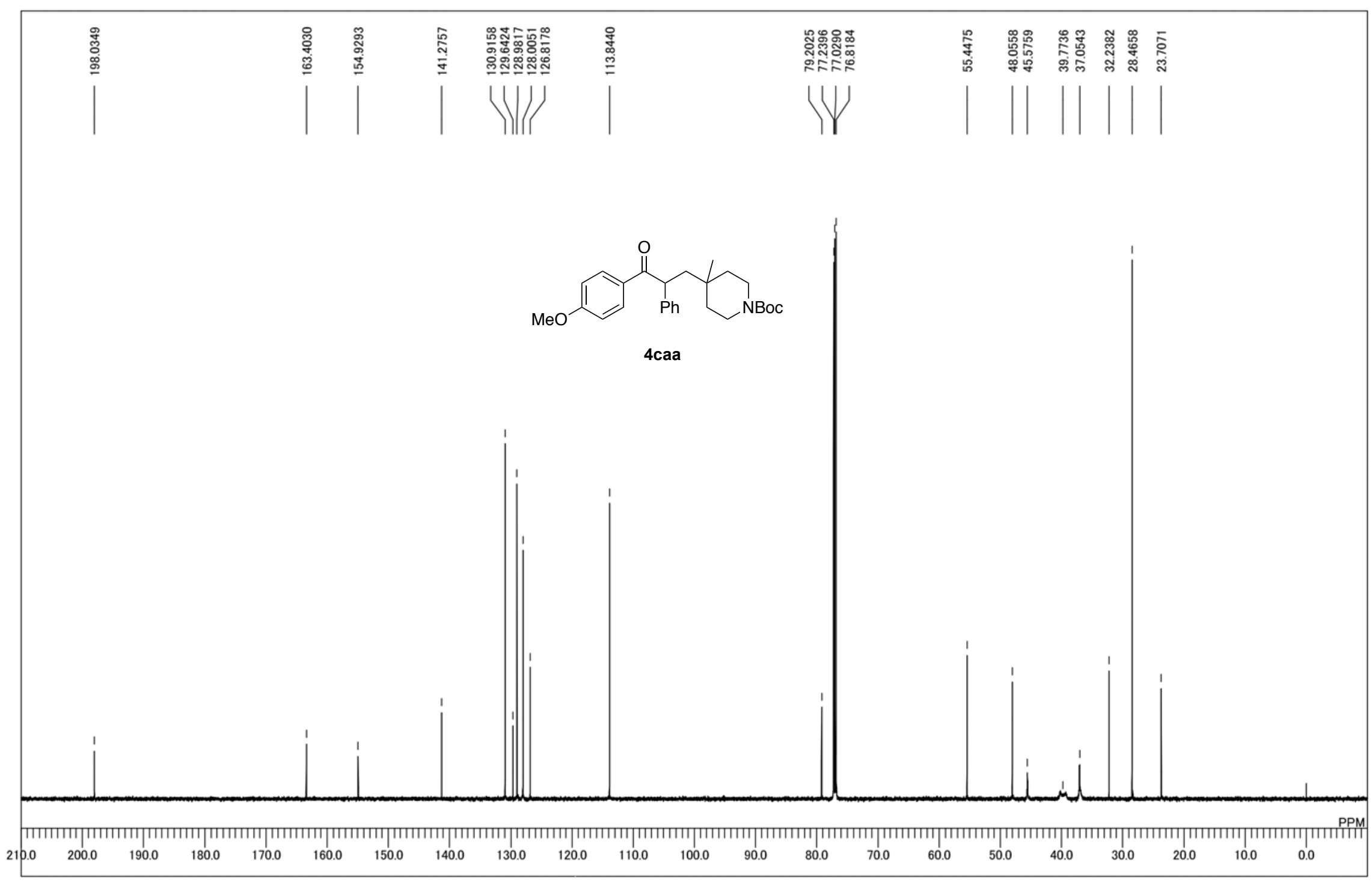

${ }^{13} \mathrm{C}$ NMR spectrum of 4caa 


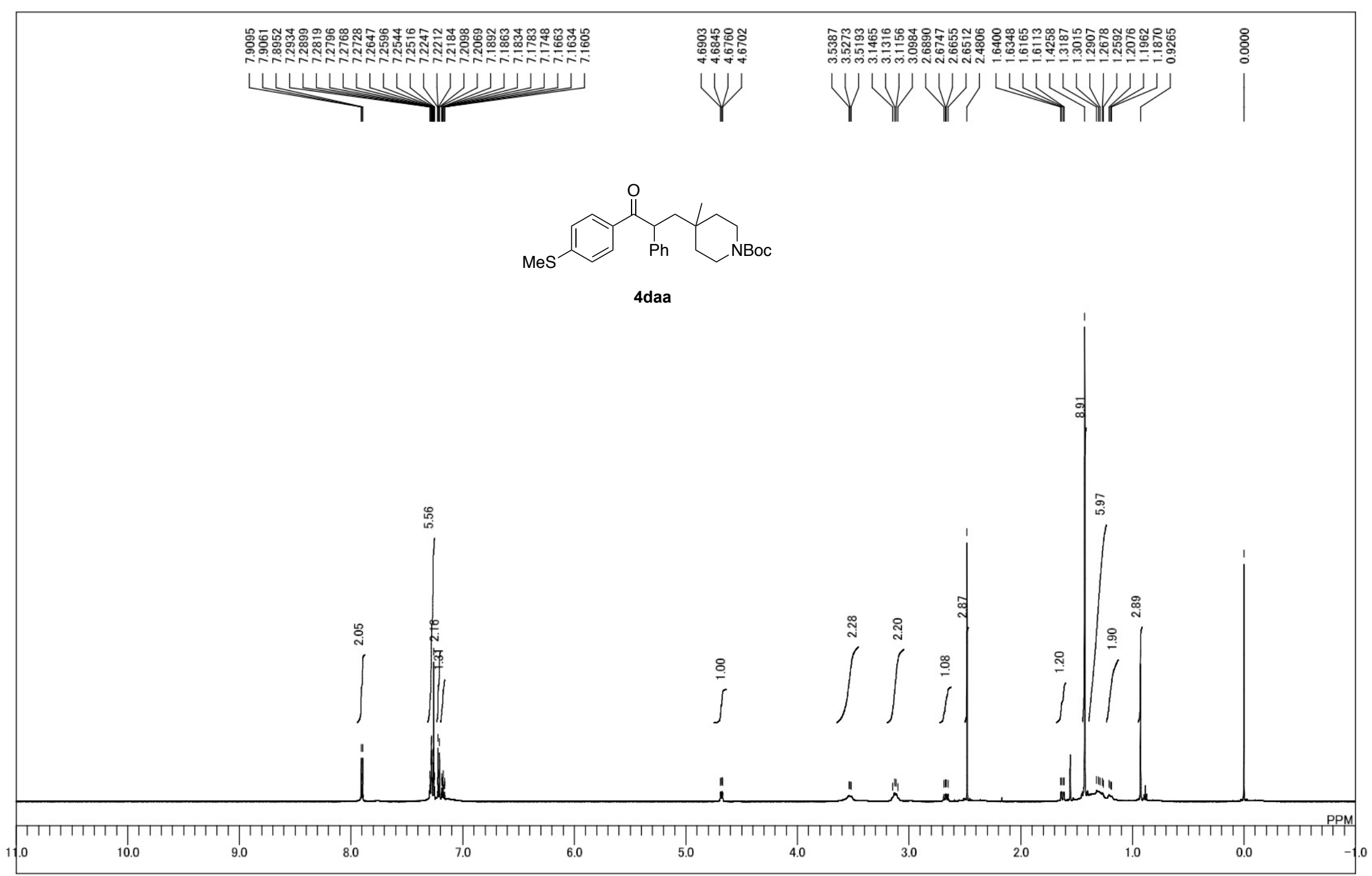

${ }^{1} \mathrm{H}$ NMR spectrum of $\mathbf{4 d a a}$ 


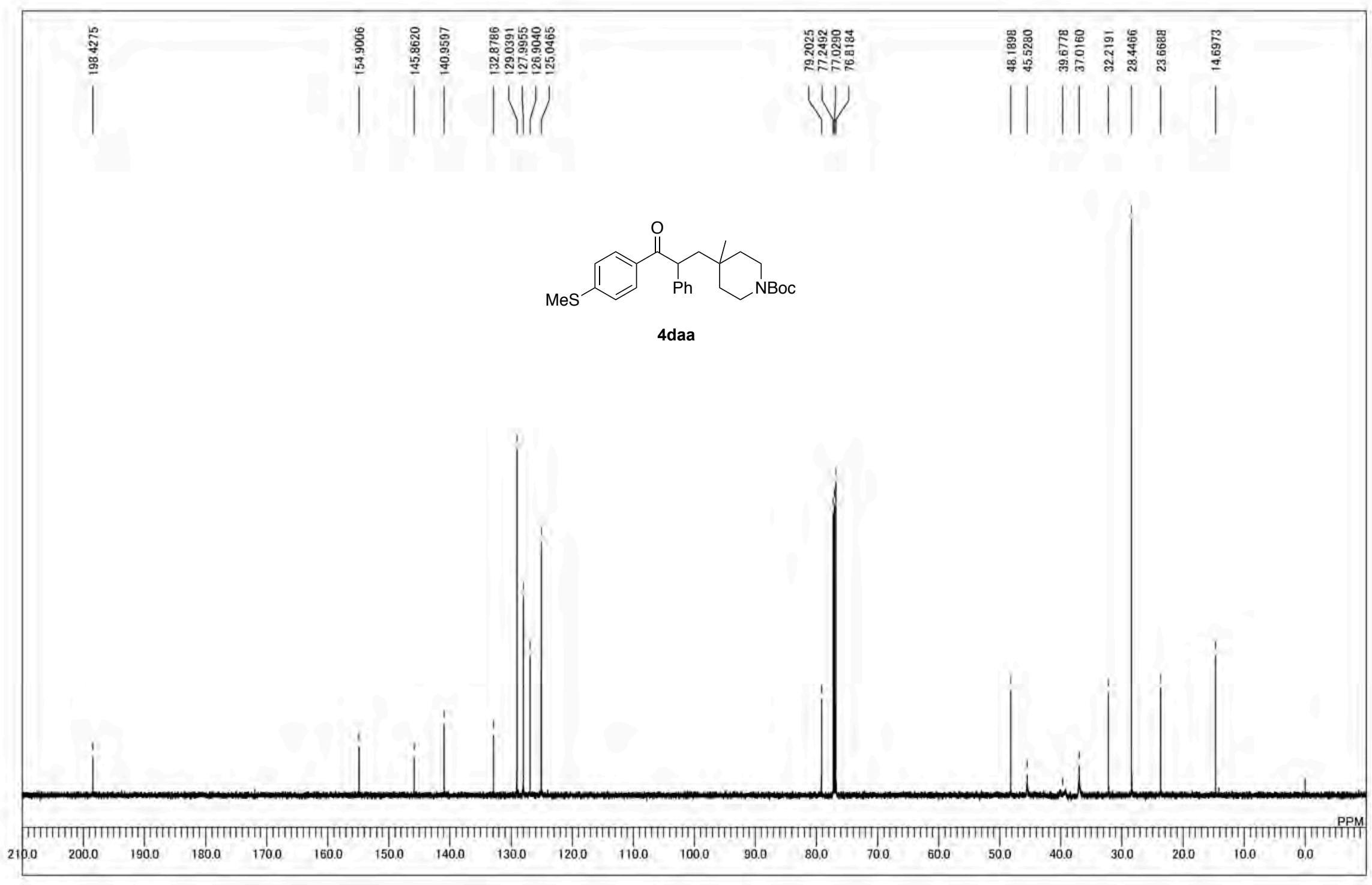

${ }^{13} \mathrm{C}$ NMR spectrum of $\mathbf{4 d a a}$ 


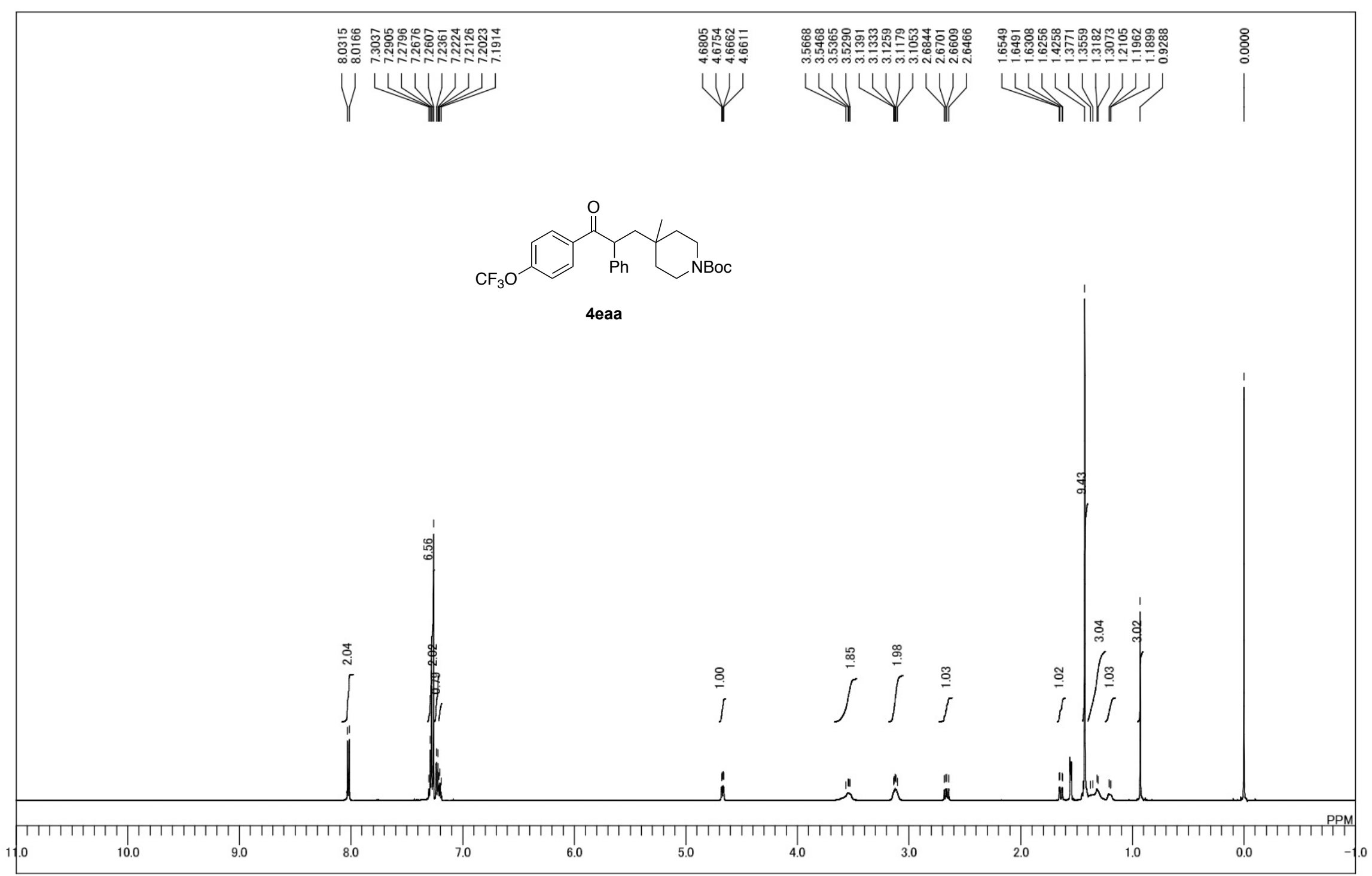

${ }^{1} \mathrm{H}$ NMR spectrum of $4 \mathbf{e a a}$ 


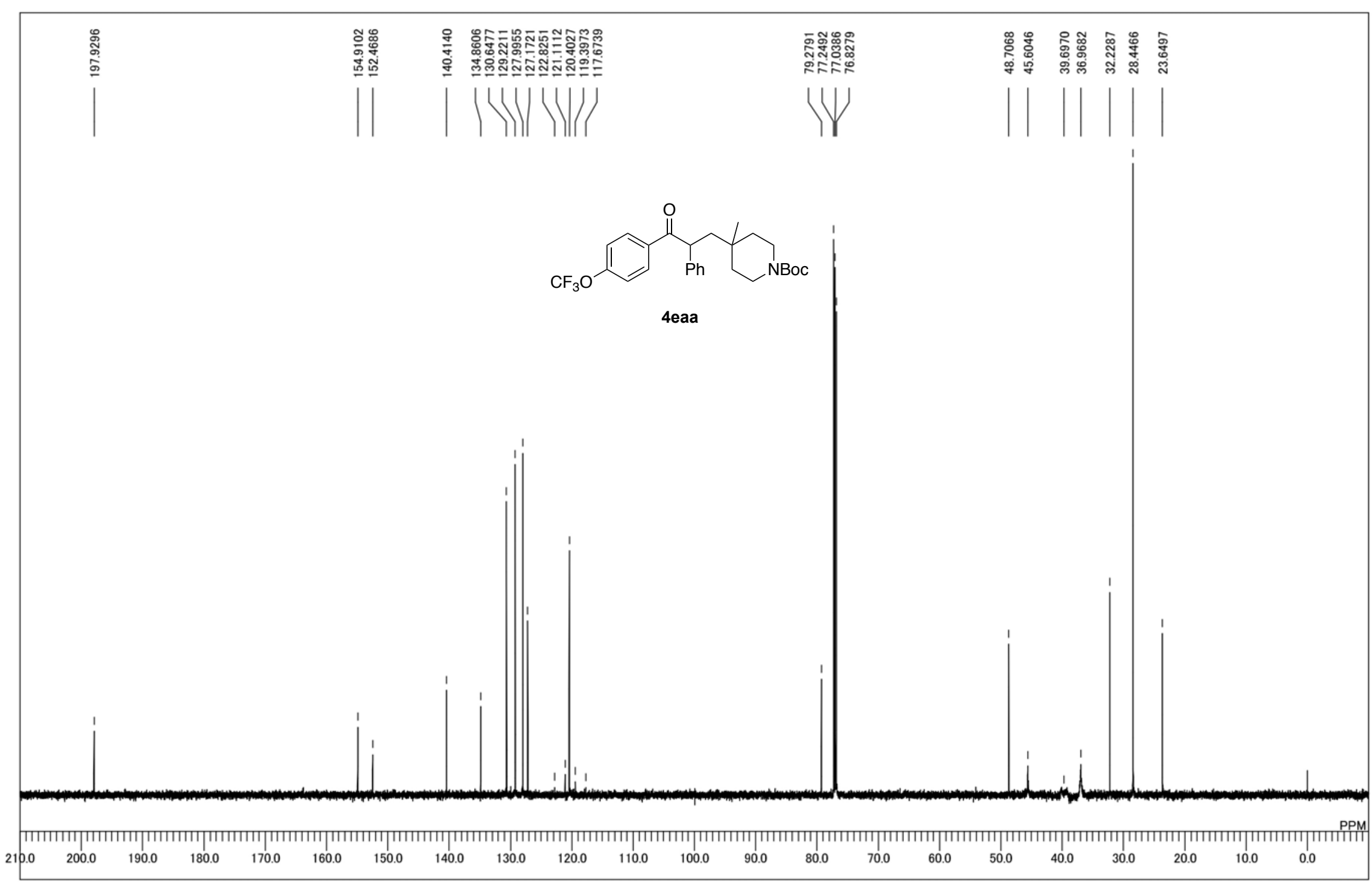

${ }^{13} \mathrm{C}$ NMR spectrum of 4eaa 


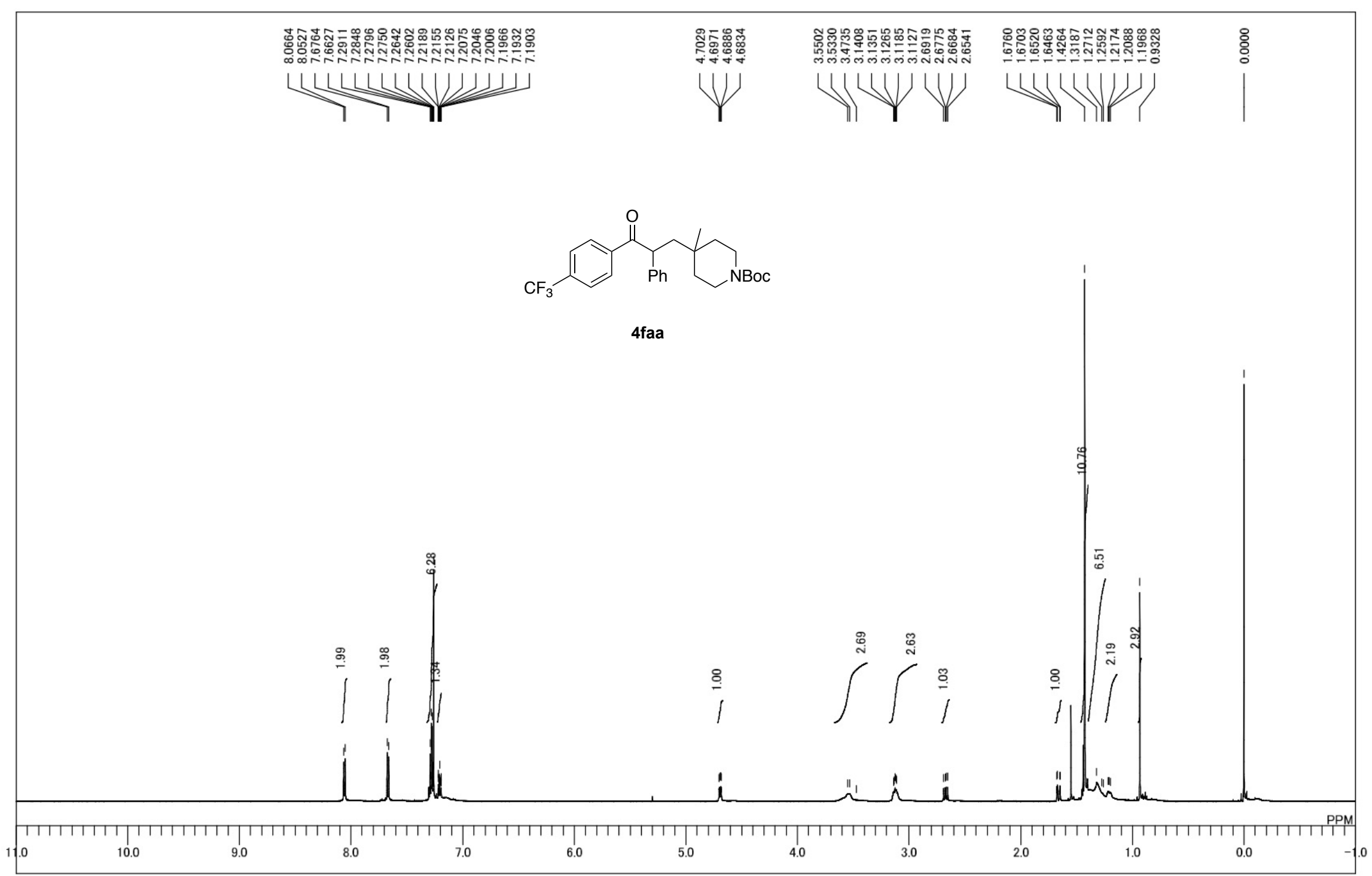

${ }^{1} \mathrm{H}$ NMR spectrum of $\mathbf{4} \mathbf{f a a}$ 


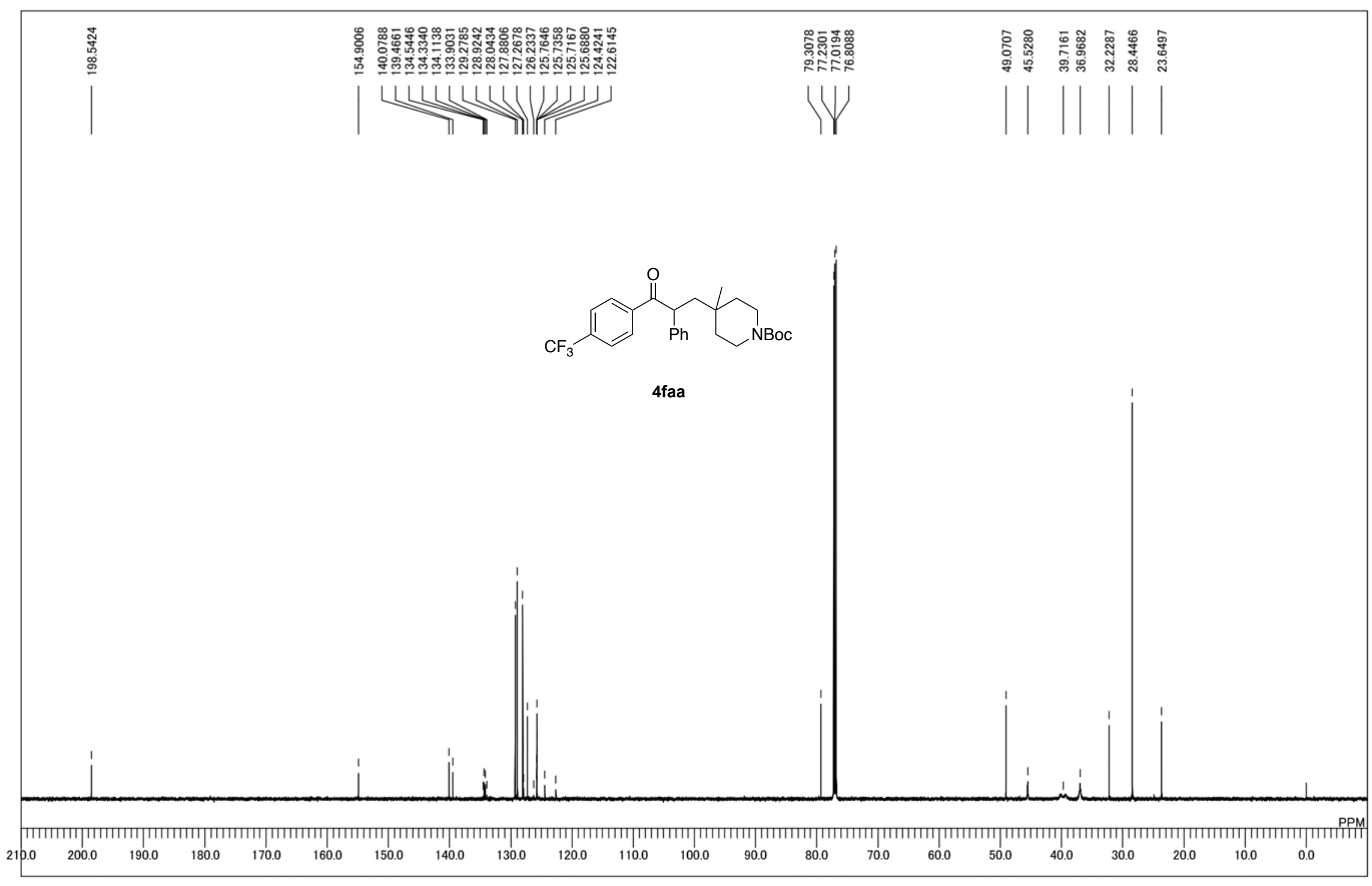

${ }^{13} \mathrm{C}$ NMR spectrum of $\mathbf{4 f a a}$ 


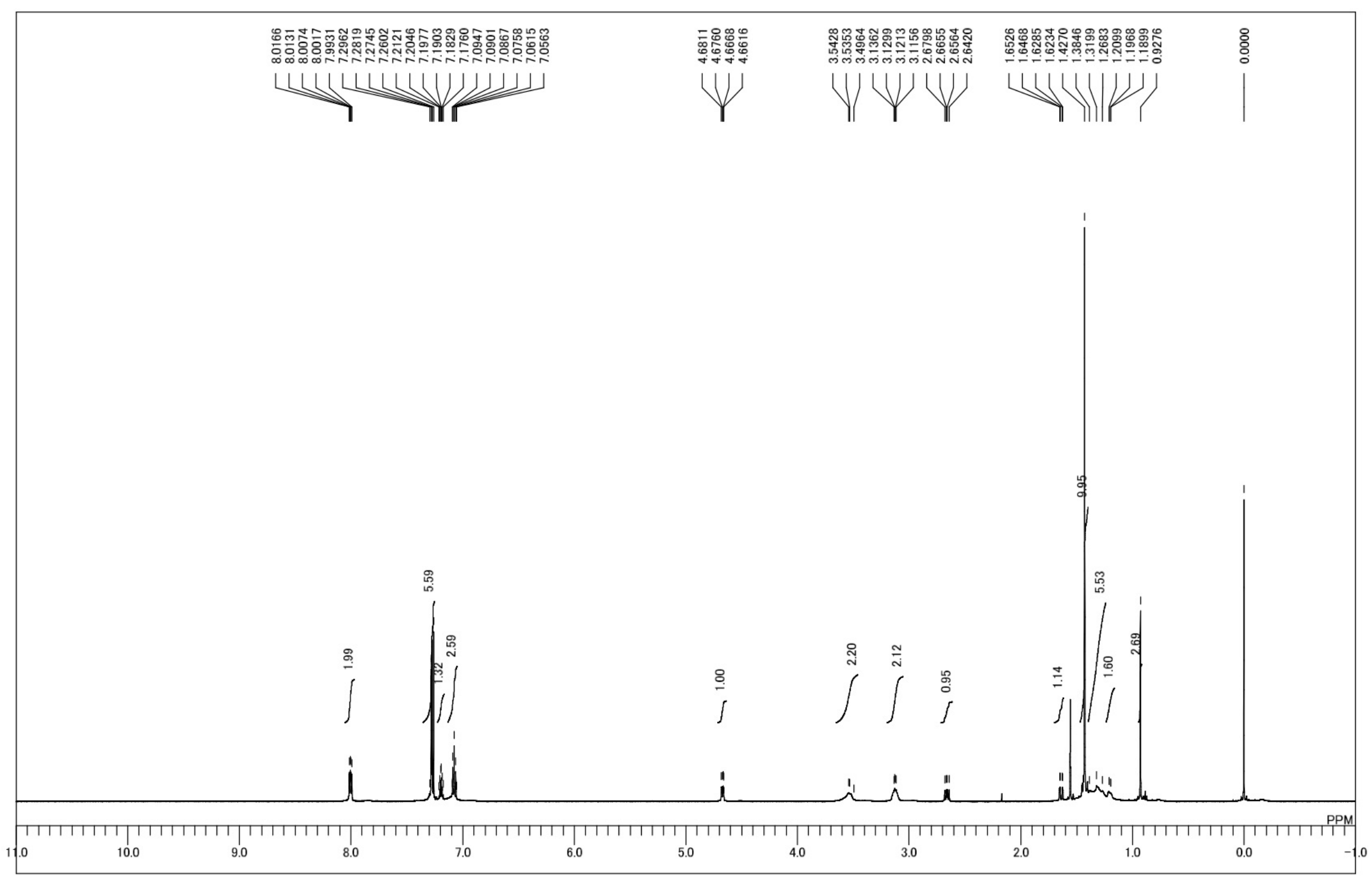

${ }^{1} \mathrm{H}$ NMR spectrum of 4gaa 


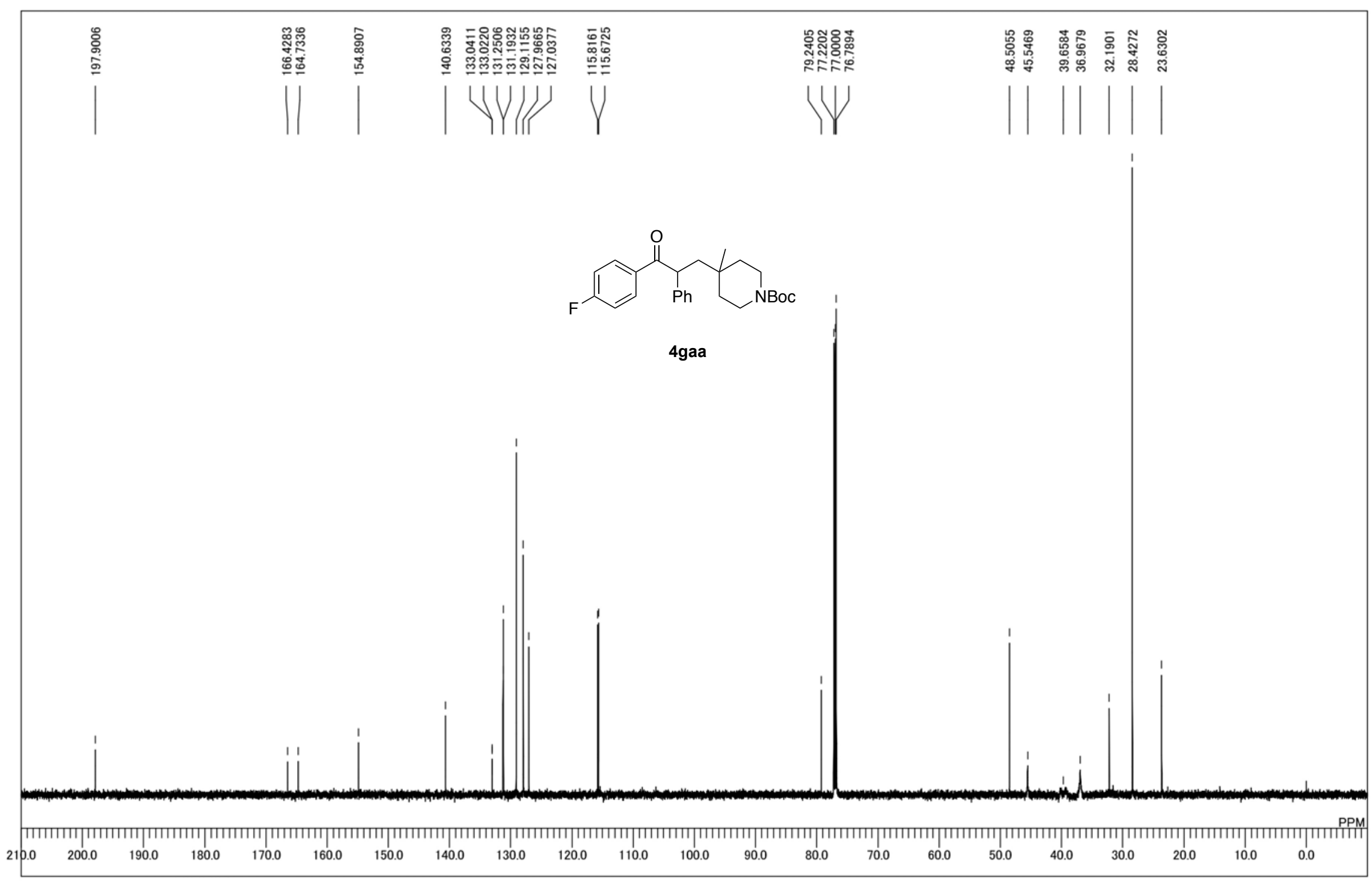

${ }^{13} \mathrm{C}$ NMR spectrum of 4 gaa 


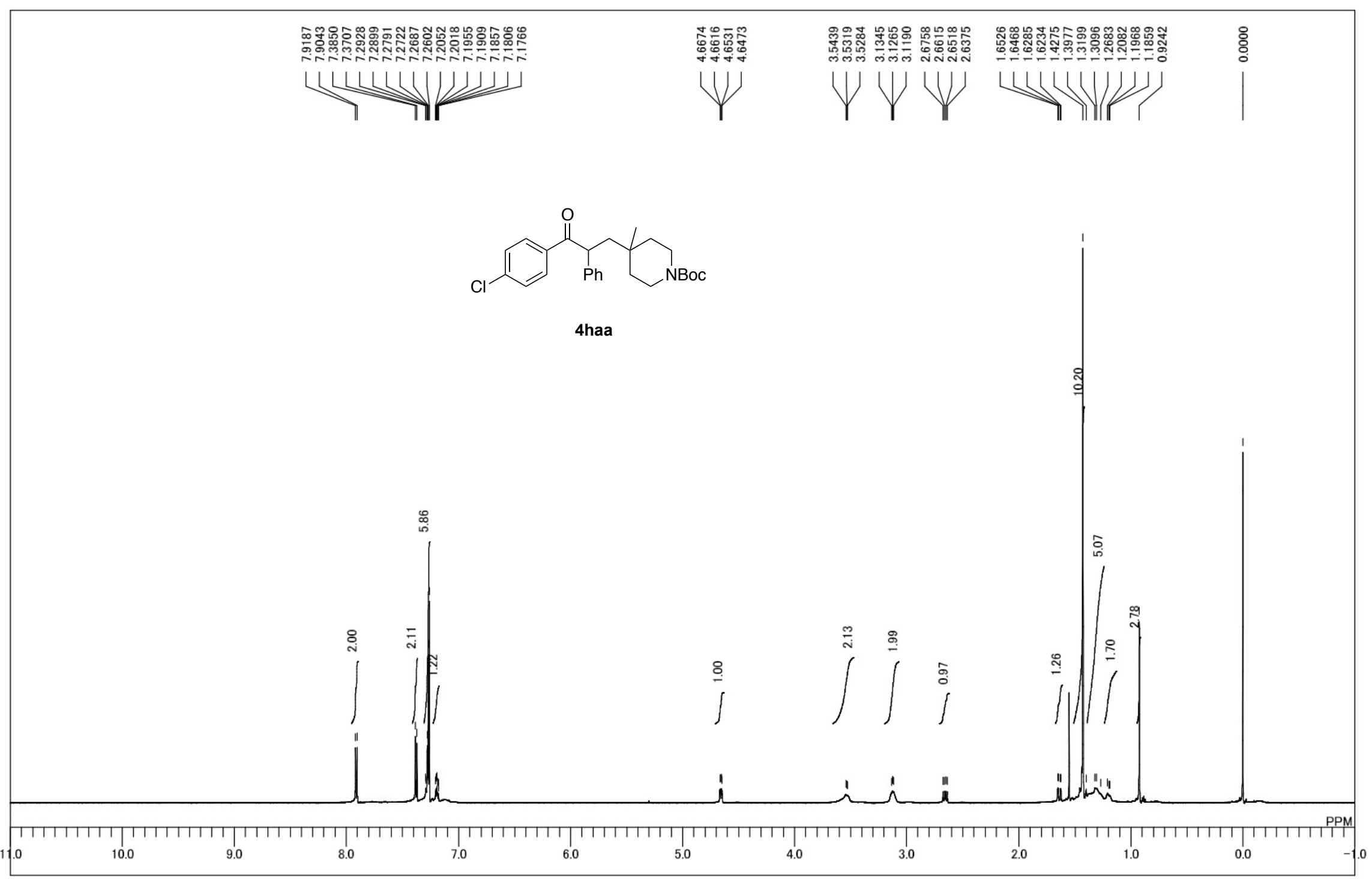

${ }^{1} \mathrm{H}$ NMR spectrum of 4 haa 


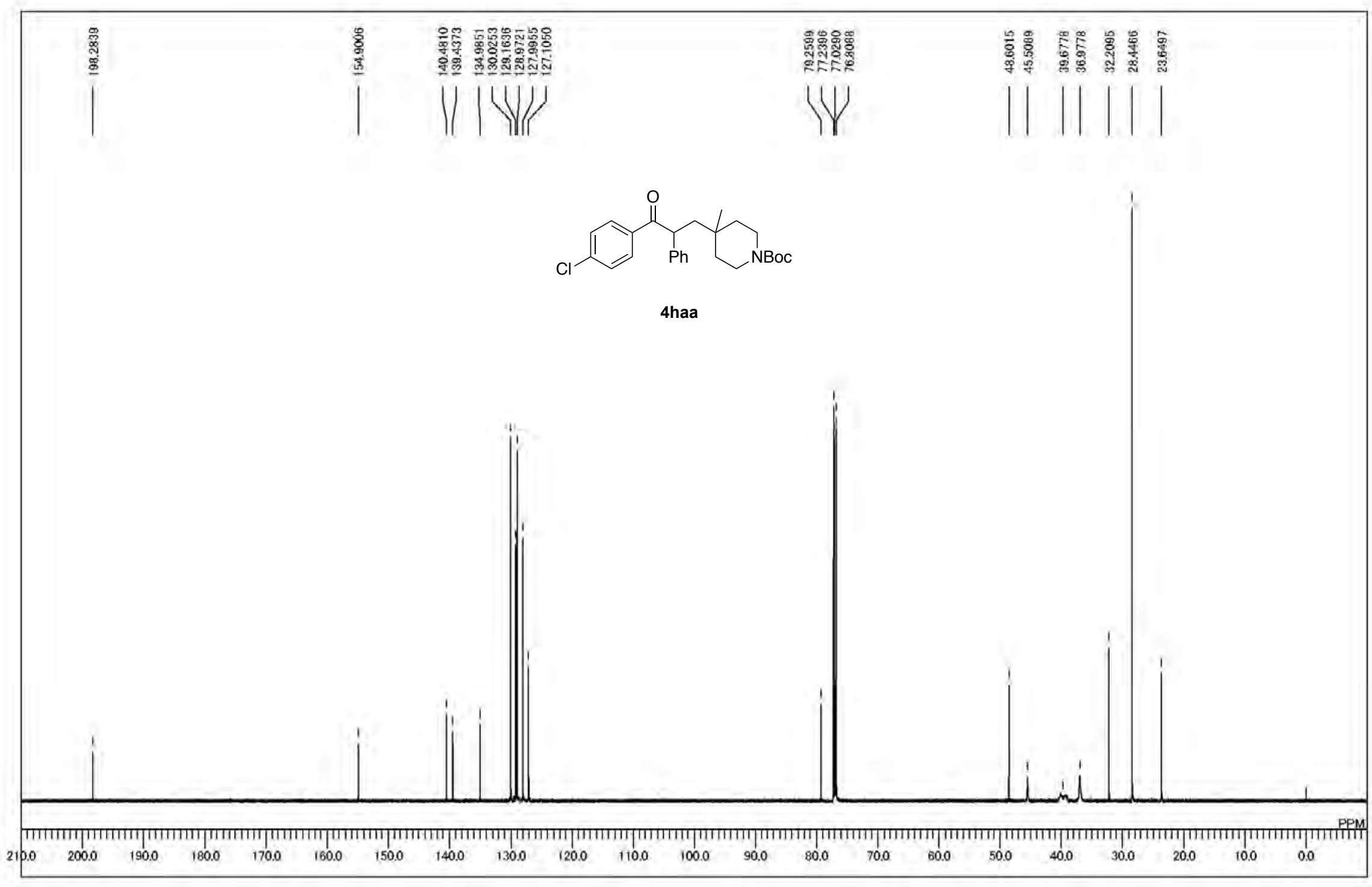

${ }^{13} \mathrm{C}$ NMR spectrum of 4 haa 


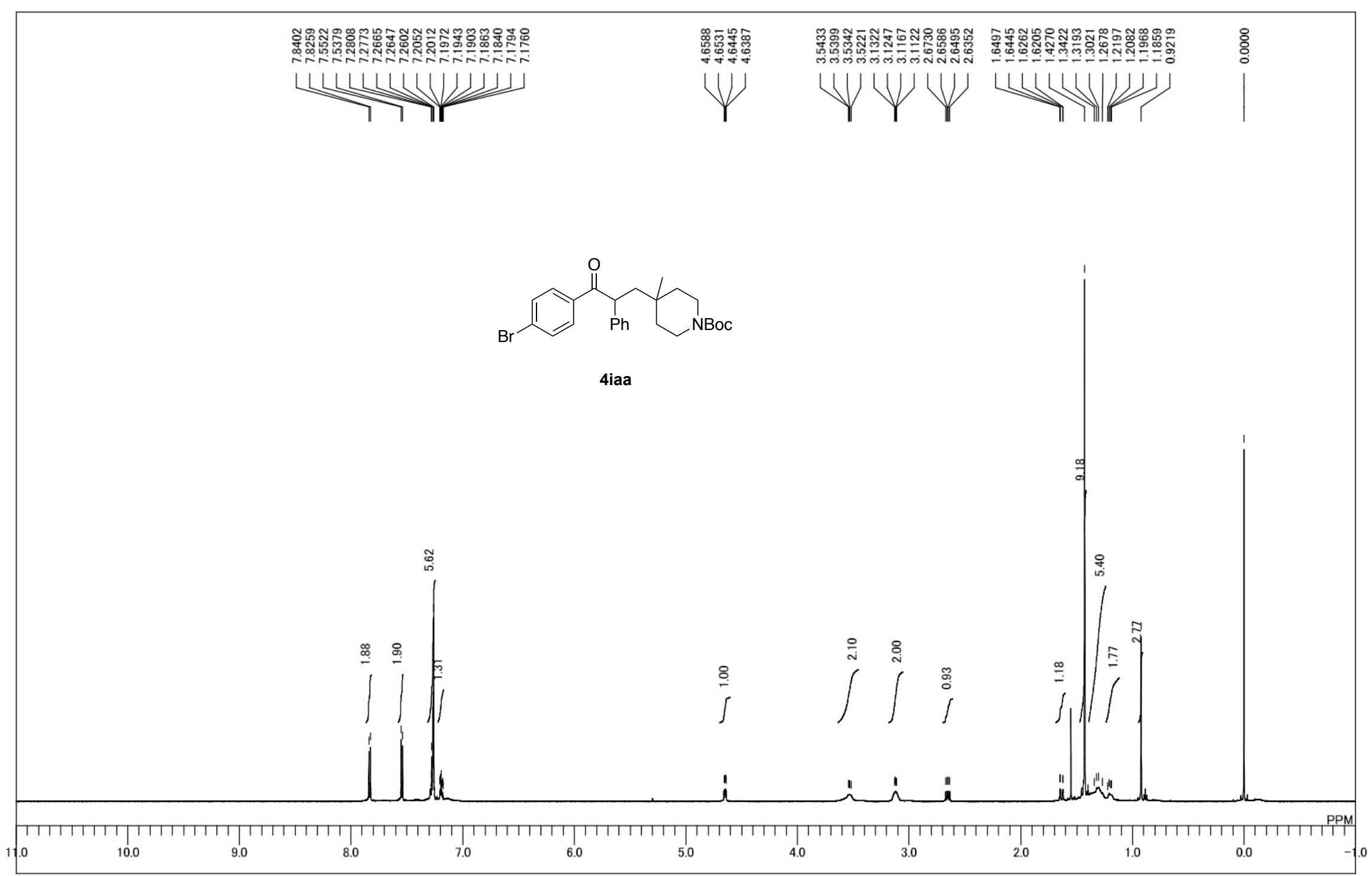

${ }^{1} \mathrm{H}$ NMR spectrum of 4iaa 


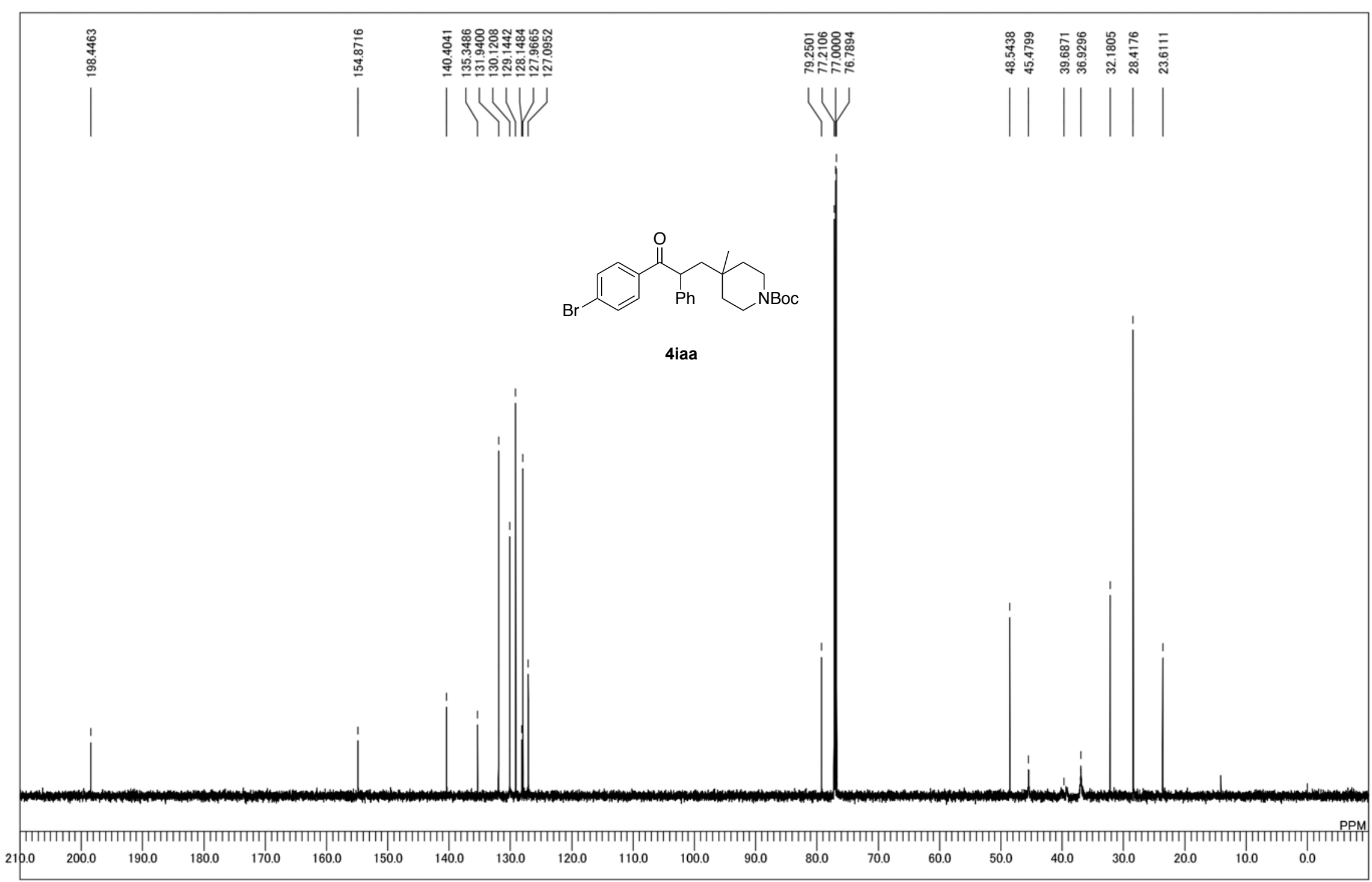

${ }^{13} \mathrm{C}$ NMR spectrum of 4iaa 


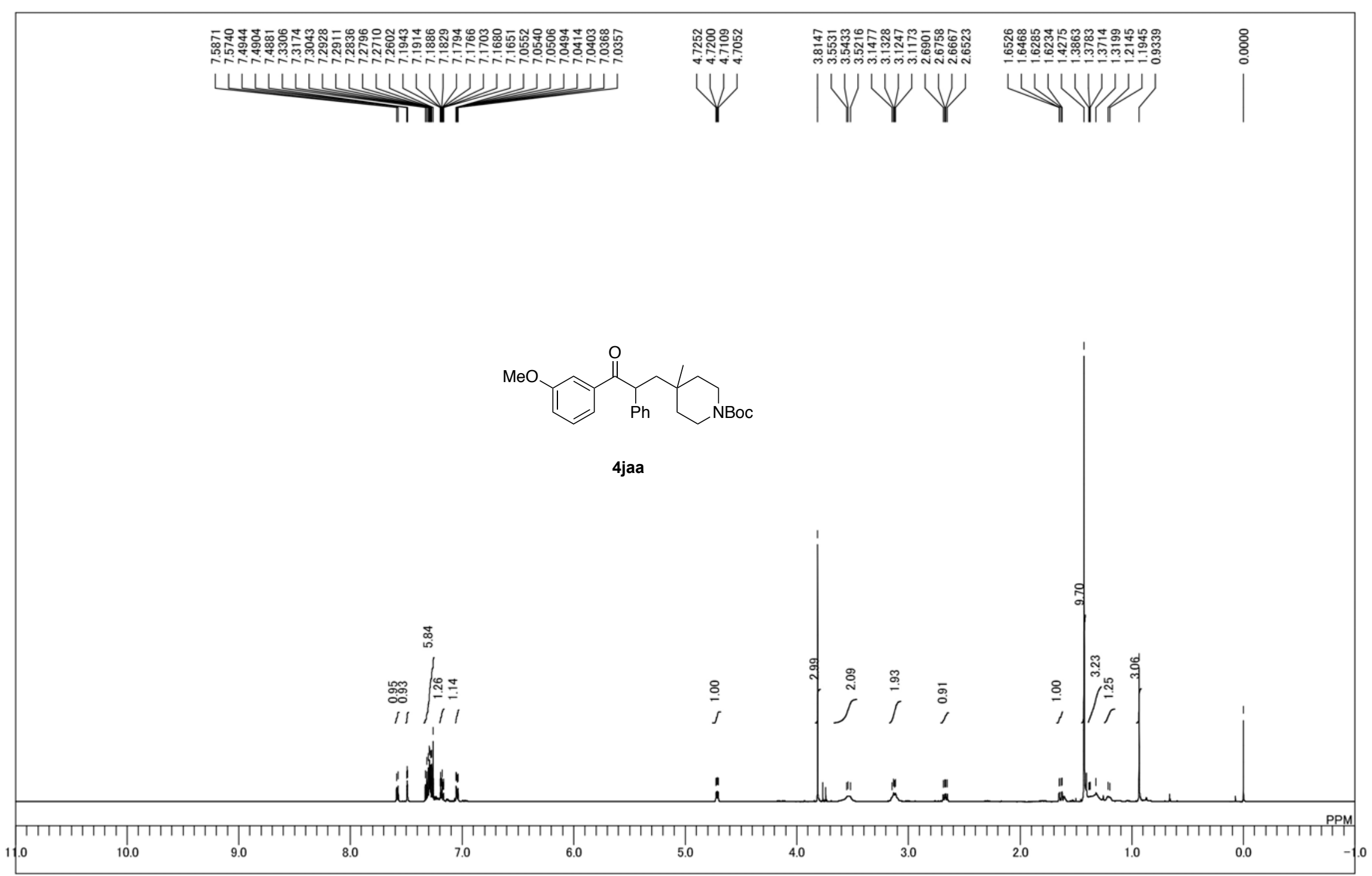

${ }^{1} \mathrm{H}$ NMR spectrum of $\mathbf{4} \mathbf{j a a}$ 


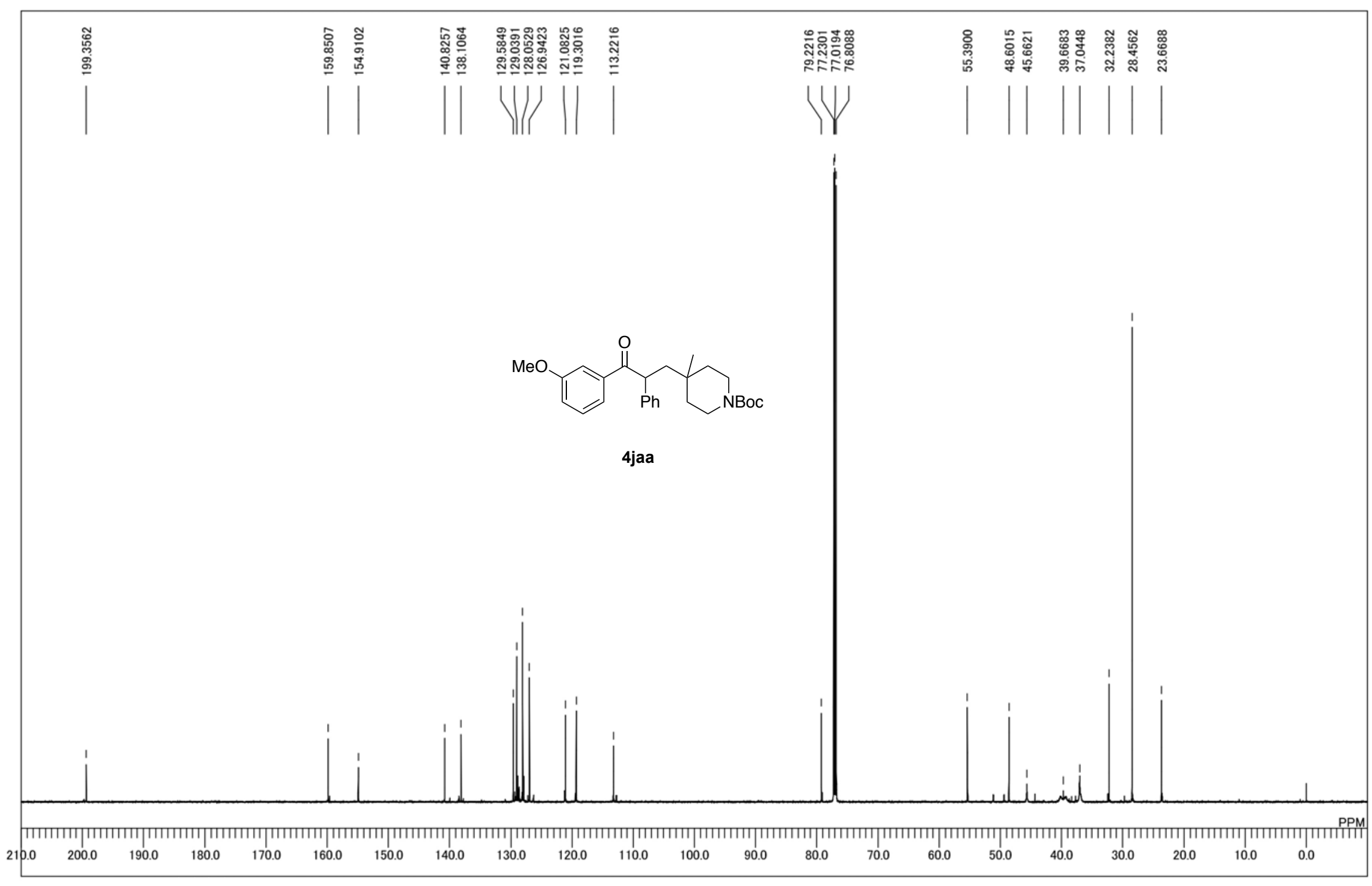

${ }^{13} \mathrm{C}$ NMR spectrum of $\mathbf{4} \mathbf{j a a}$ 


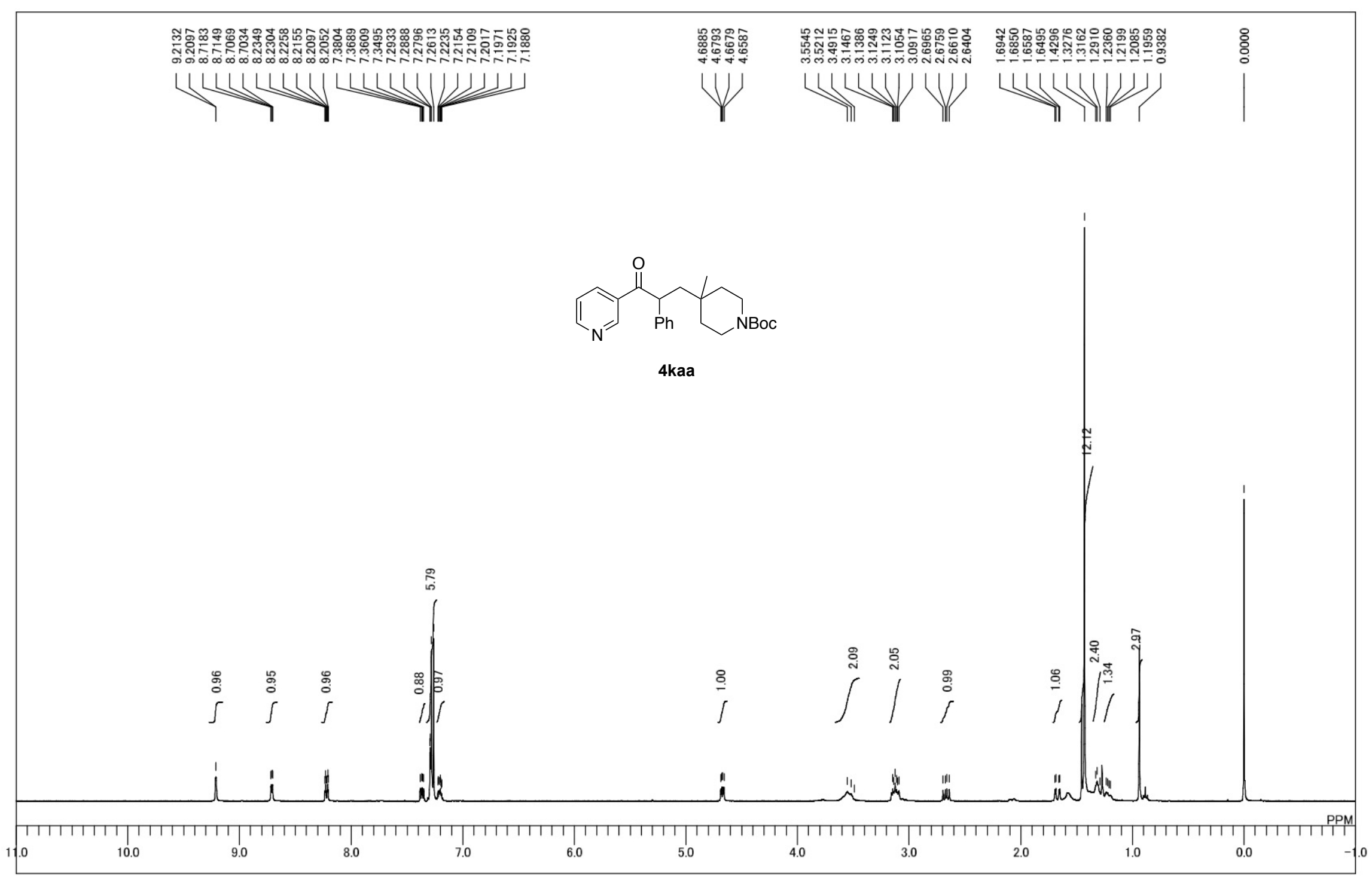

${ }^{1} \mathrm{H}$ NMR spectrum of $\mathbf{4 k a a}$ 


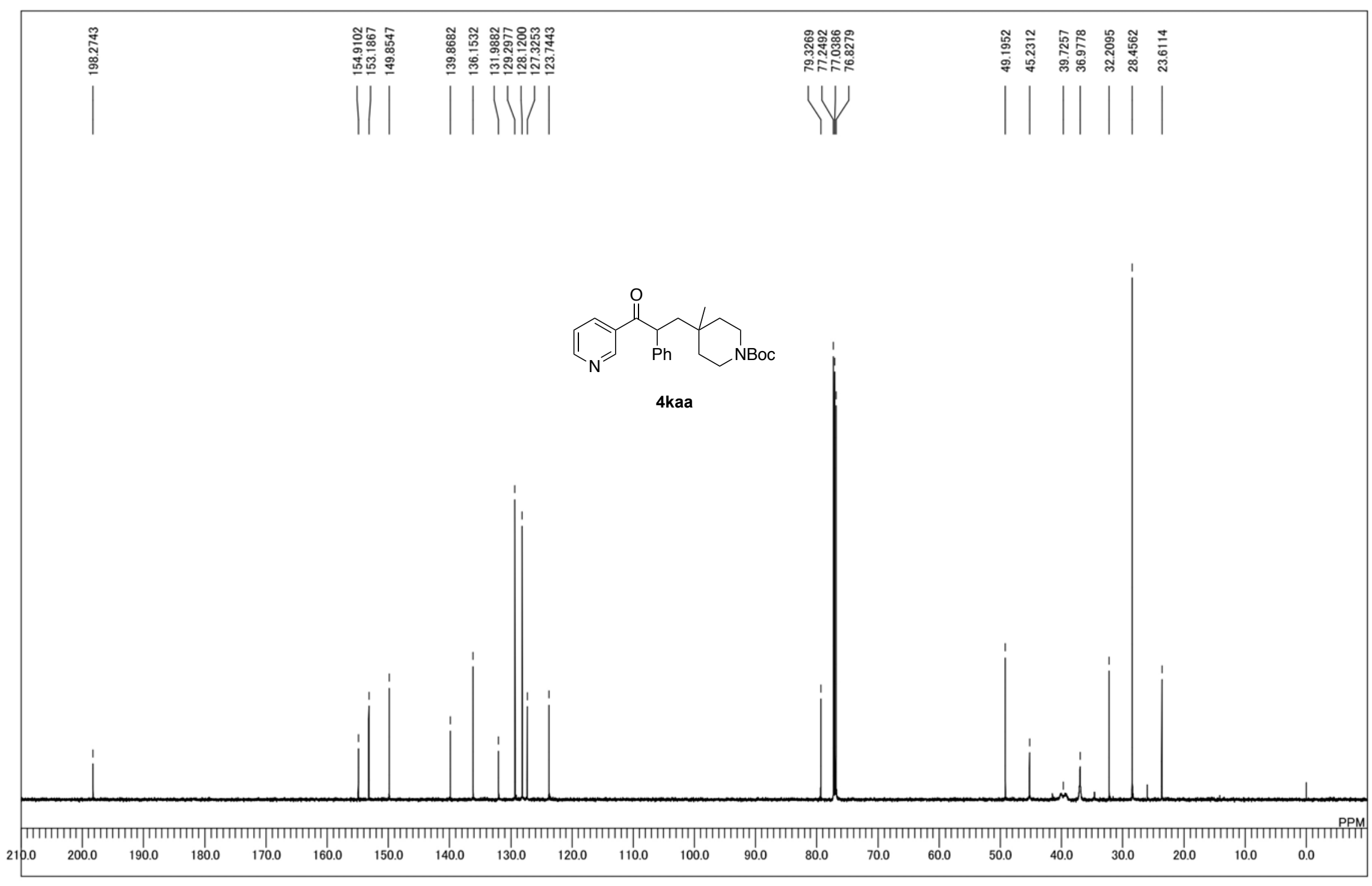

${ }^{13} \mathrm{C}$ NMR spectrum of $4 \mathbf{k a a}$ 


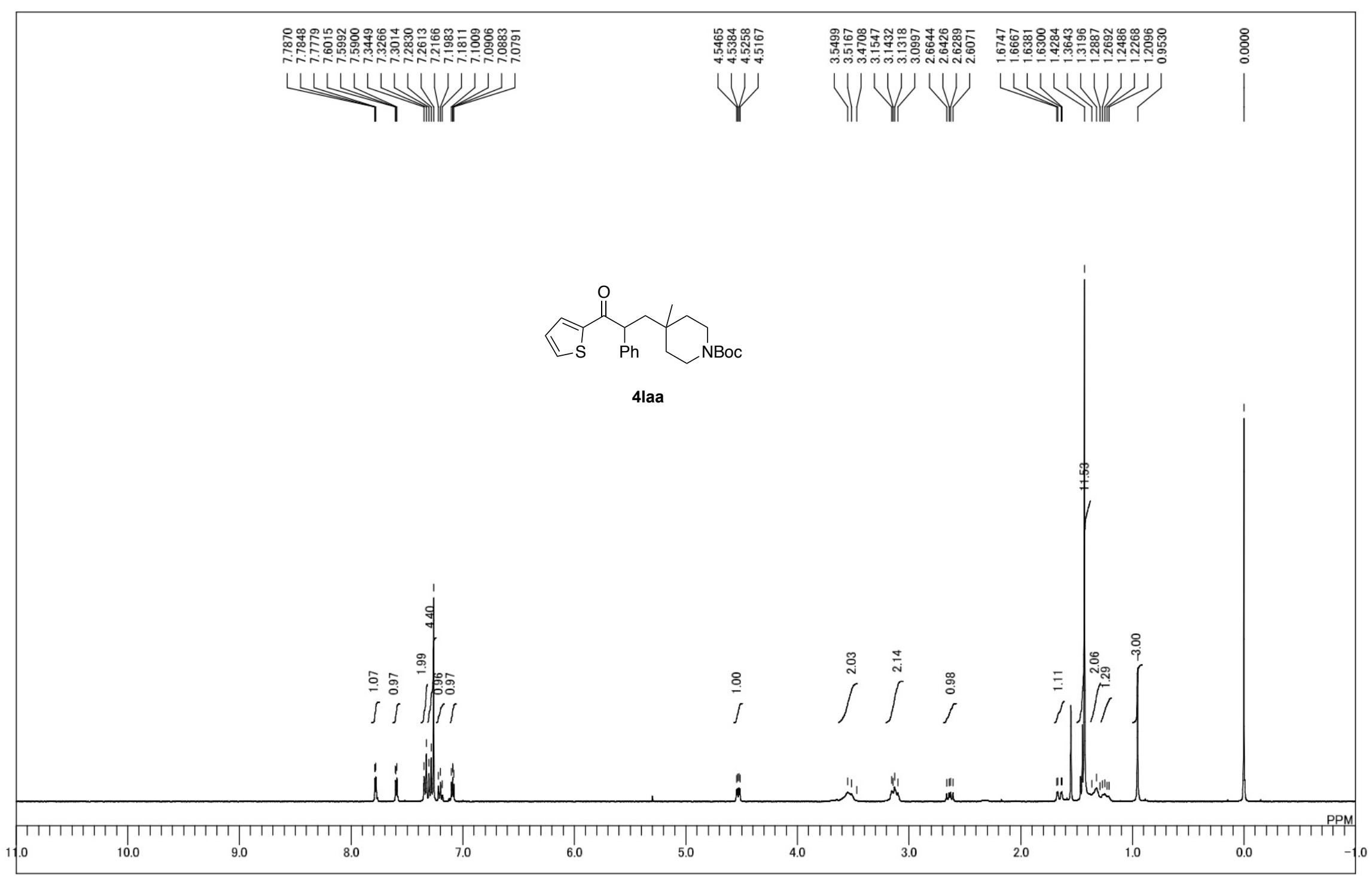

${ }^{1} \mathrm{H}$ NMR spectrum of 4laa 


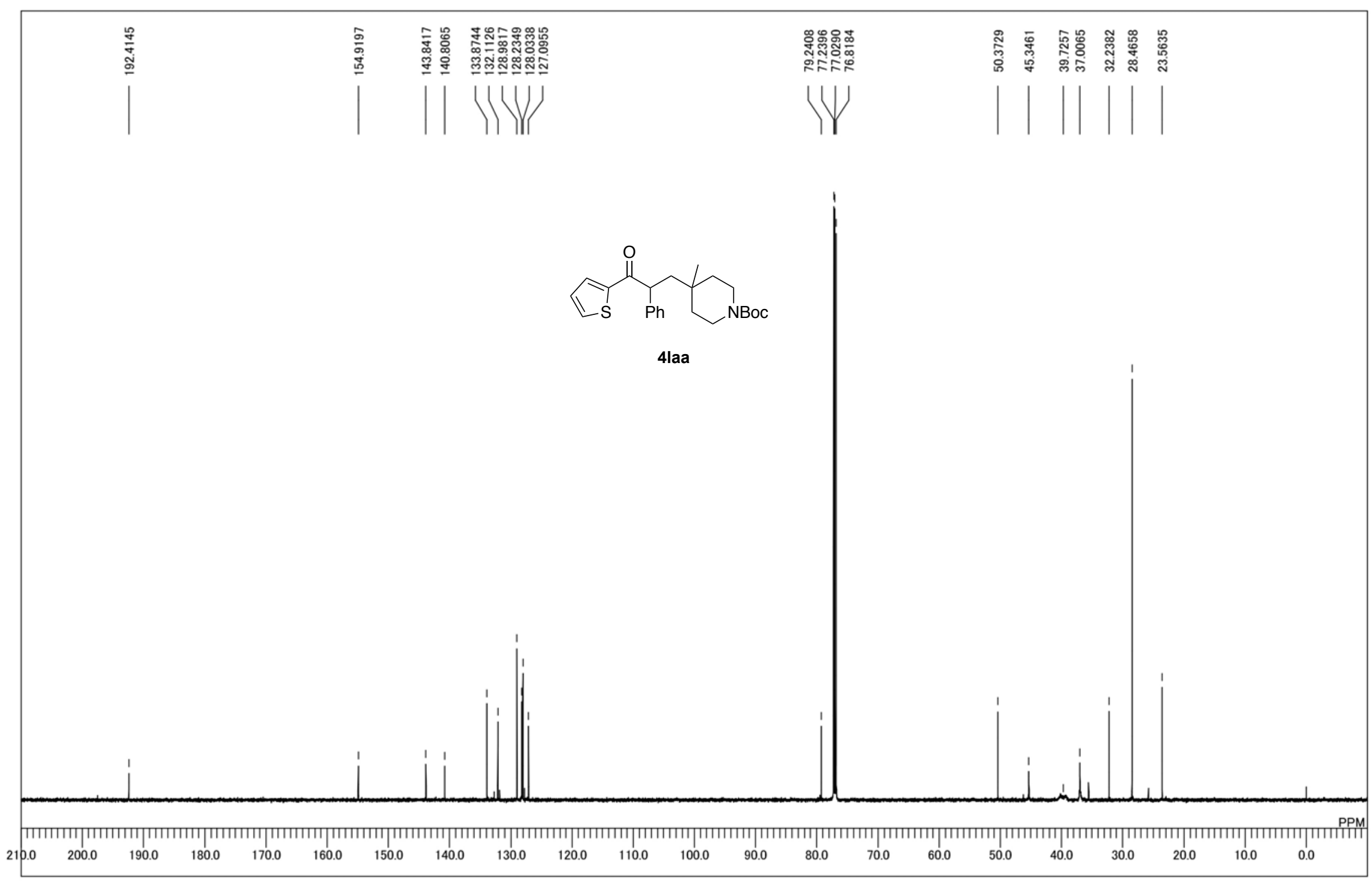

${ }^{13} \mathrm{C}$ NMR spectrum of 4laa 


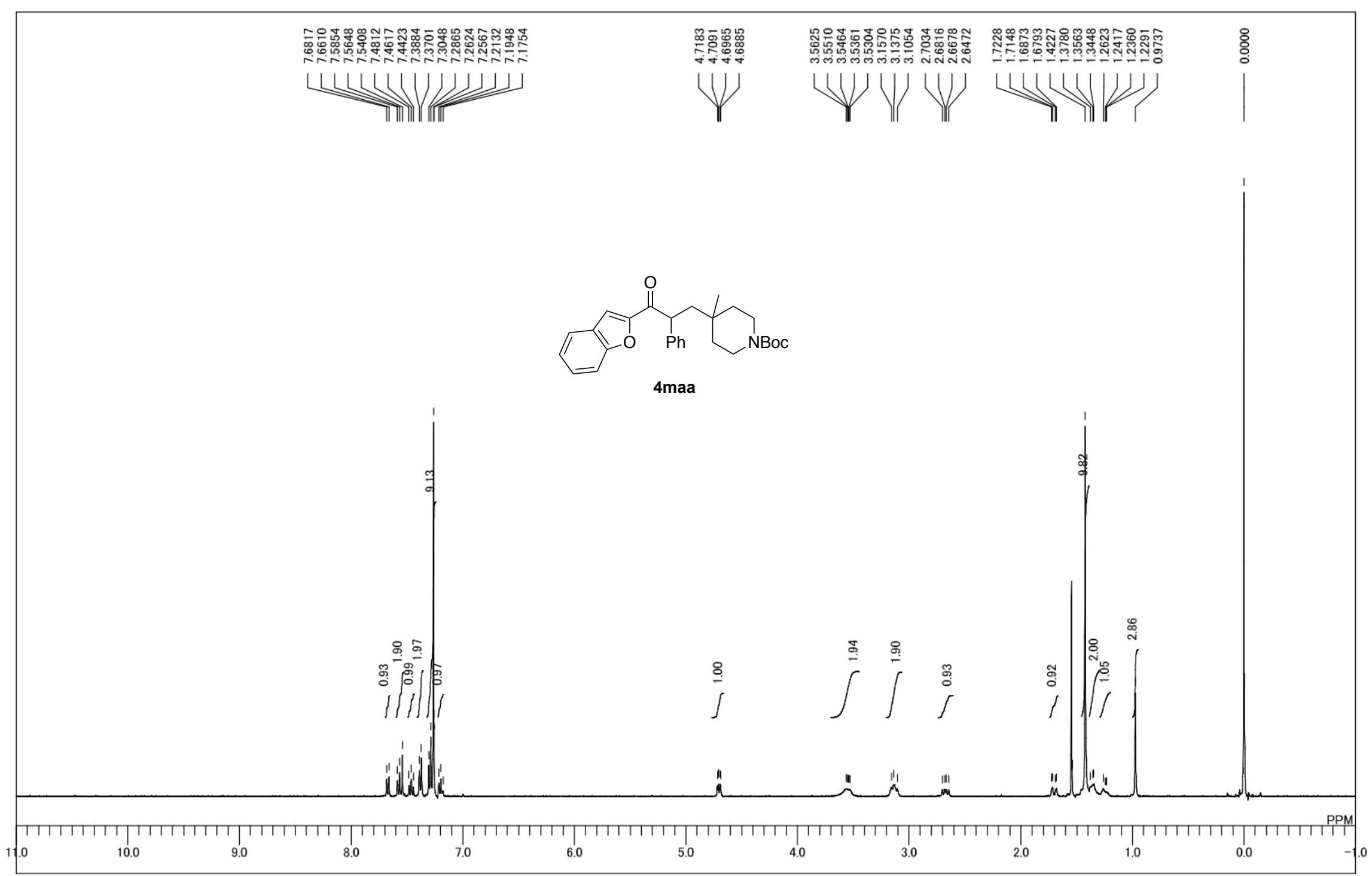

${ }^{1} \mathrm{H}$ NMR spectrum of $\mathbf{4 m a a}$ 


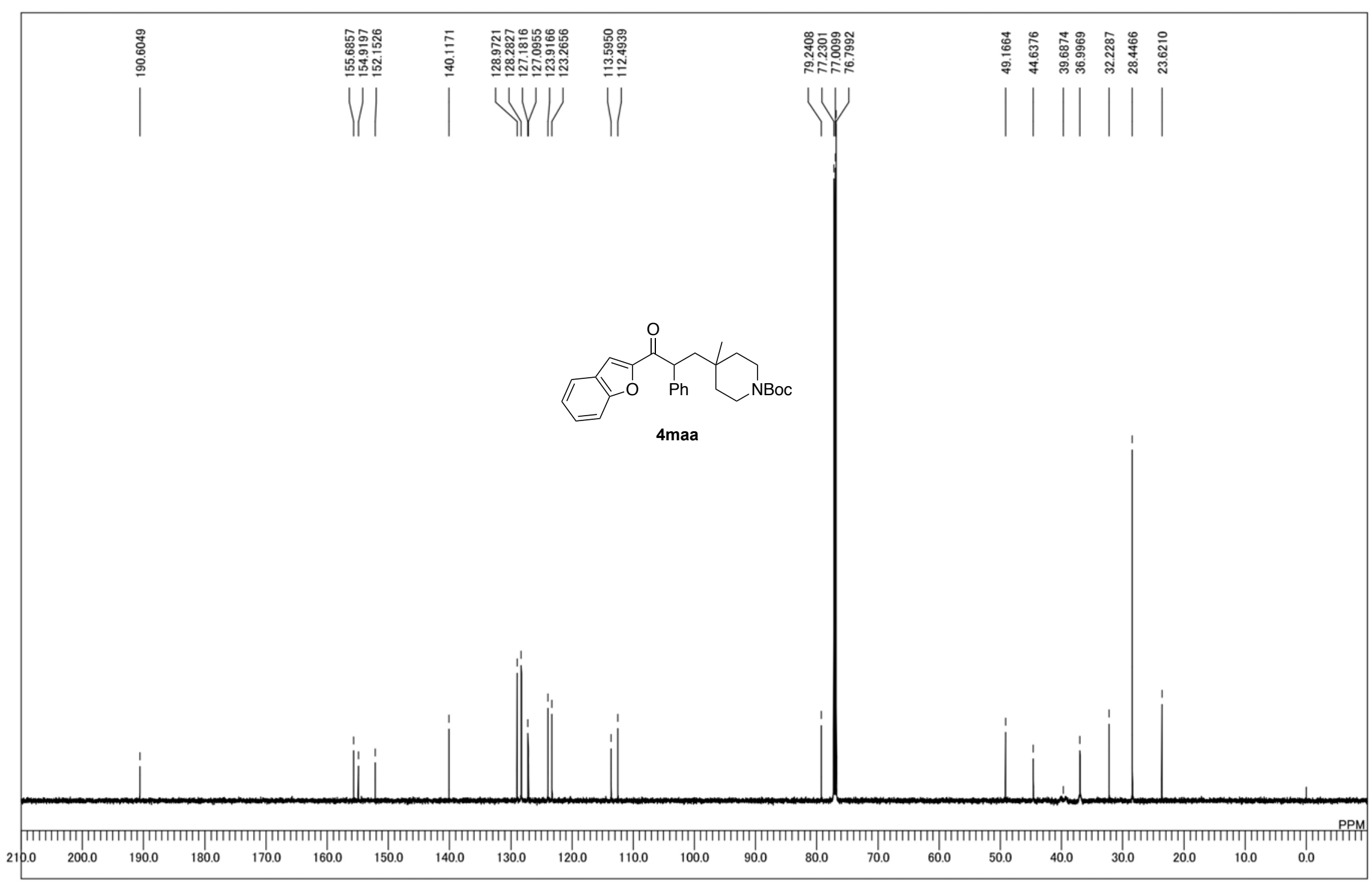

${ }^{13} \mathrm{C}$ NMR spectrum of 4 maa 


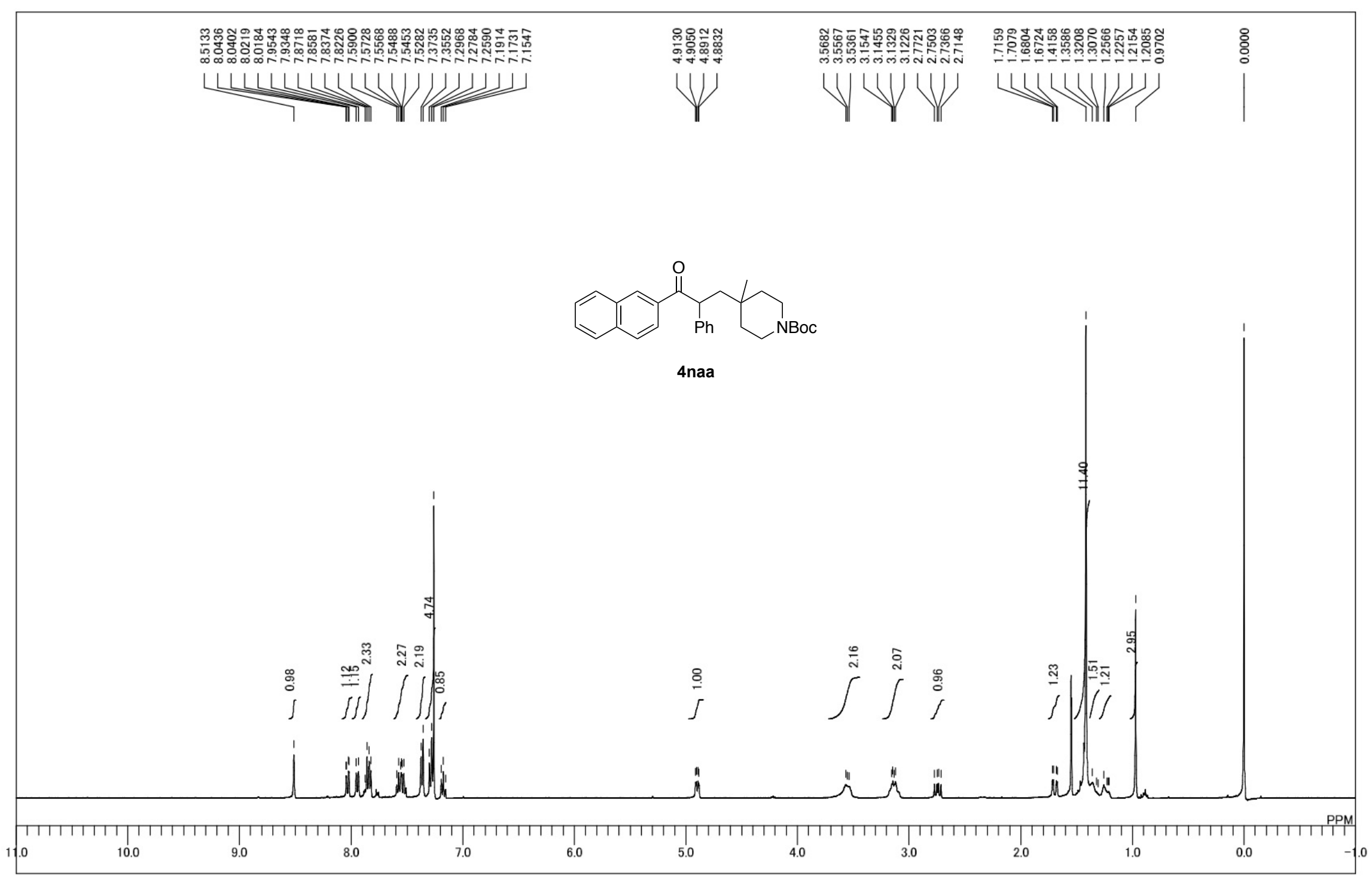

${ }^{1} \mathrm{H}$ NMR spectrum of 4 naa 


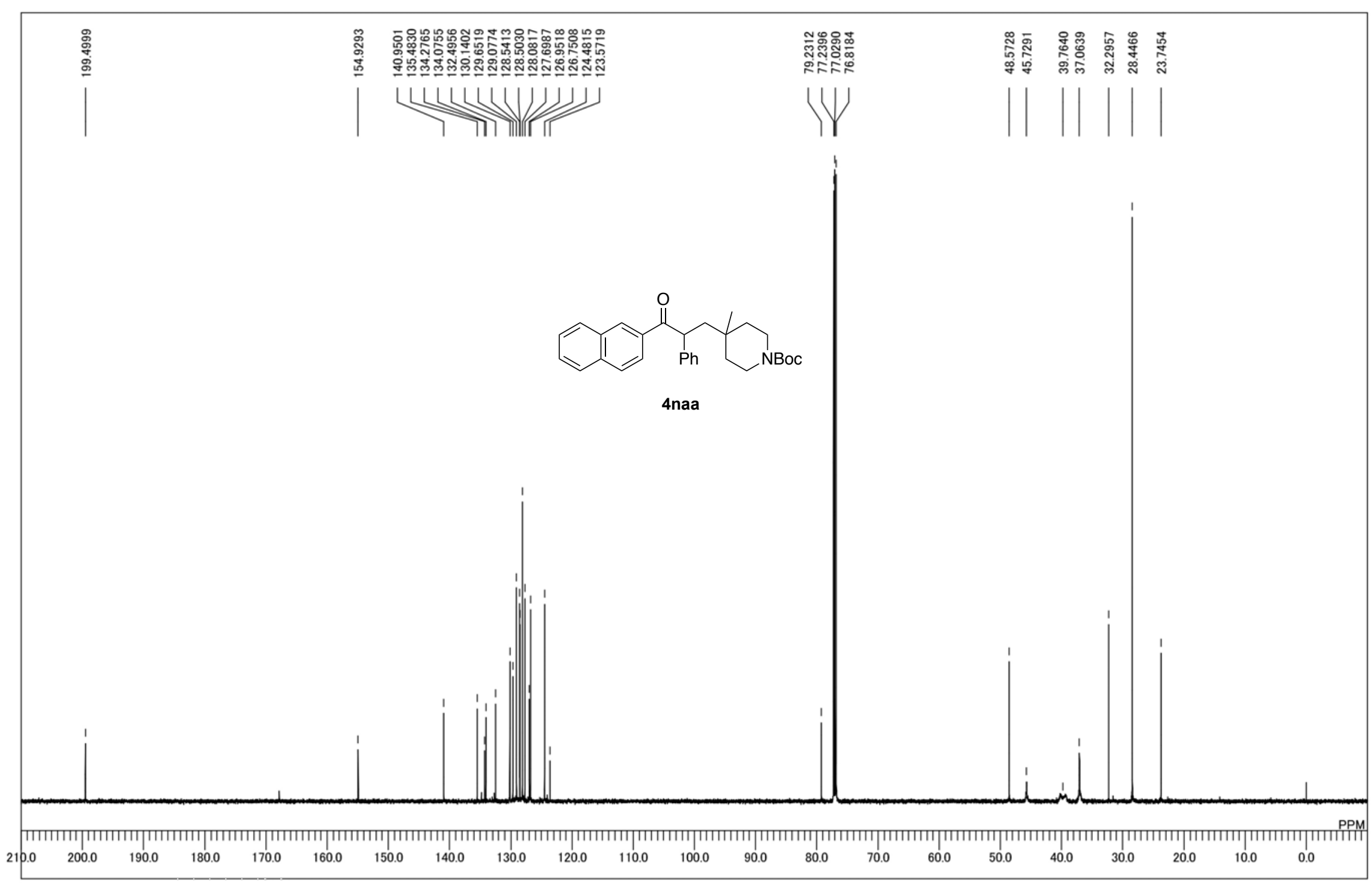

${ }^{13} \mathrm{C}$ NMR spectrum of 4 naa 


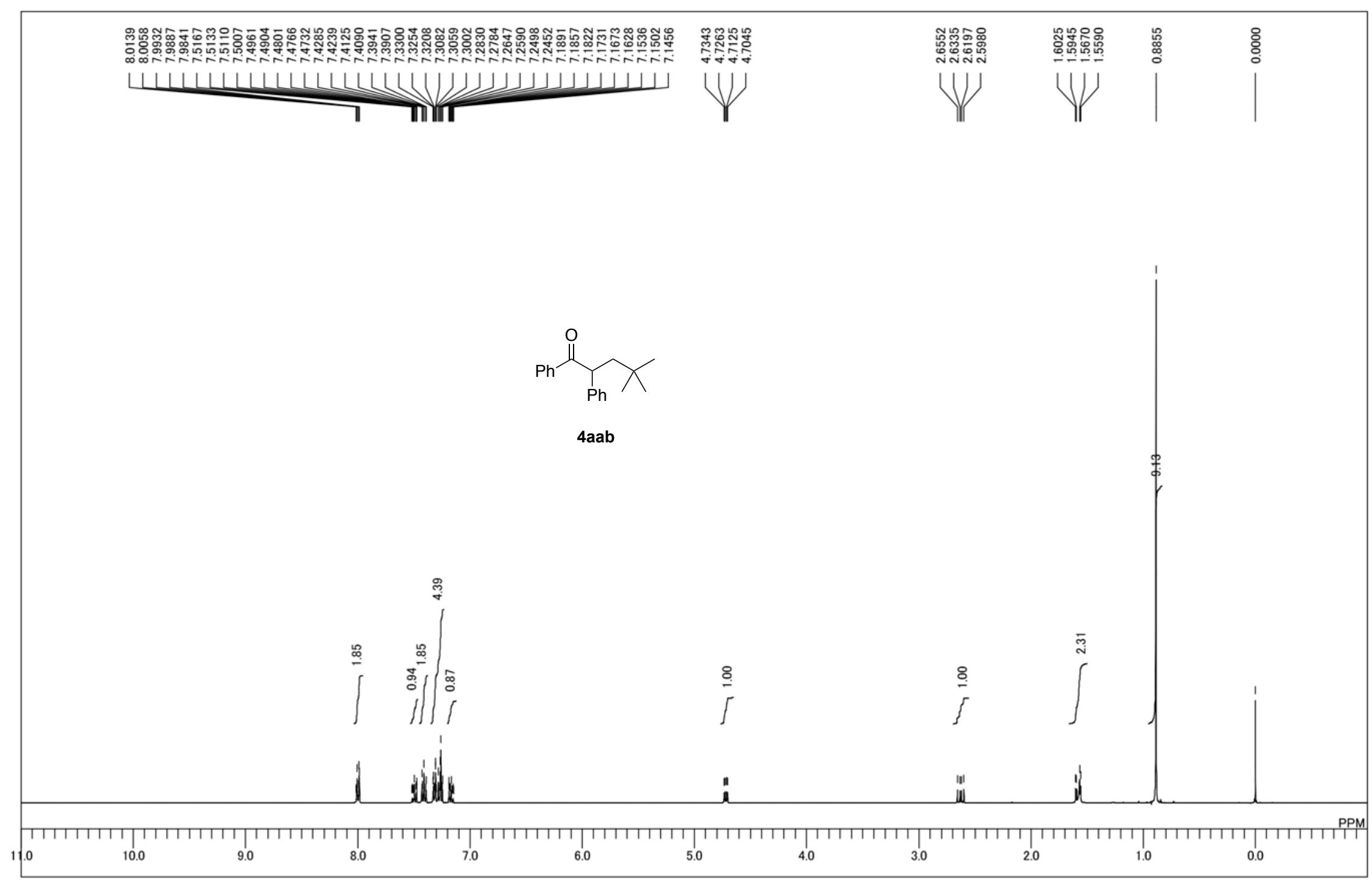

${ }^{1} \mathrm{H}$ NMR spectrum of $\mathbf{4 a a b}$ 


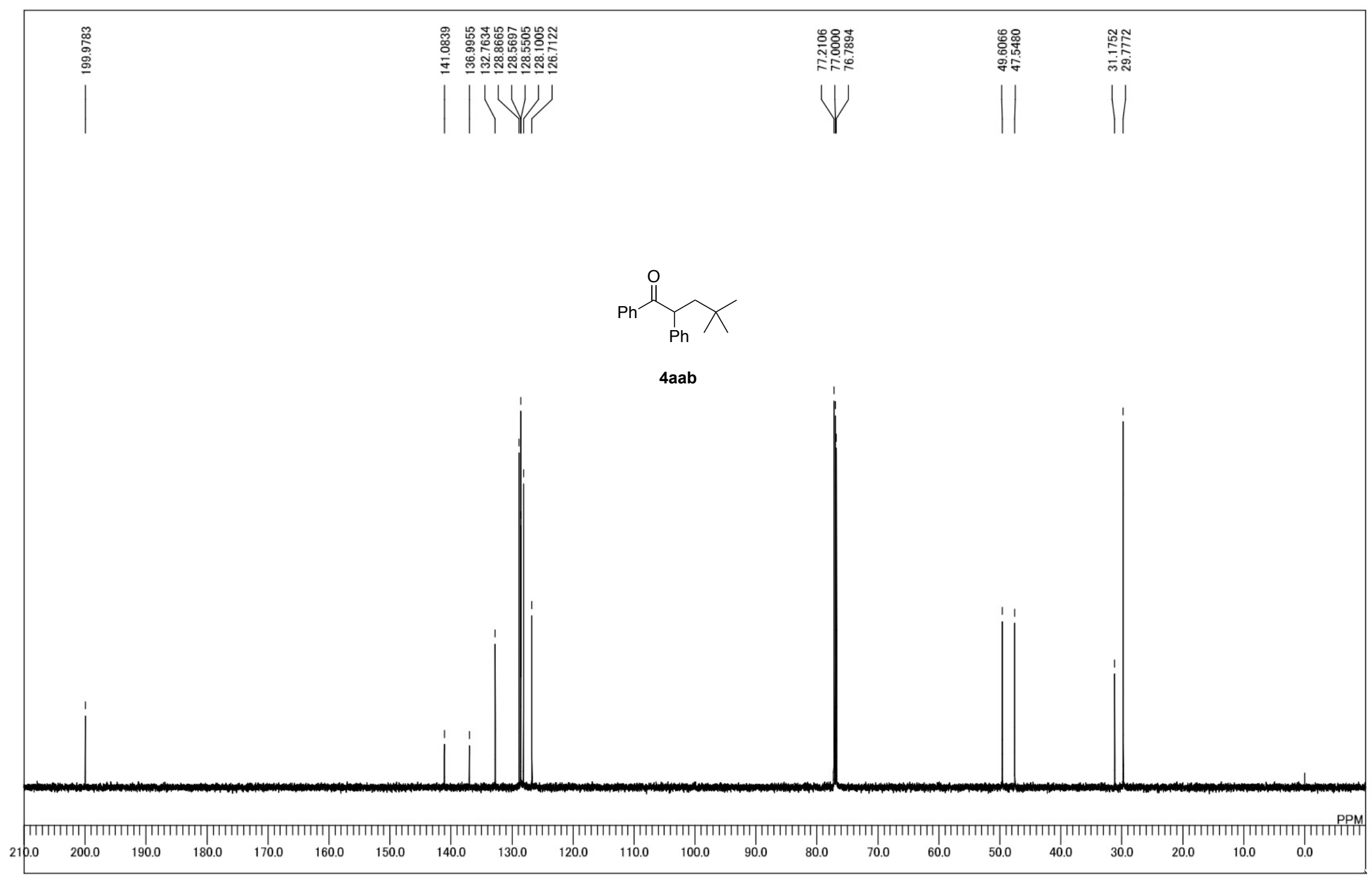

${ }^{13} \mathrm{C}$ NMR spectrum of $4 \mathbf{a a b}$ 


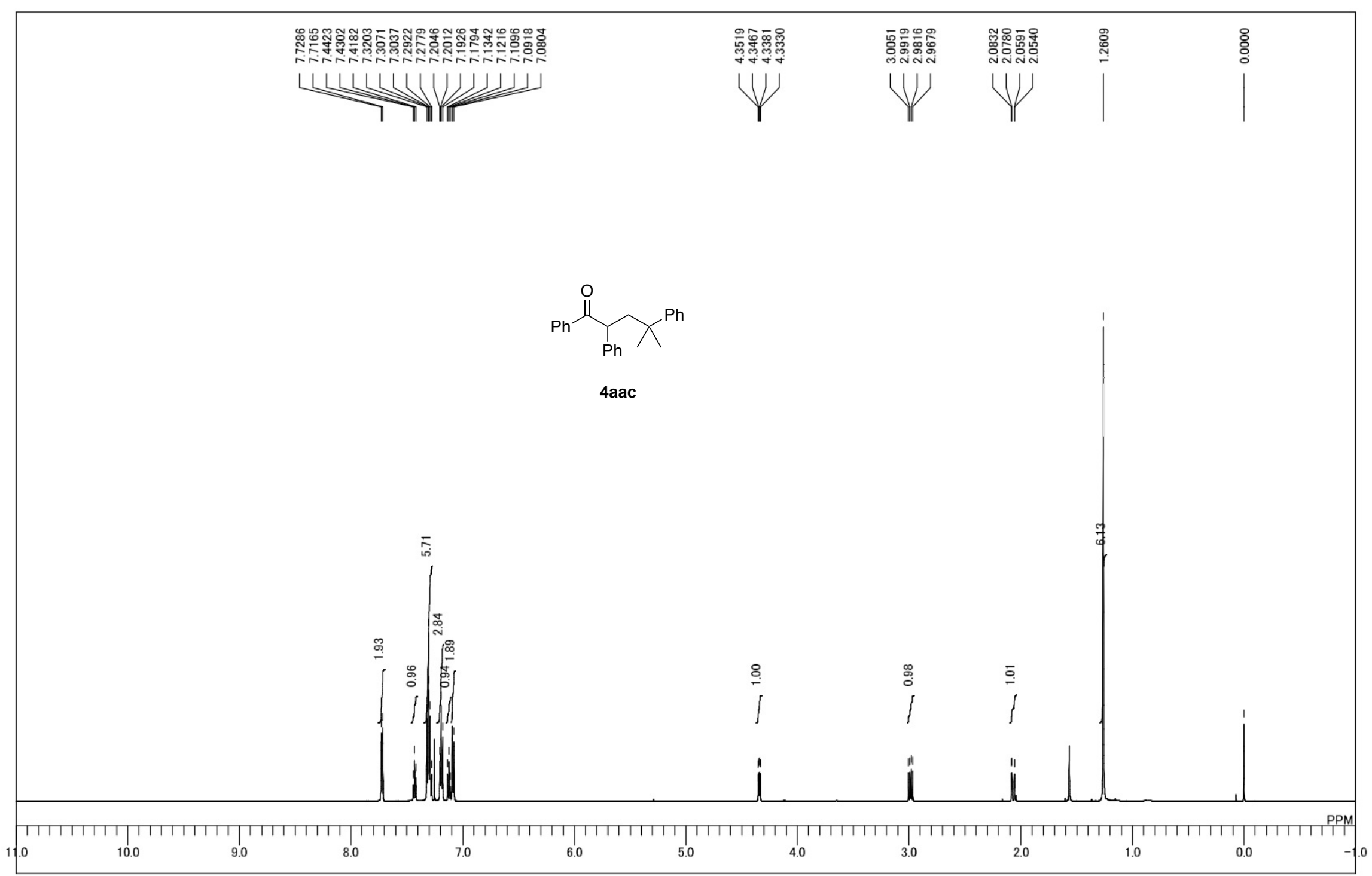

${ }^{1} \mathrm{H}$ NMR spectrum of 4 aac 


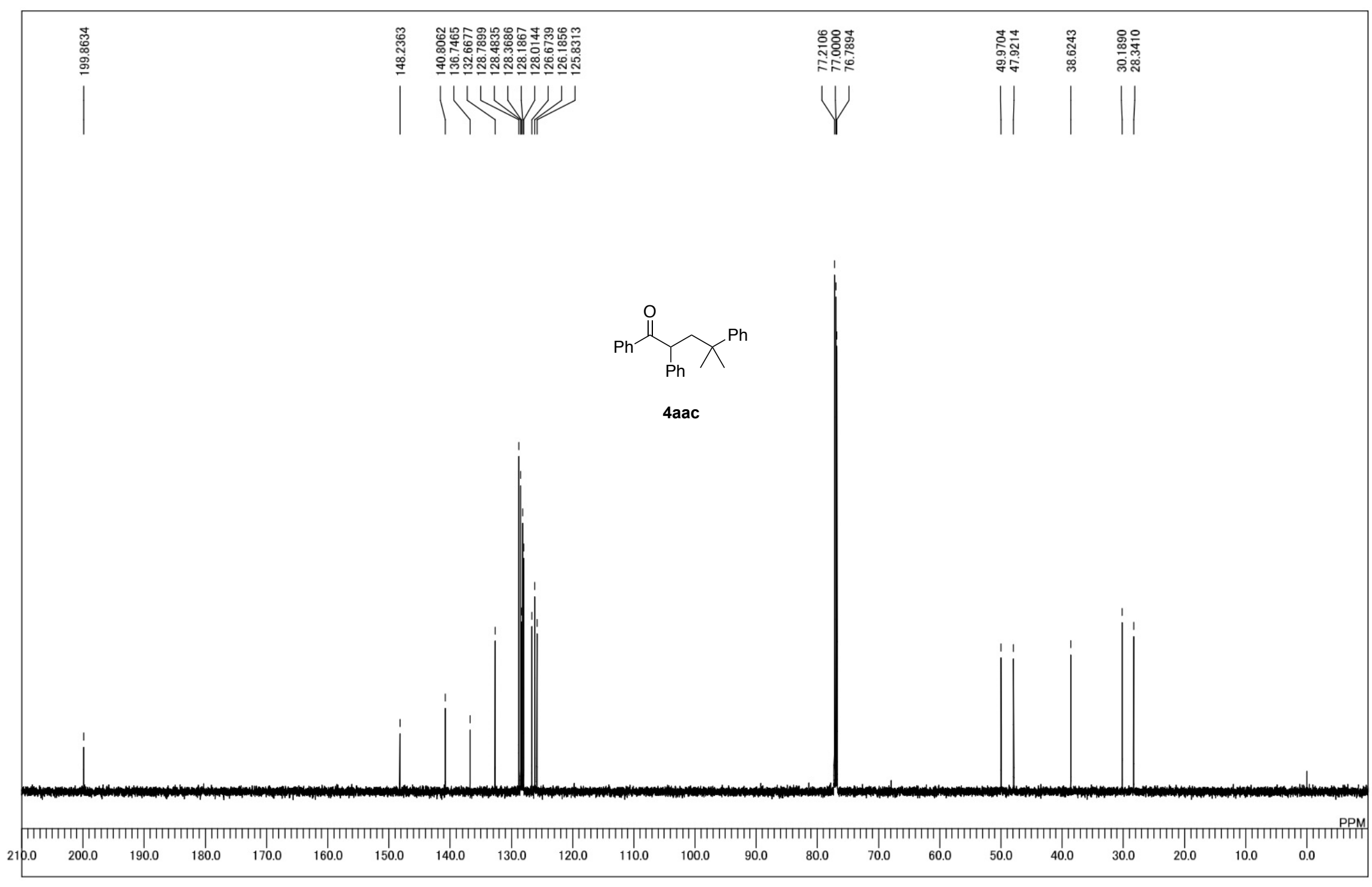

${ }^{13} \mathrm{C}$ NMR spectrum of $\mathbf{4 a a c}$ 


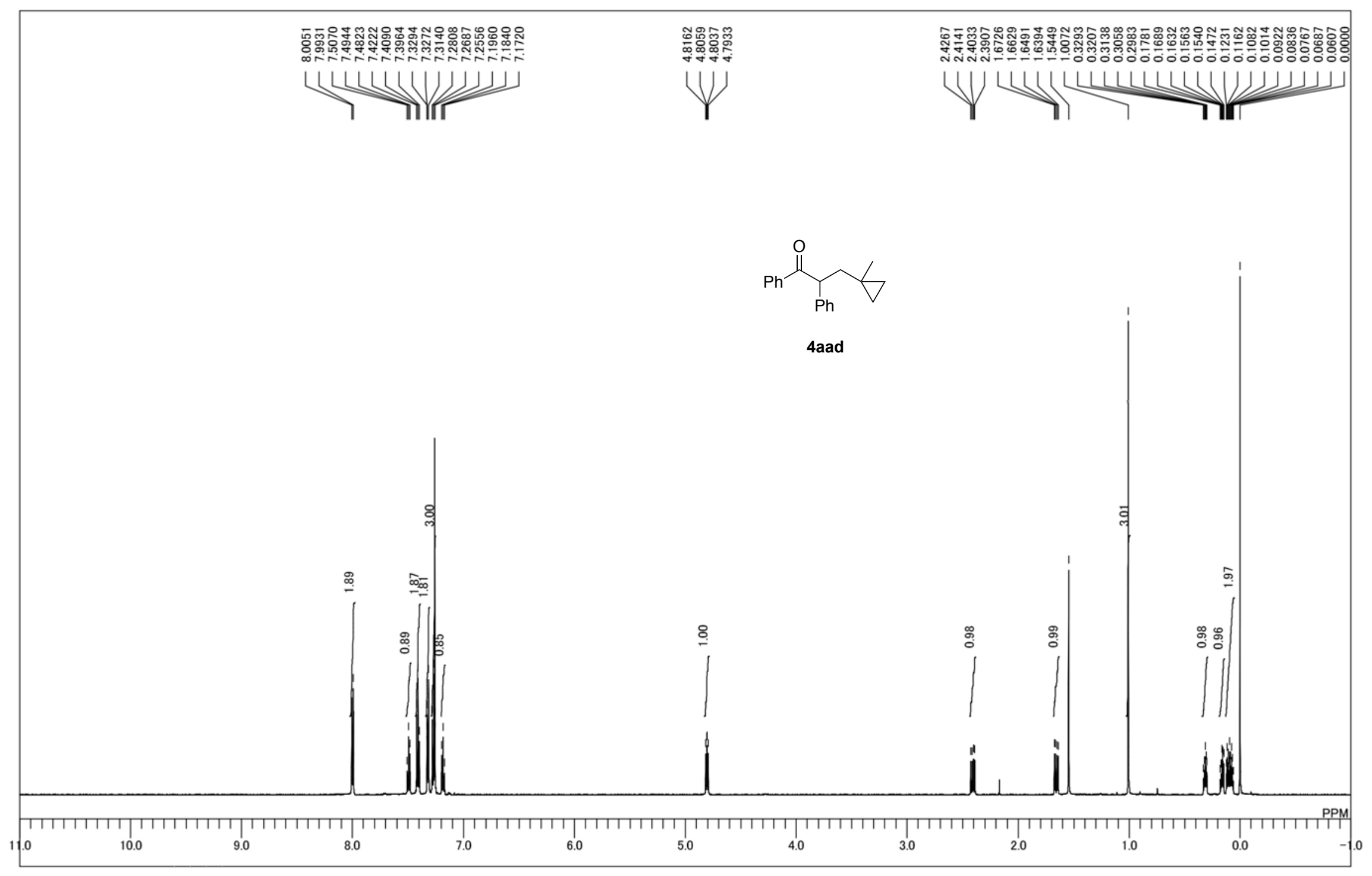

${ }^{1} \mathrm{H}$ NMR spectrum of $\mathbf{4 a a d}$ 


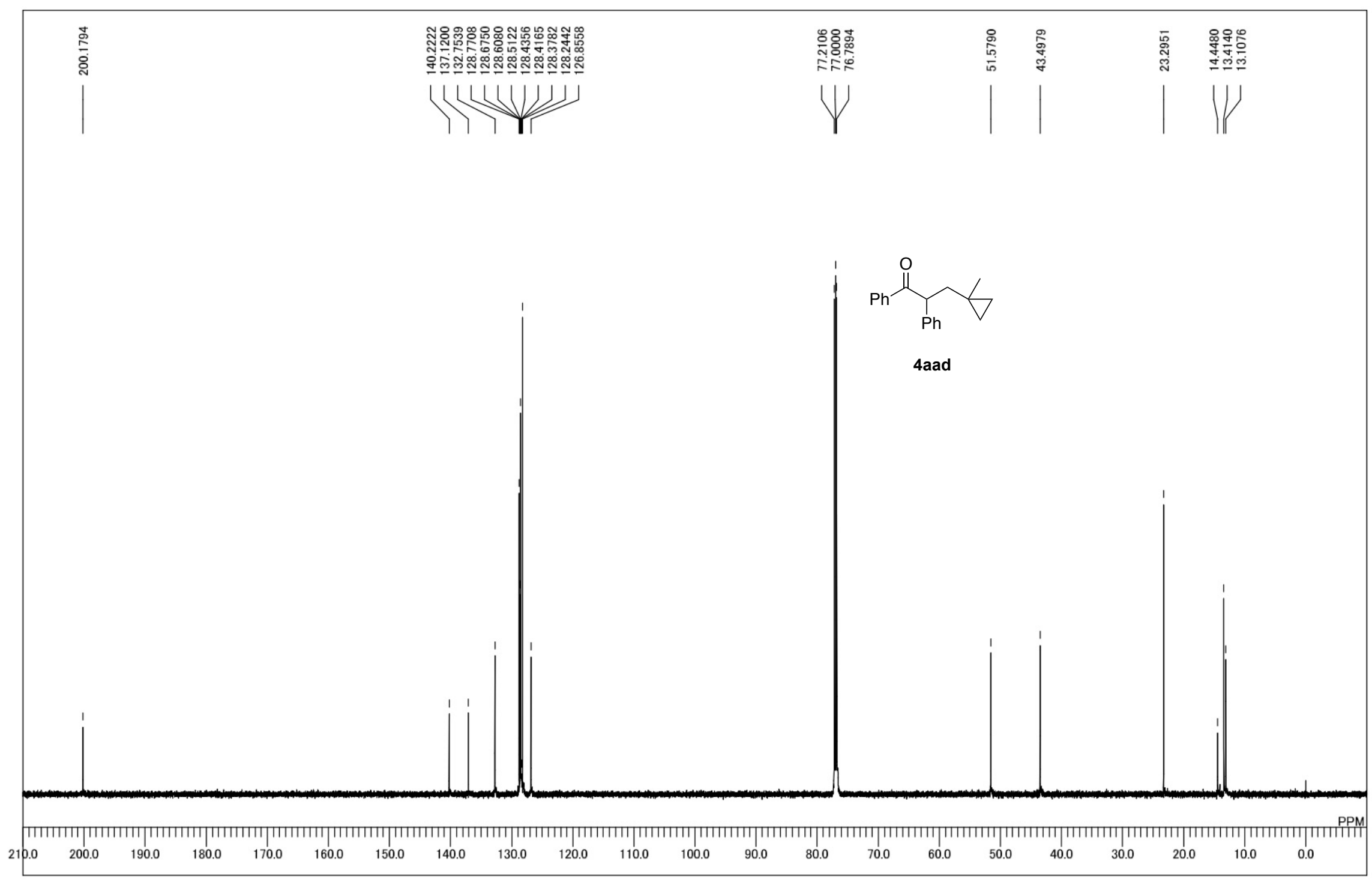

${ }^{13} \mathrm{C}$ NMR spectrum of $4 \mathbf{a a d}$ 


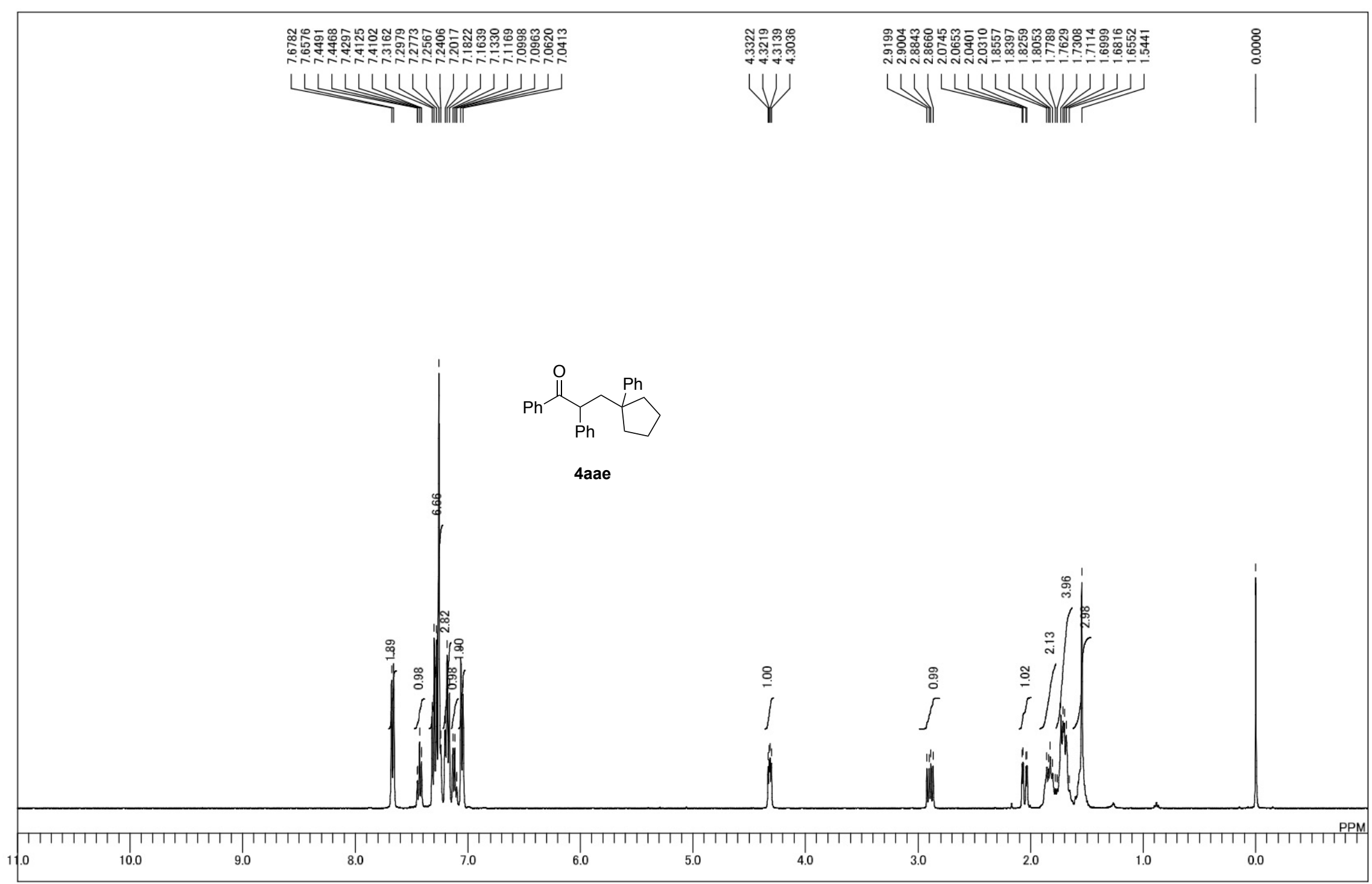

${ }^{1} \mathrm{H}$ NMR spectrum of 4aae 


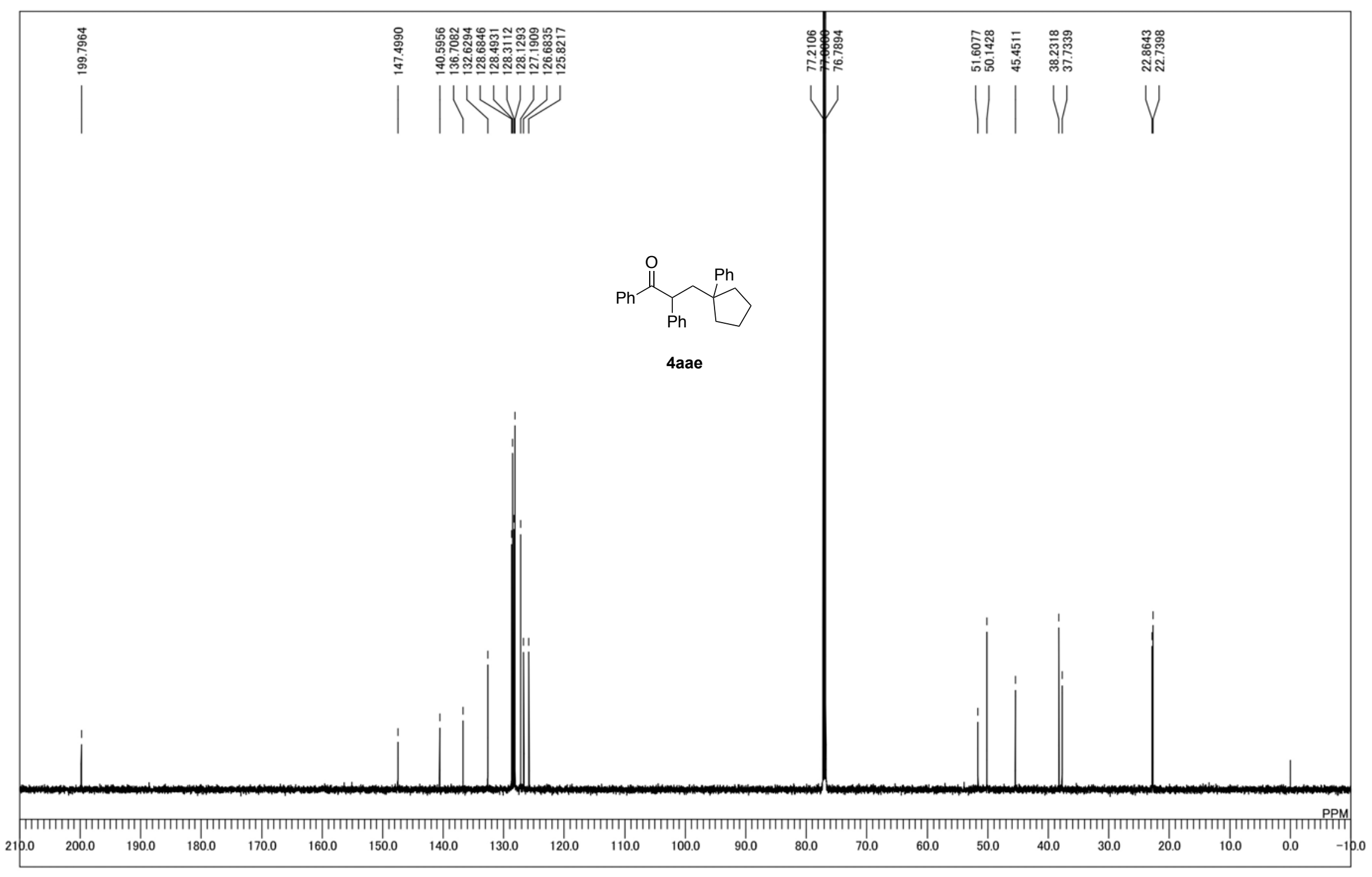

${ }^{13} \mathrm{C}$ NMR spectrum of $\mathbf{4 a a e}$ 


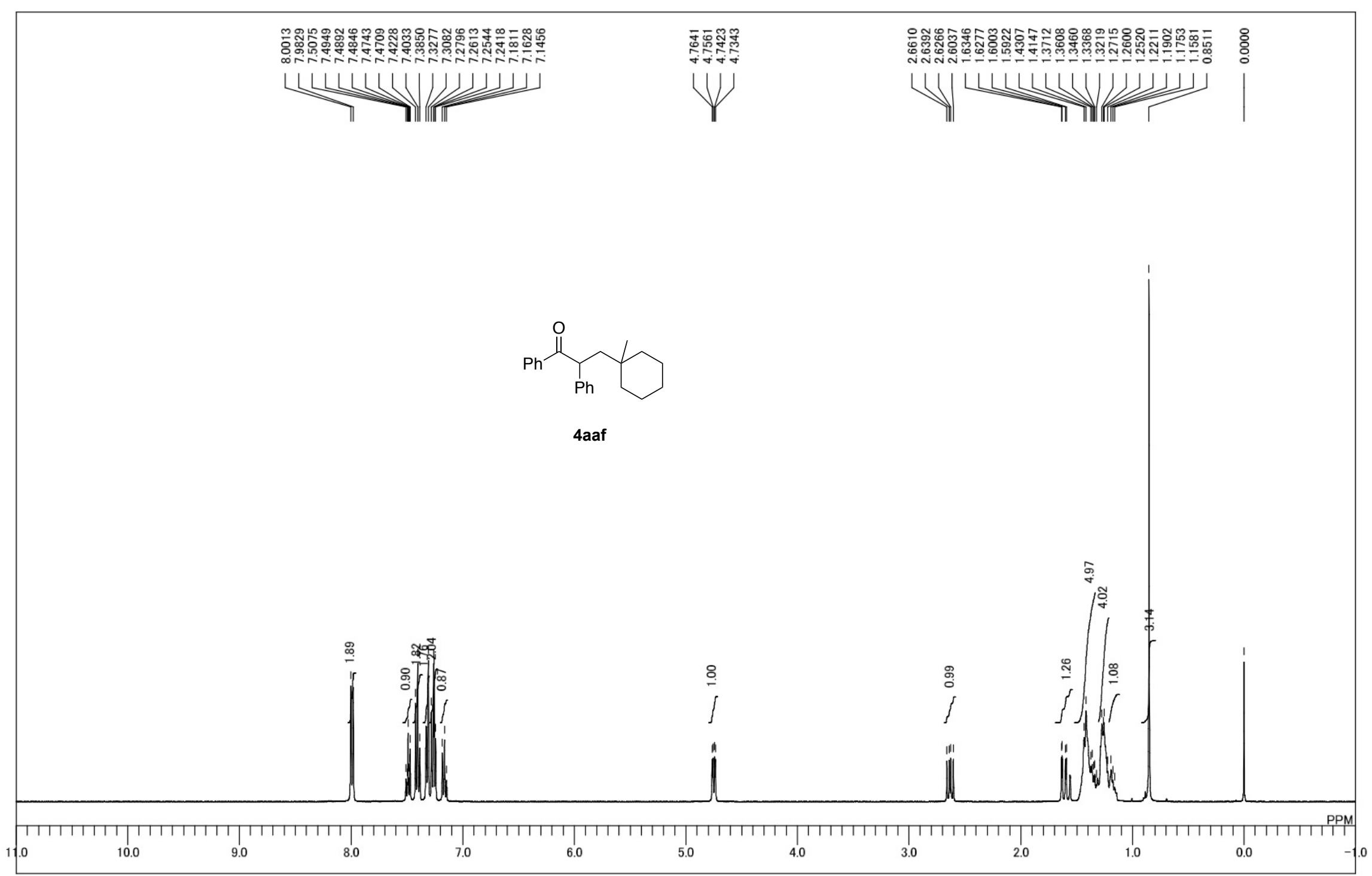

${ }^{1} \mathrm{H}$ NMR spectrum of $\mathbf{4 a a f}$ 


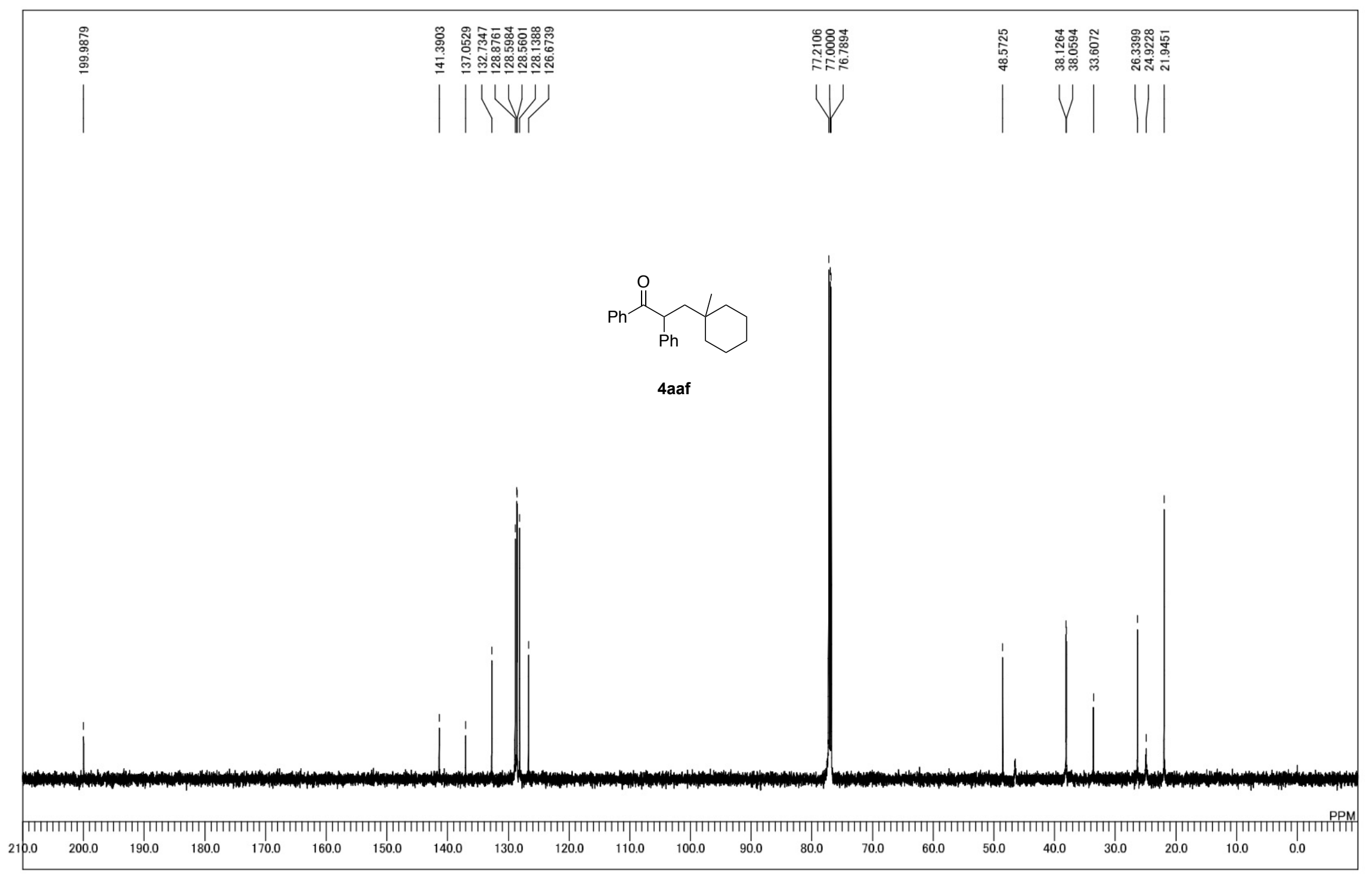

${ }^{13} \mathrm{C}$ NMR spectrum of $\mathbf{4 a a f}$ 


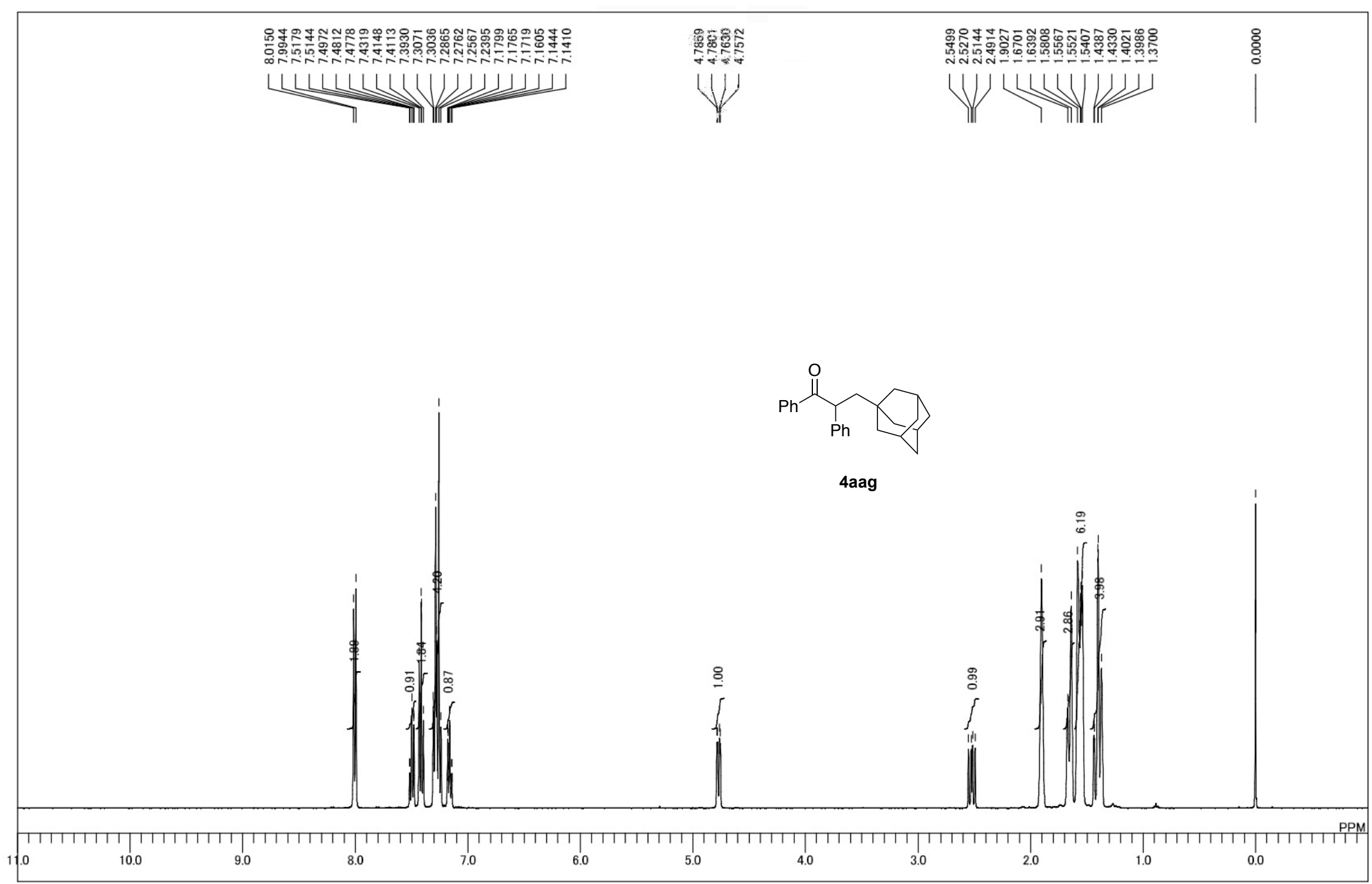

${ }^{1} \mathrm{H}$ NMR spectrum of $\mathbf{4 a a g}$ 


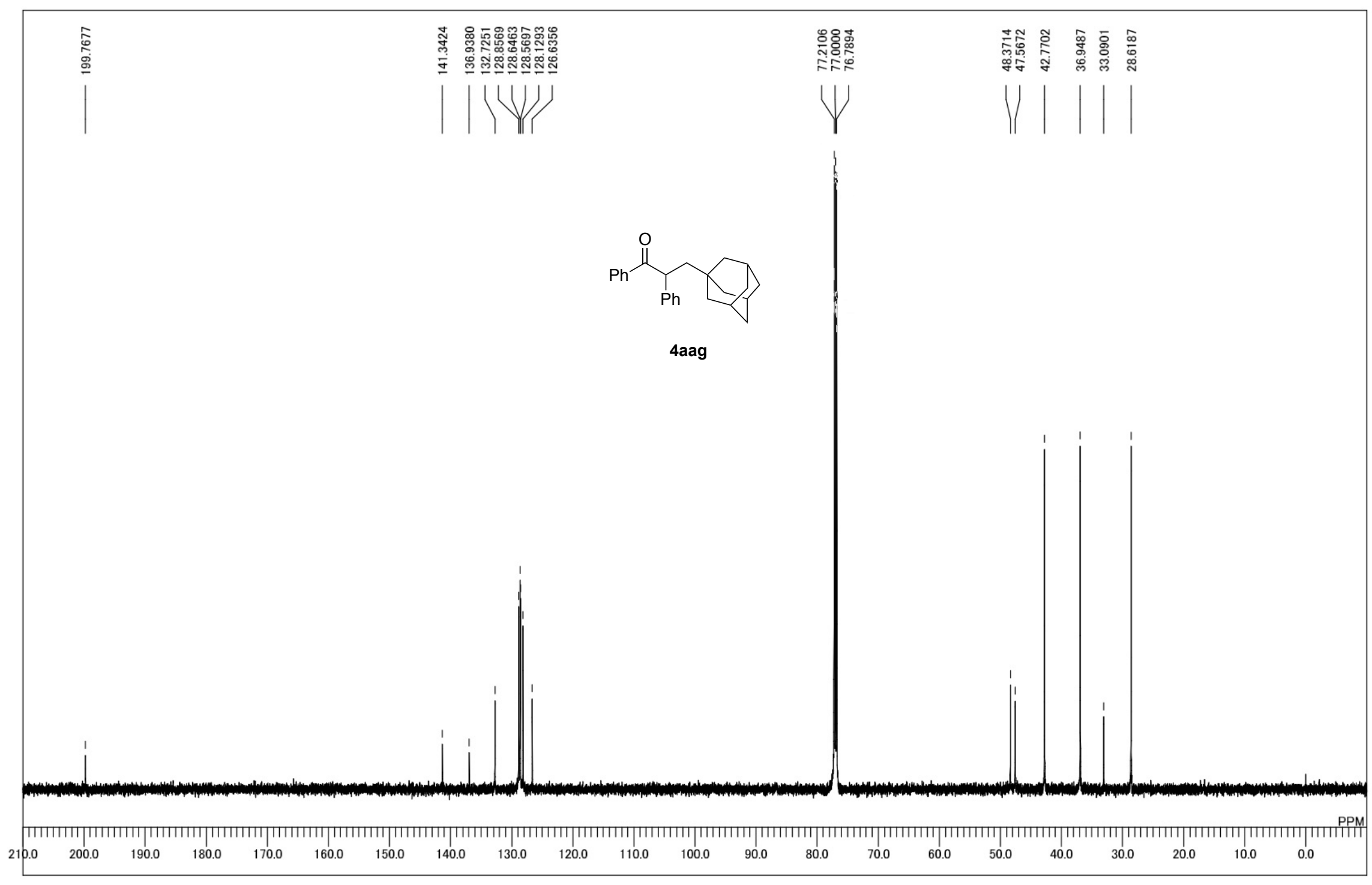

${ }^{13} \mathrm{C}$ NMR spectrum of $\mathbf{4 a a g}$ 


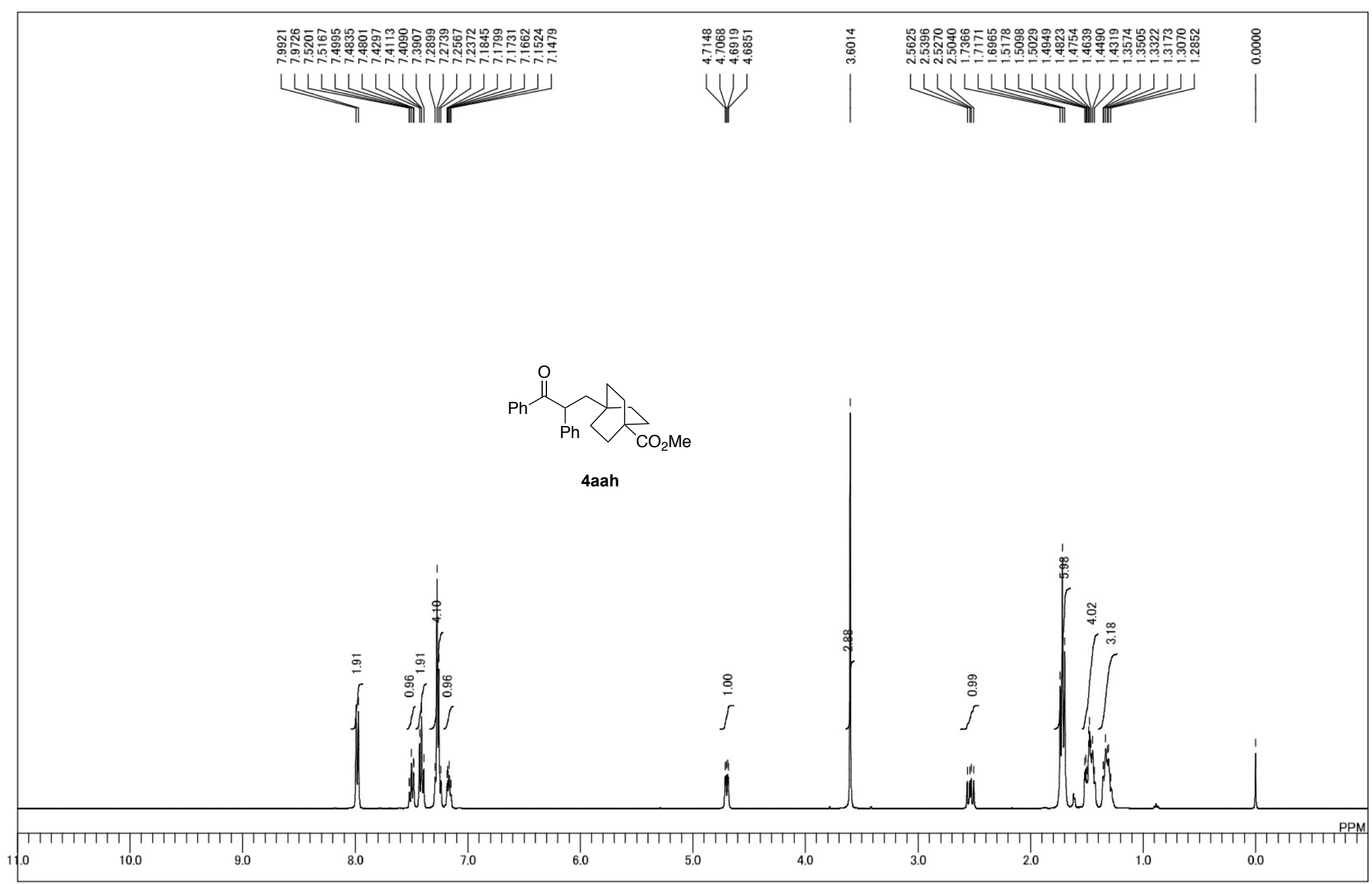

${ }^{1} \mathrm{H}$ NMR spectrum of $\mathbf{4 a a h}$ 


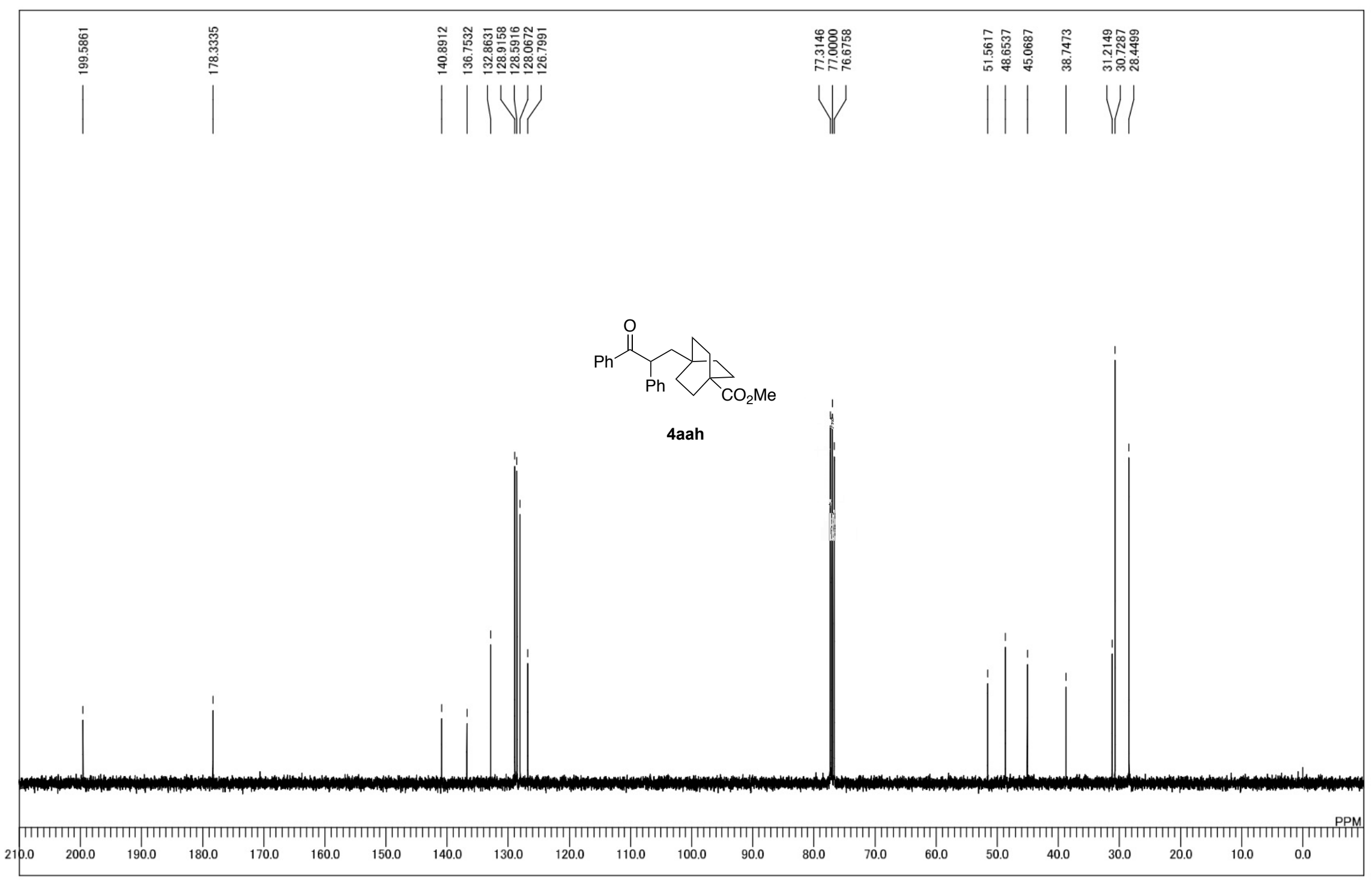

${ }^{13} \mathrm{C}$ NMR spectrum of $\mathbf{4 a a h}$

S63 


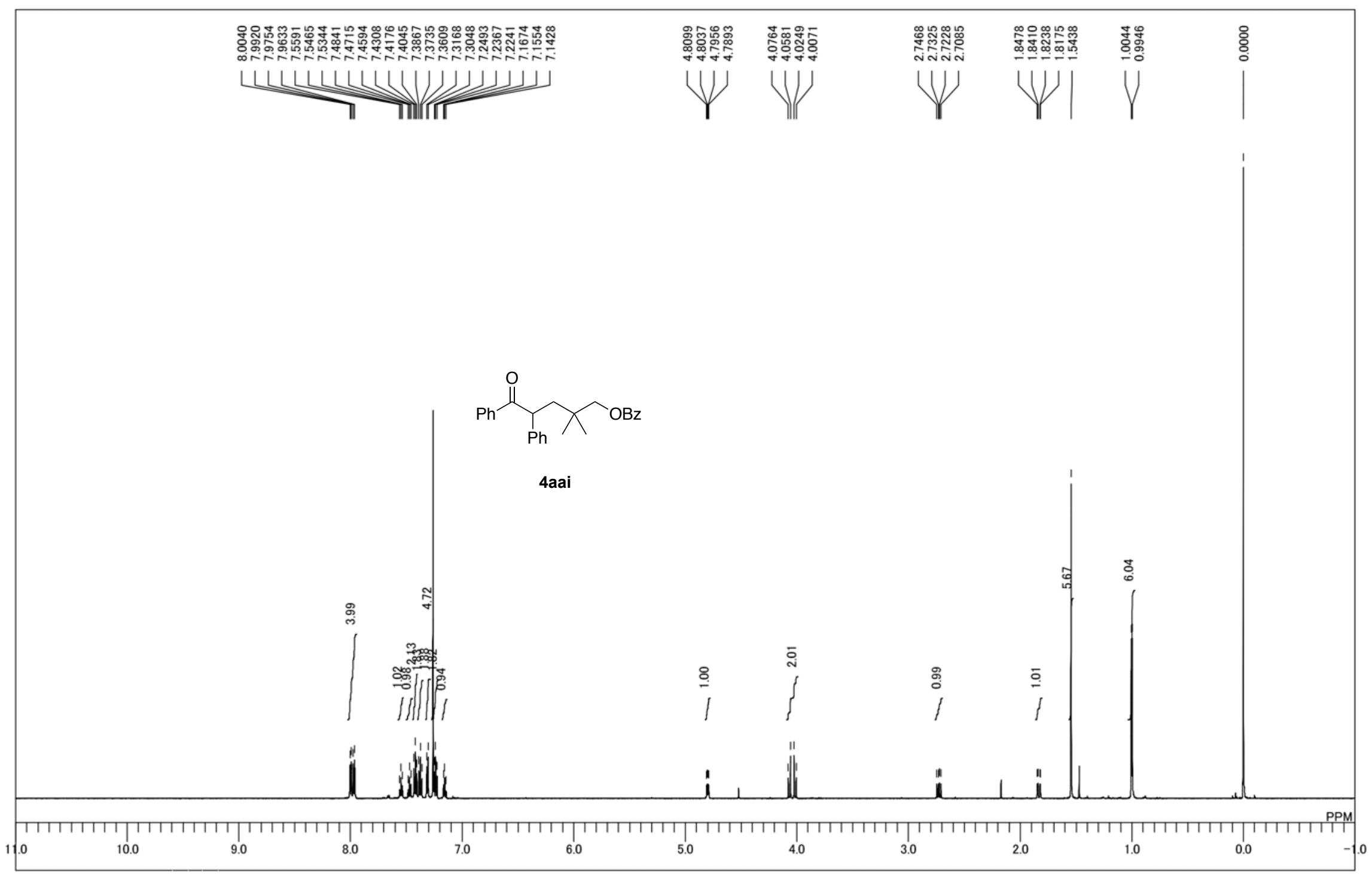

${ }^{1} \mathrm{H}$ NMR spectrum of 4aai 


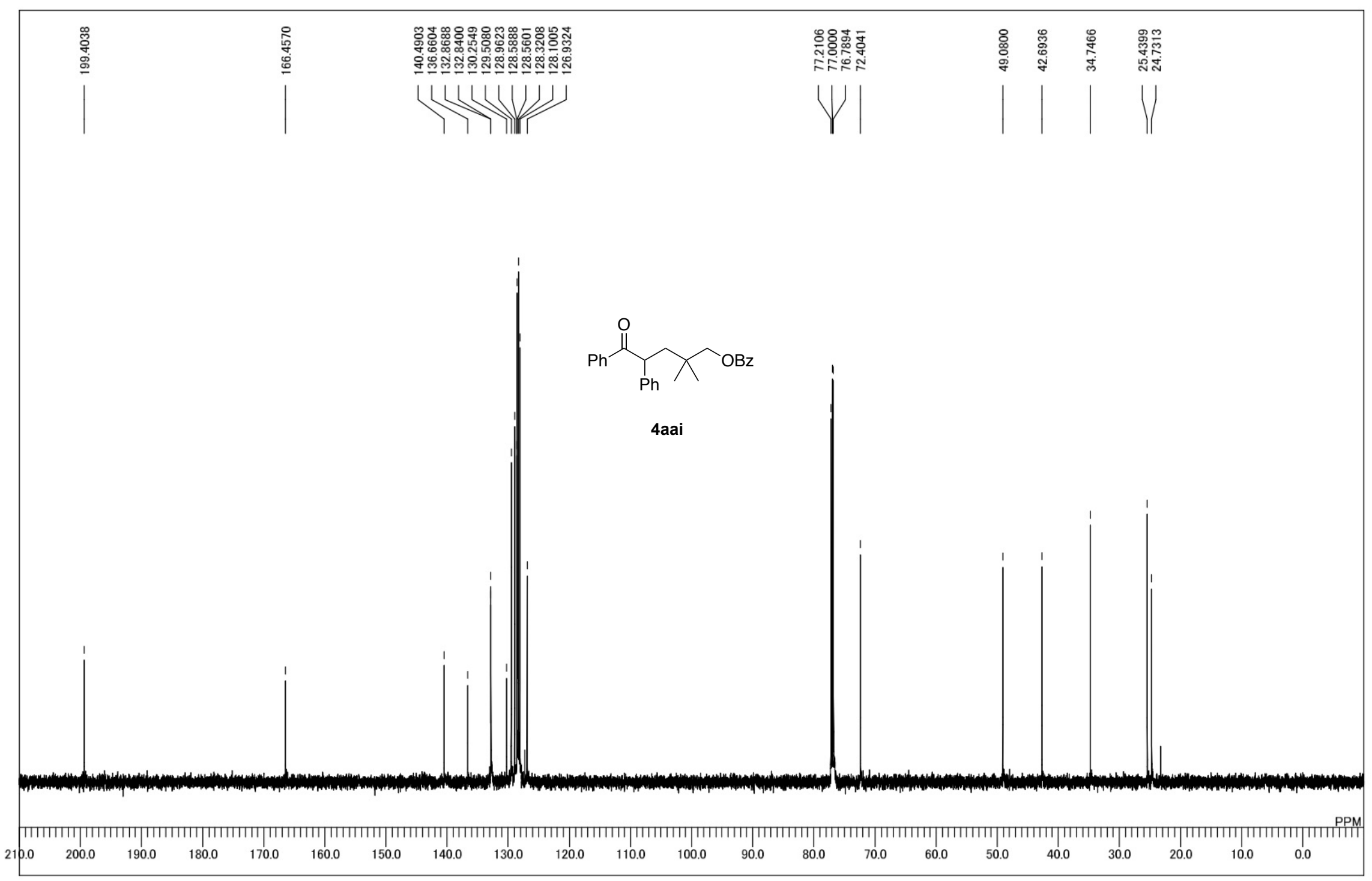

${ }^{13} \mathrm{C}$ NMR spectrum of $\mathbf{4 a a i}$

S65 


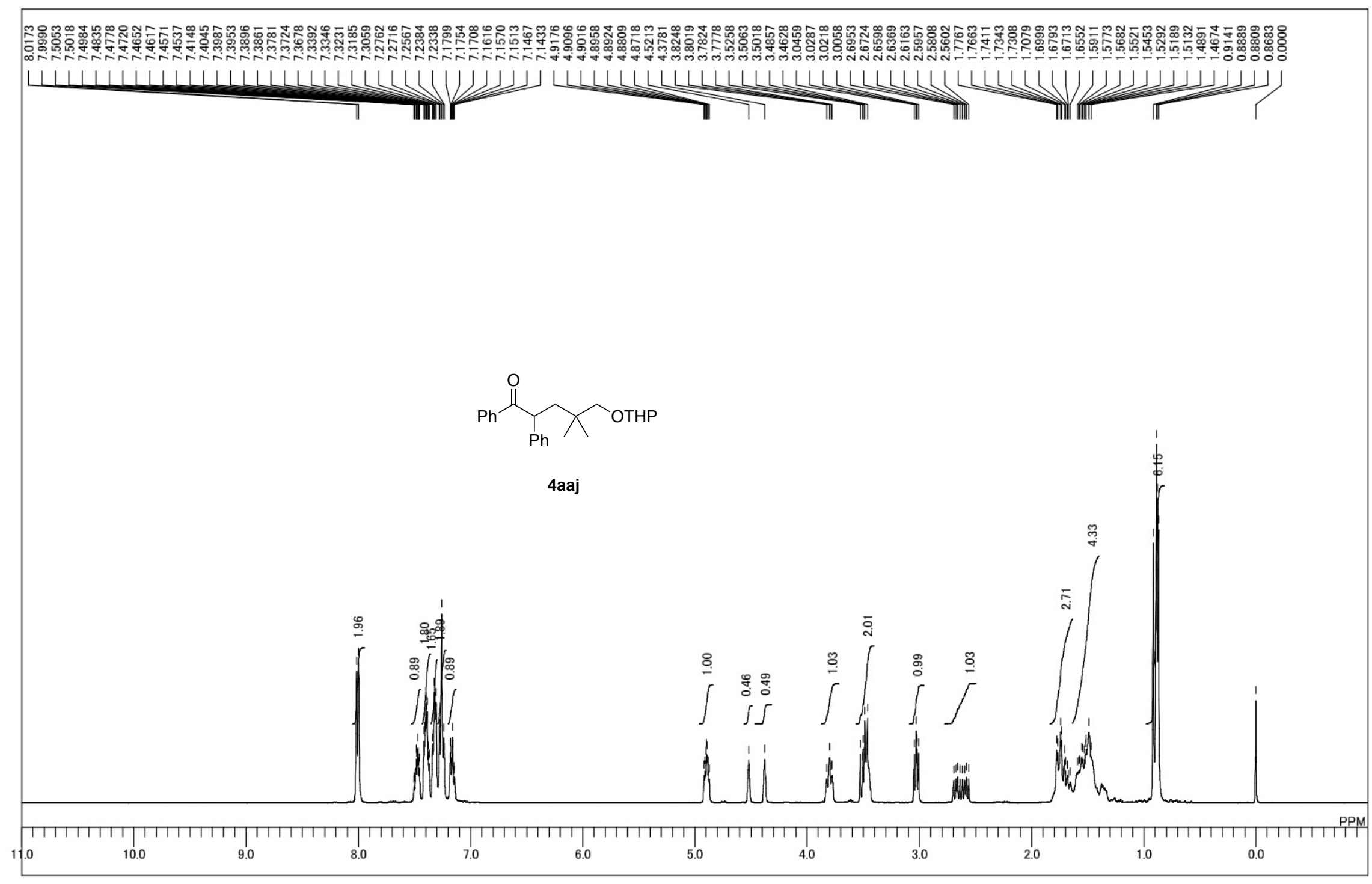

${ }^{1} \mathrm{H}$ NMR spectrum of 4aaj 


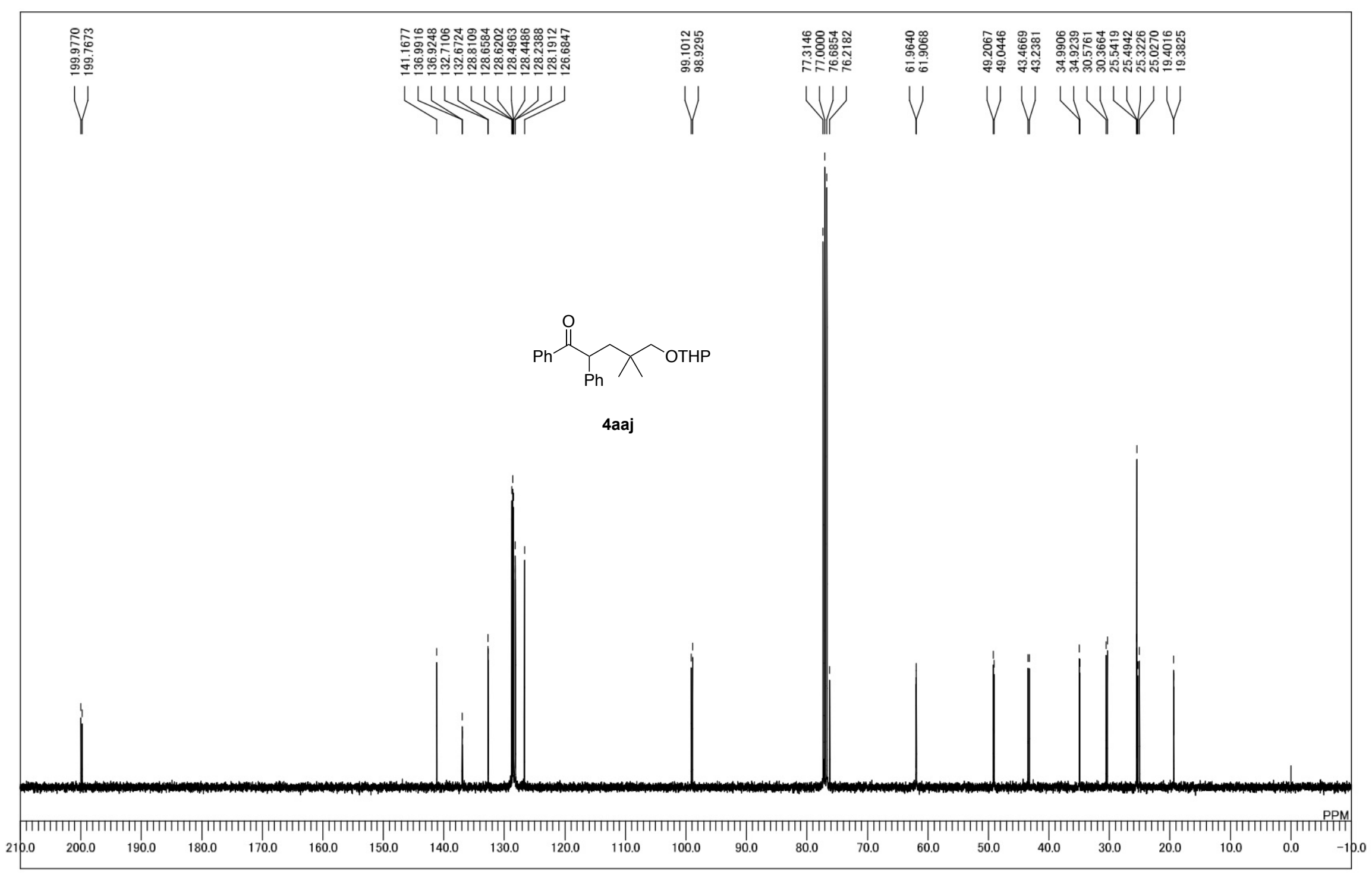

${ }^{13} \mathrm{C}$ NMR spectrum of 4aaj 


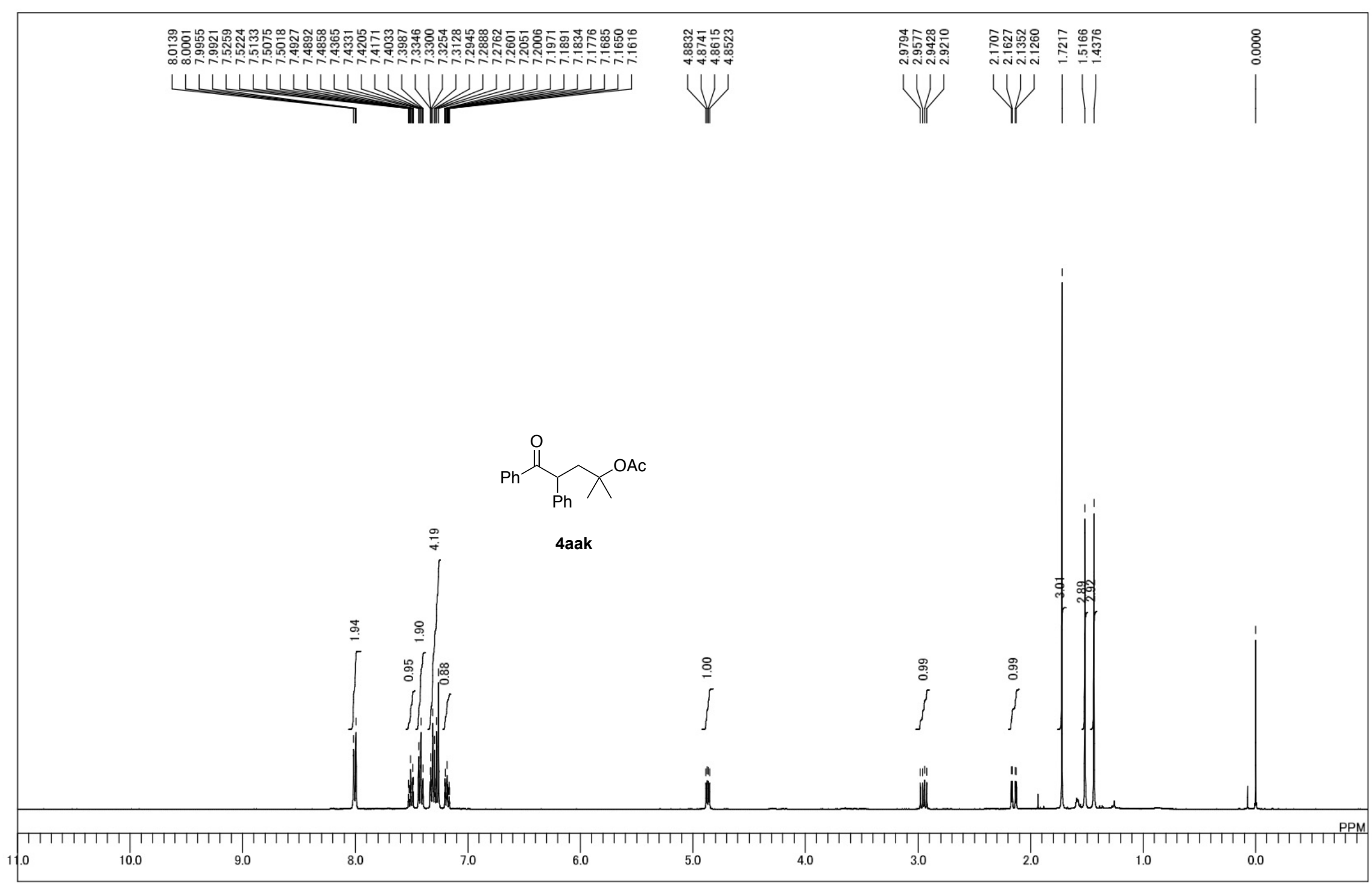

${ }^{1} \mathrm{H}$ NMR spectrum of $\mathbf{4 a a k}$ 


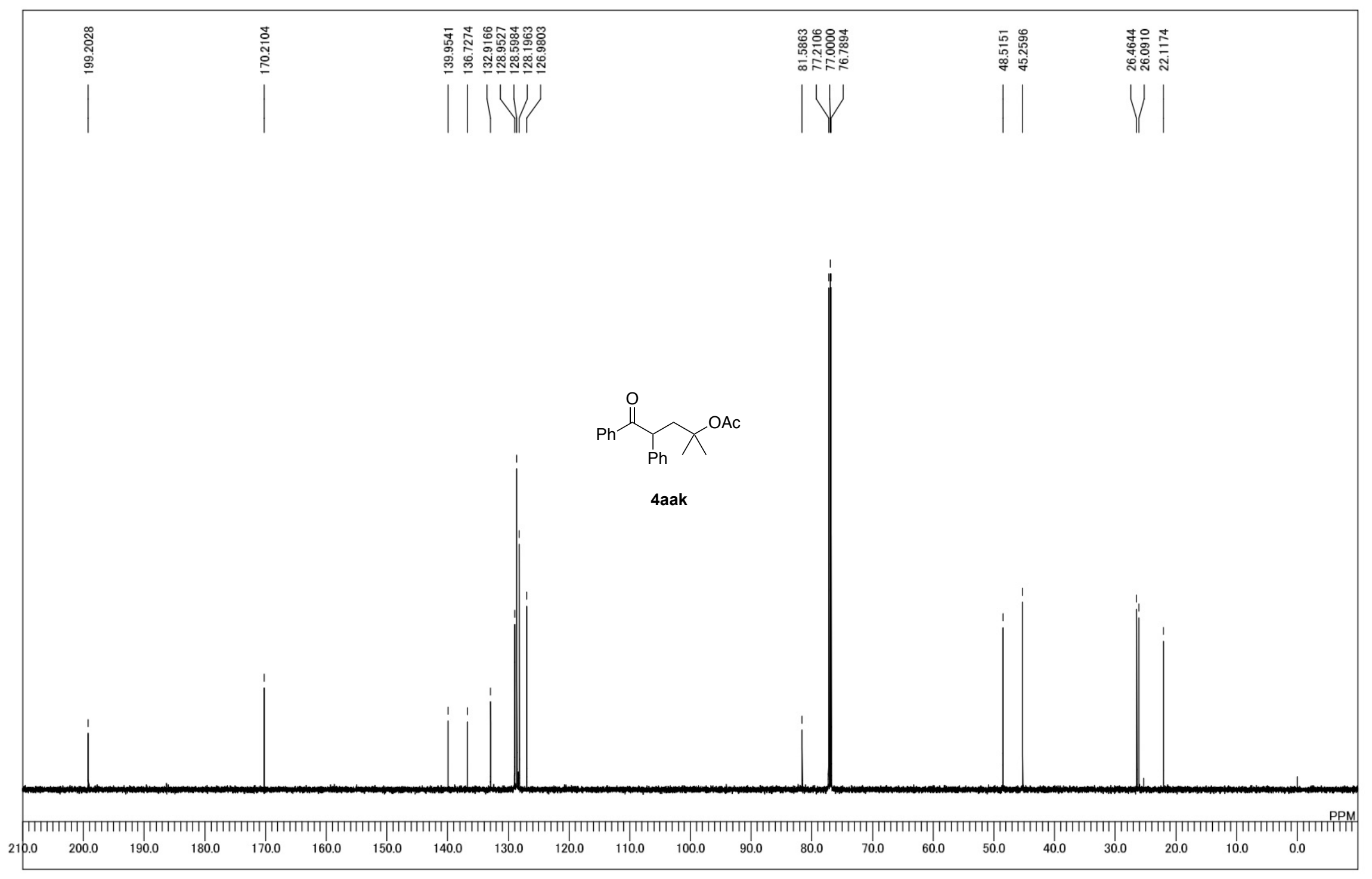

${ }^{13} \mathrm{C}$ NMR spectrum of $\mathbf{4 a a k}$ 


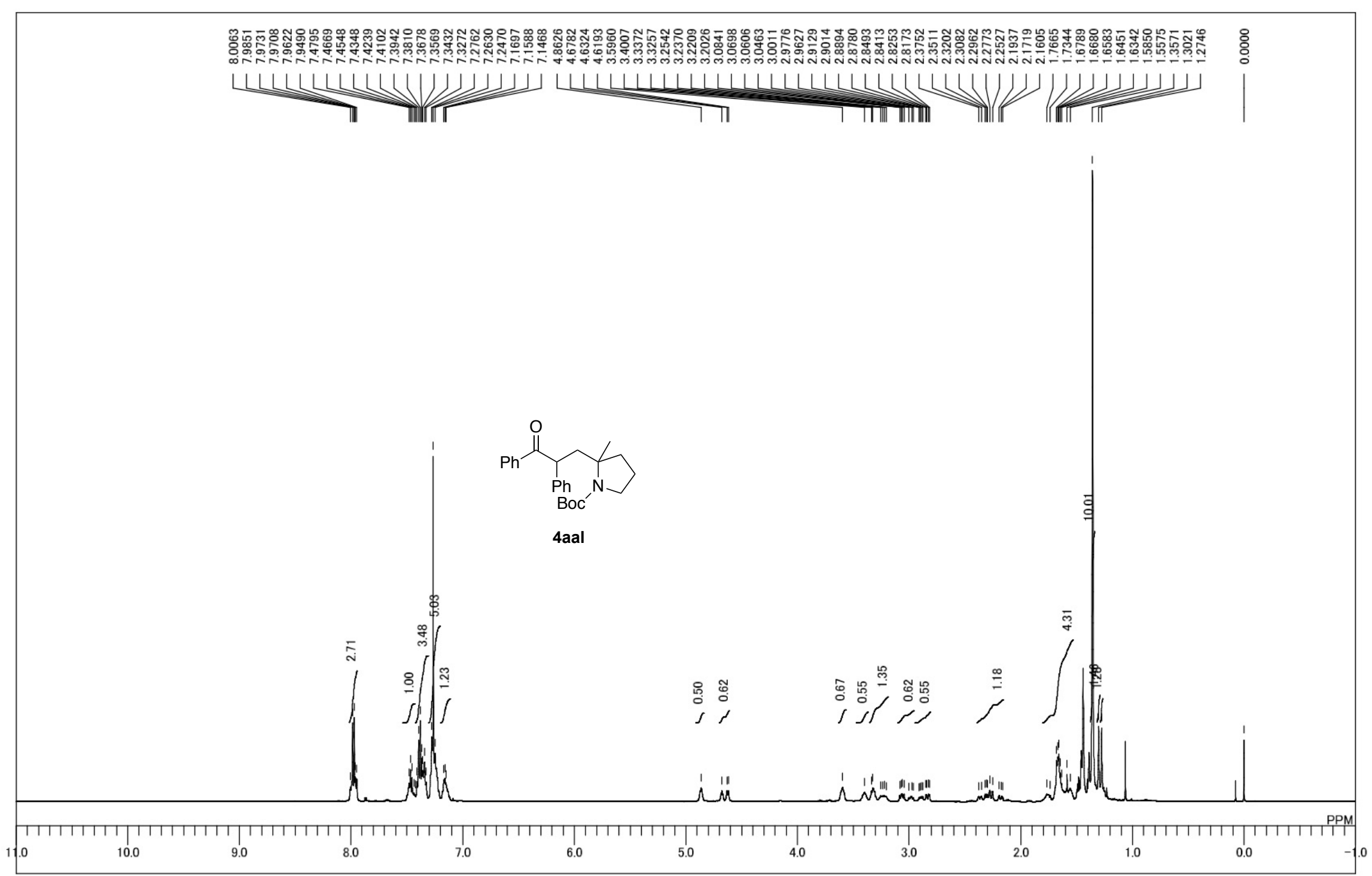

${ }^{1} \mathrm{H}$ NMR spectrum of 4aal 


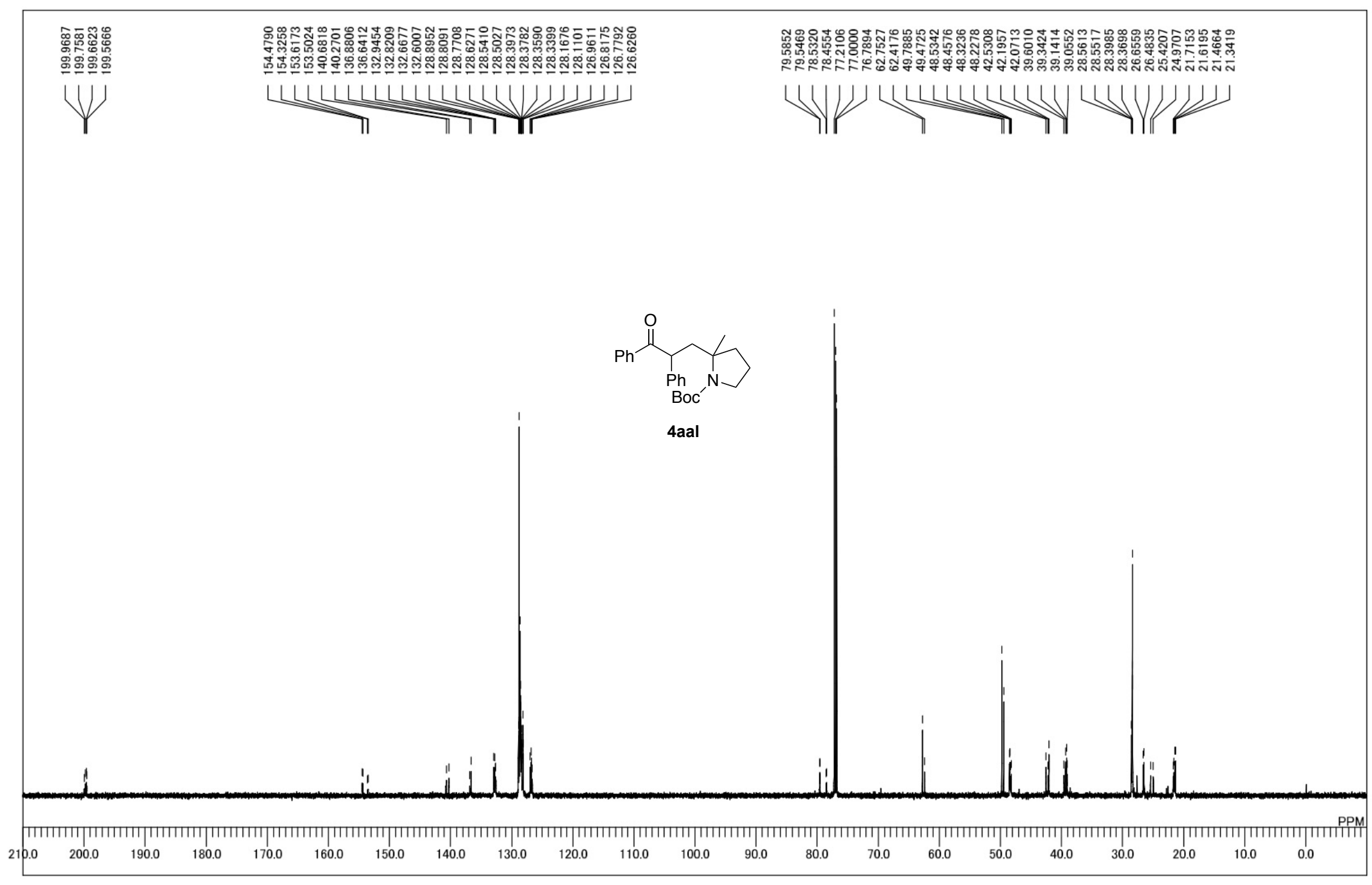

${ }^{13} \mathrm{C}$ NMR spectrum of 4 aal 


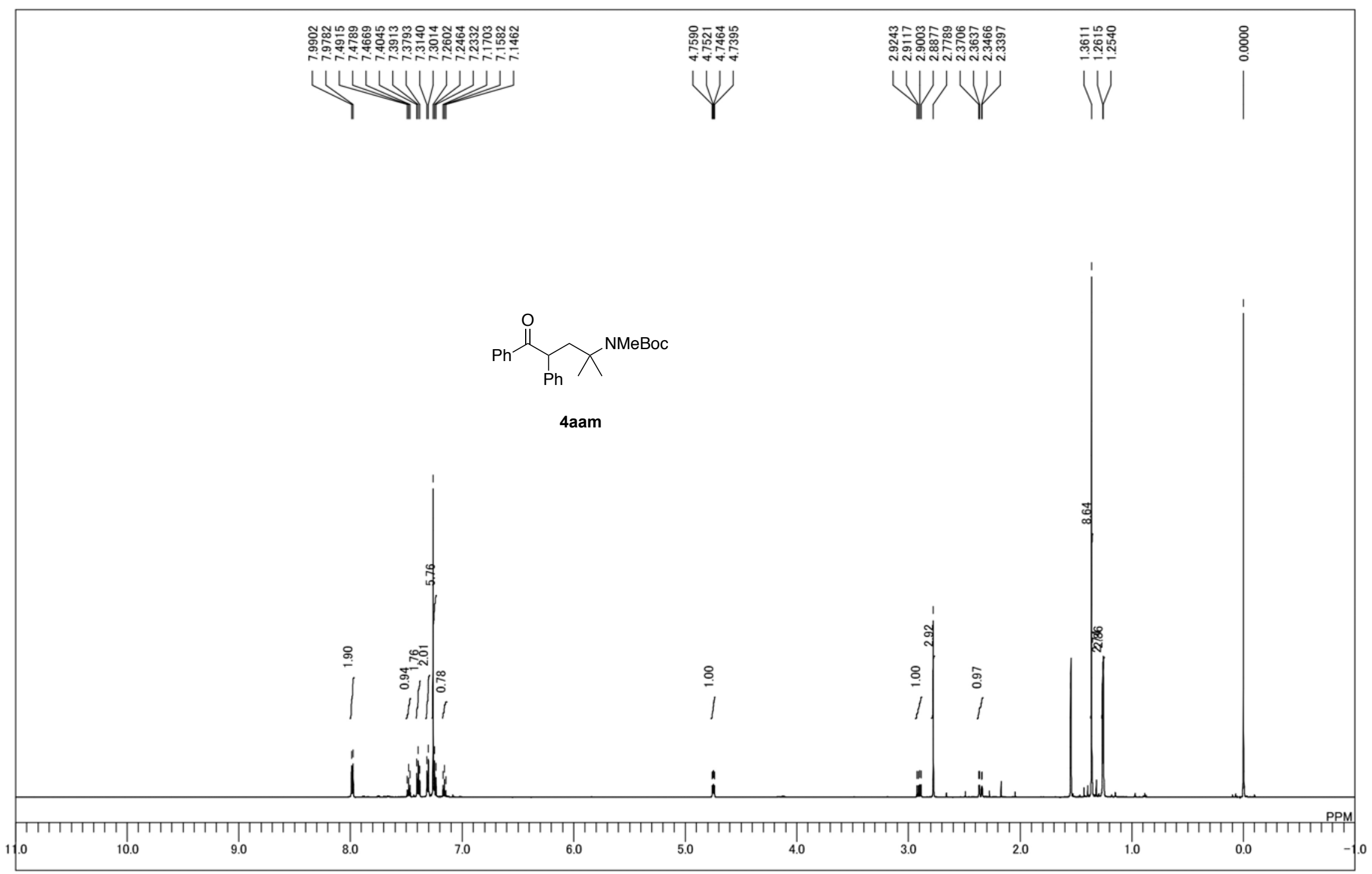

${ }^{1} \mathrm{H}$ NMR spectrum of $\mathbf{4 a a m}$ 


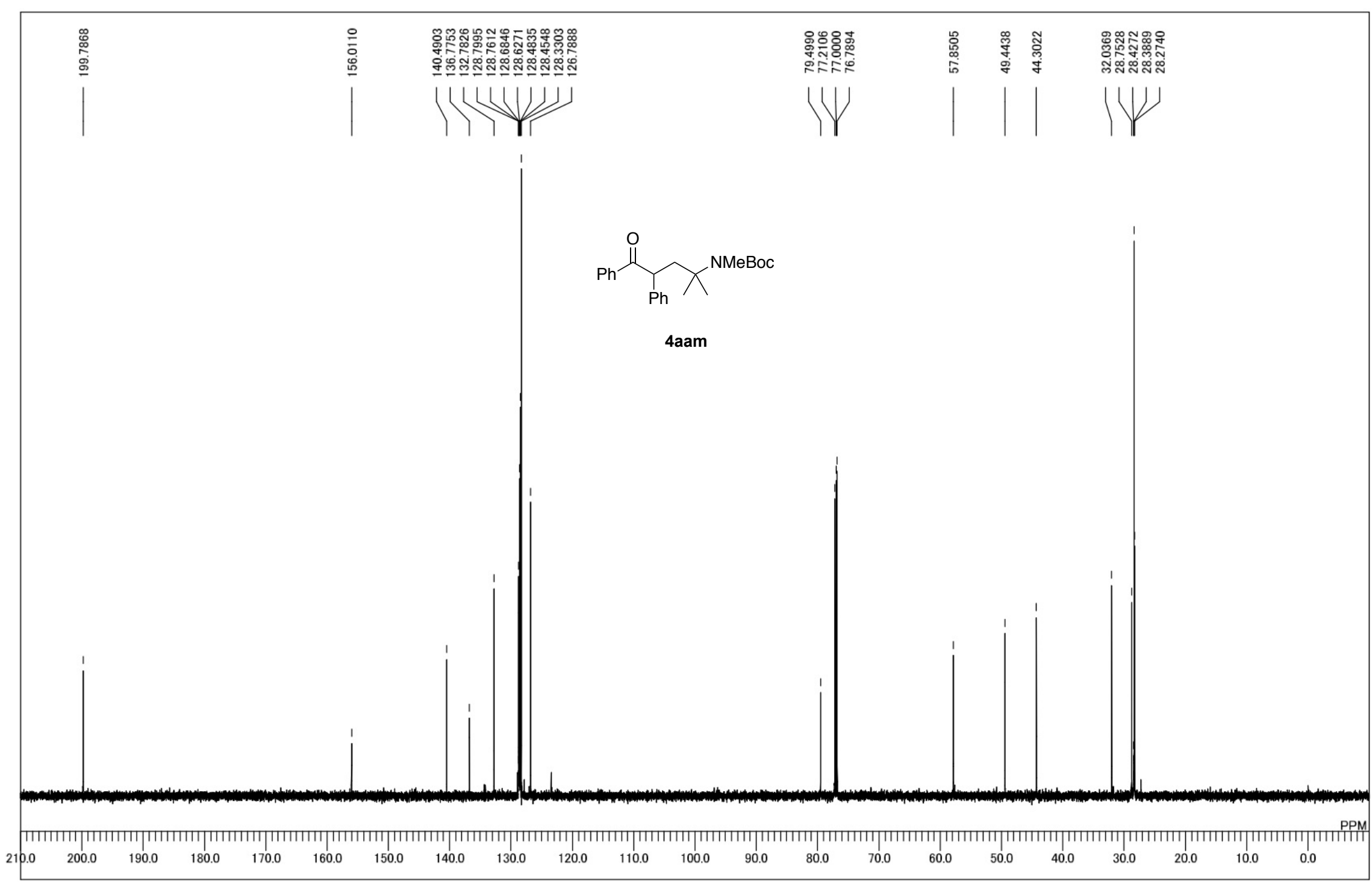

${ }^{13} \mathrm{C}$ NMR spectrum of 4aam 


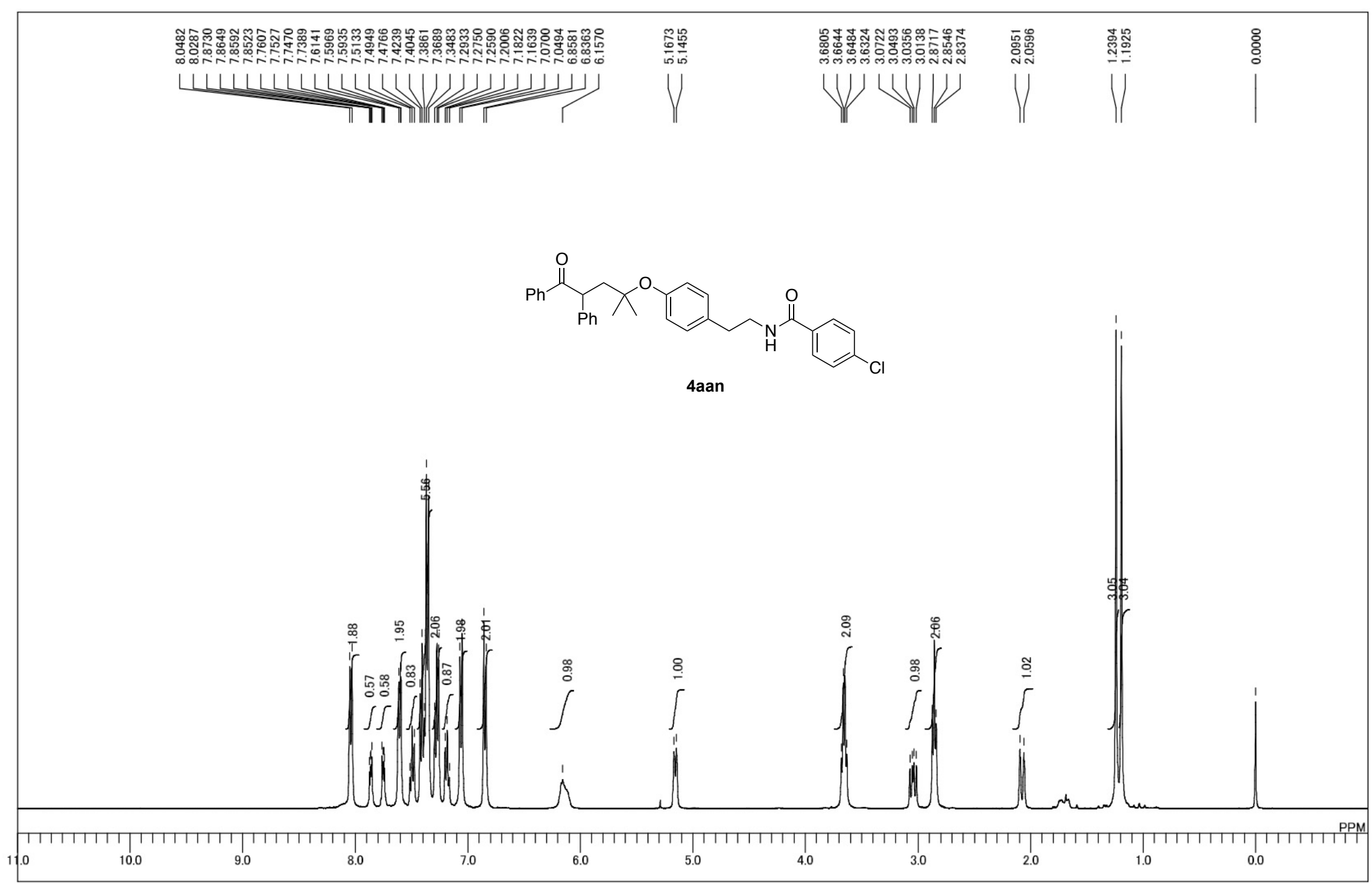

${ }^{1} \mathrm{H}$ NMR spectrum of 4 aan 


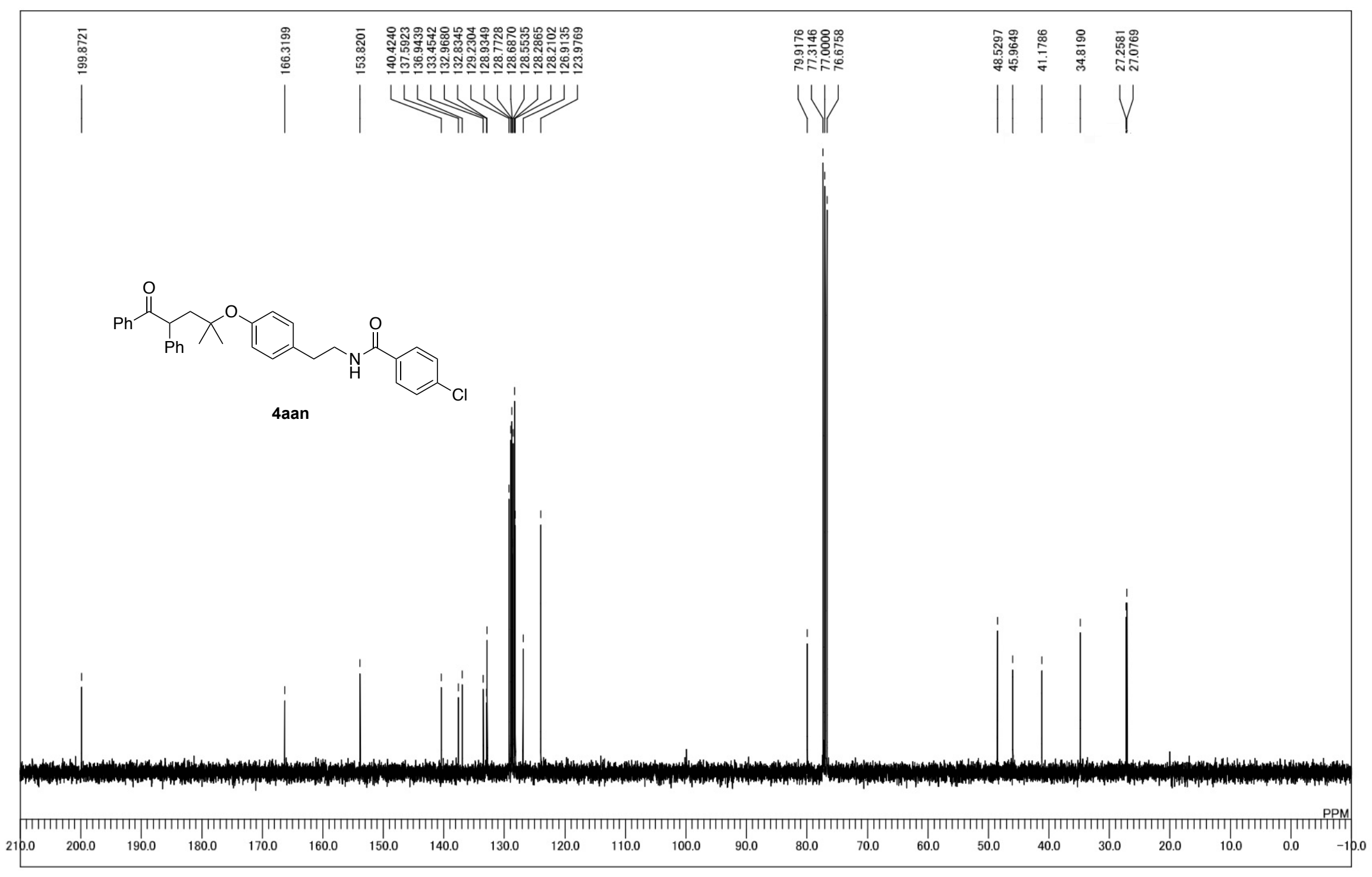

${ }^{13} \mathrm{C}$ NMR spectrum of 4 aan 


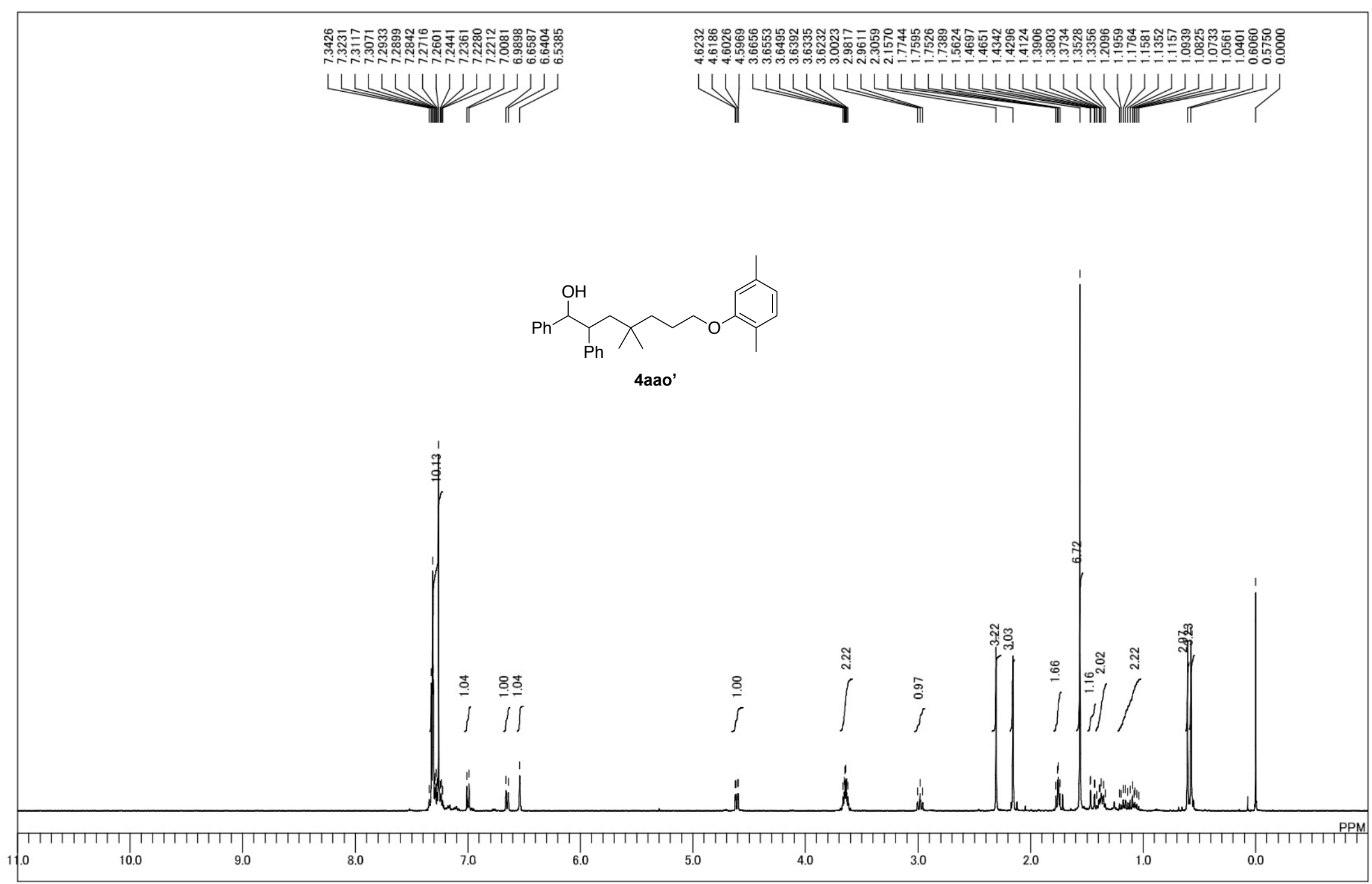

${ }^{1} \mathrm{H}$ NMR spectrum of $\mathbf{4 a a o}$ 


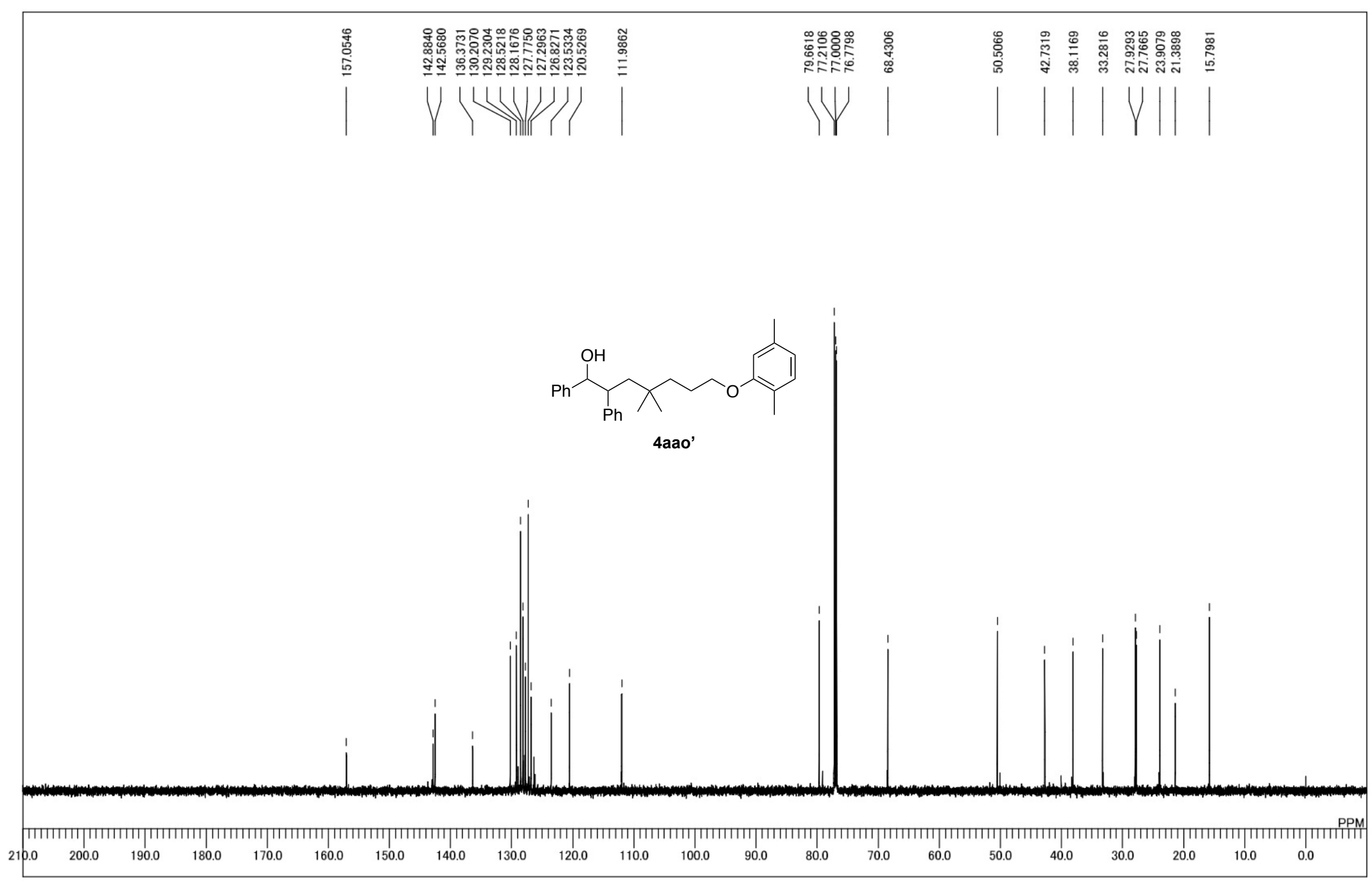

${ }^{13} \mathrm{C}$ NMR spectrum of 4aao' 


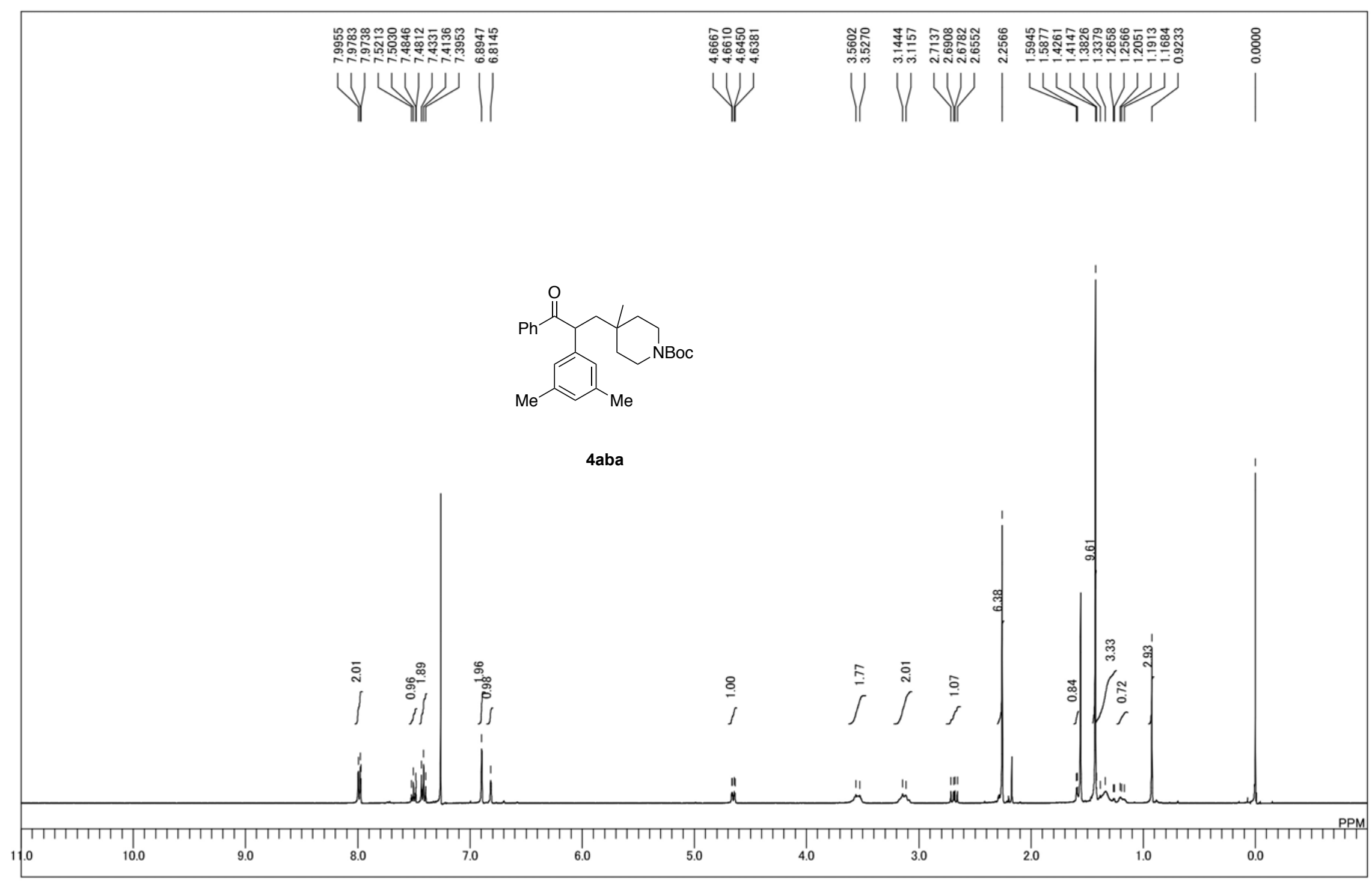

${ }^{1} \mathrm{H}$ NMR spectrum of $\mathbf{4 a b a}$ 


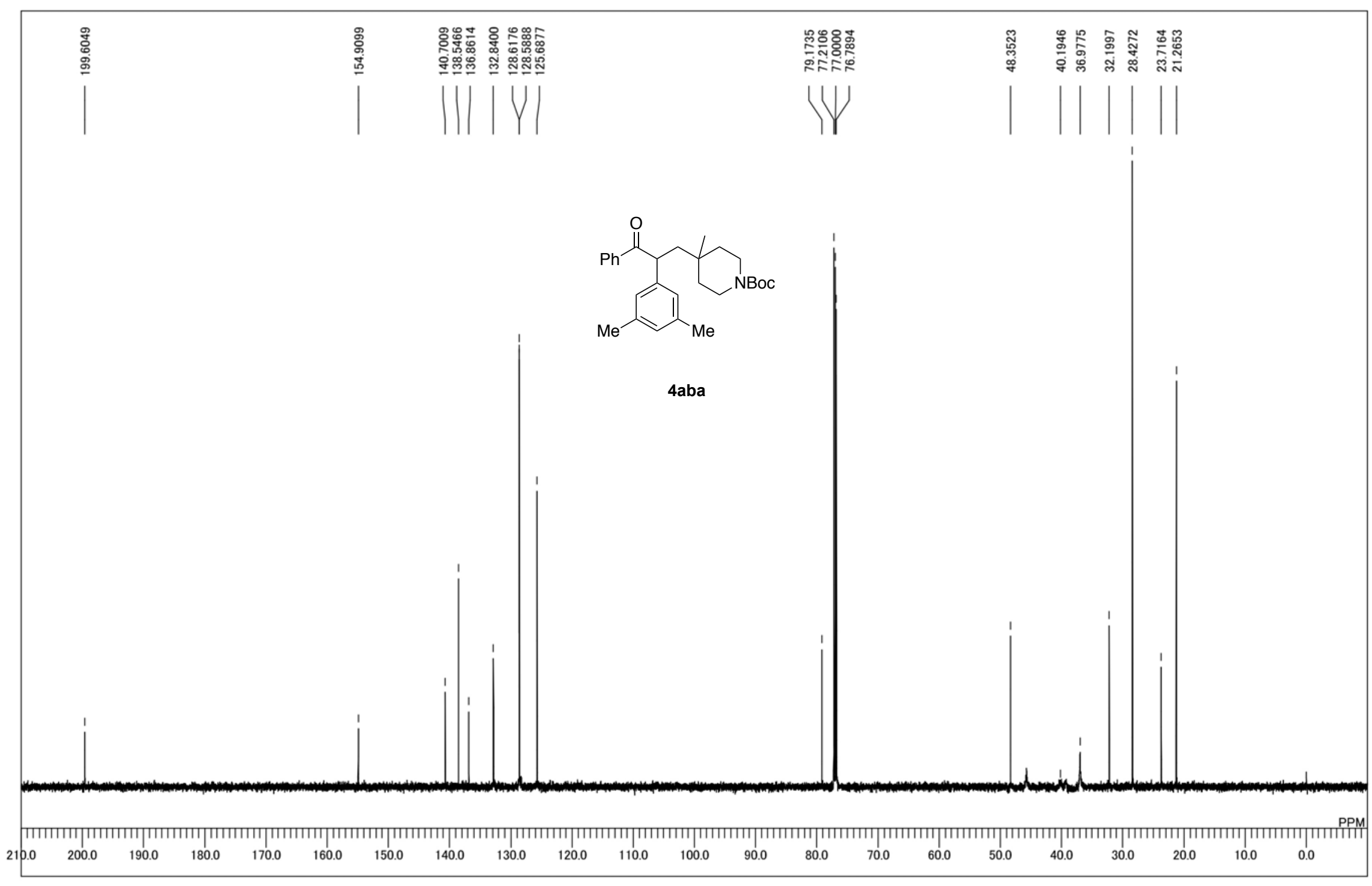

${ }^{13} \mathrm{C}$ NMR spectrum of 4 aba 


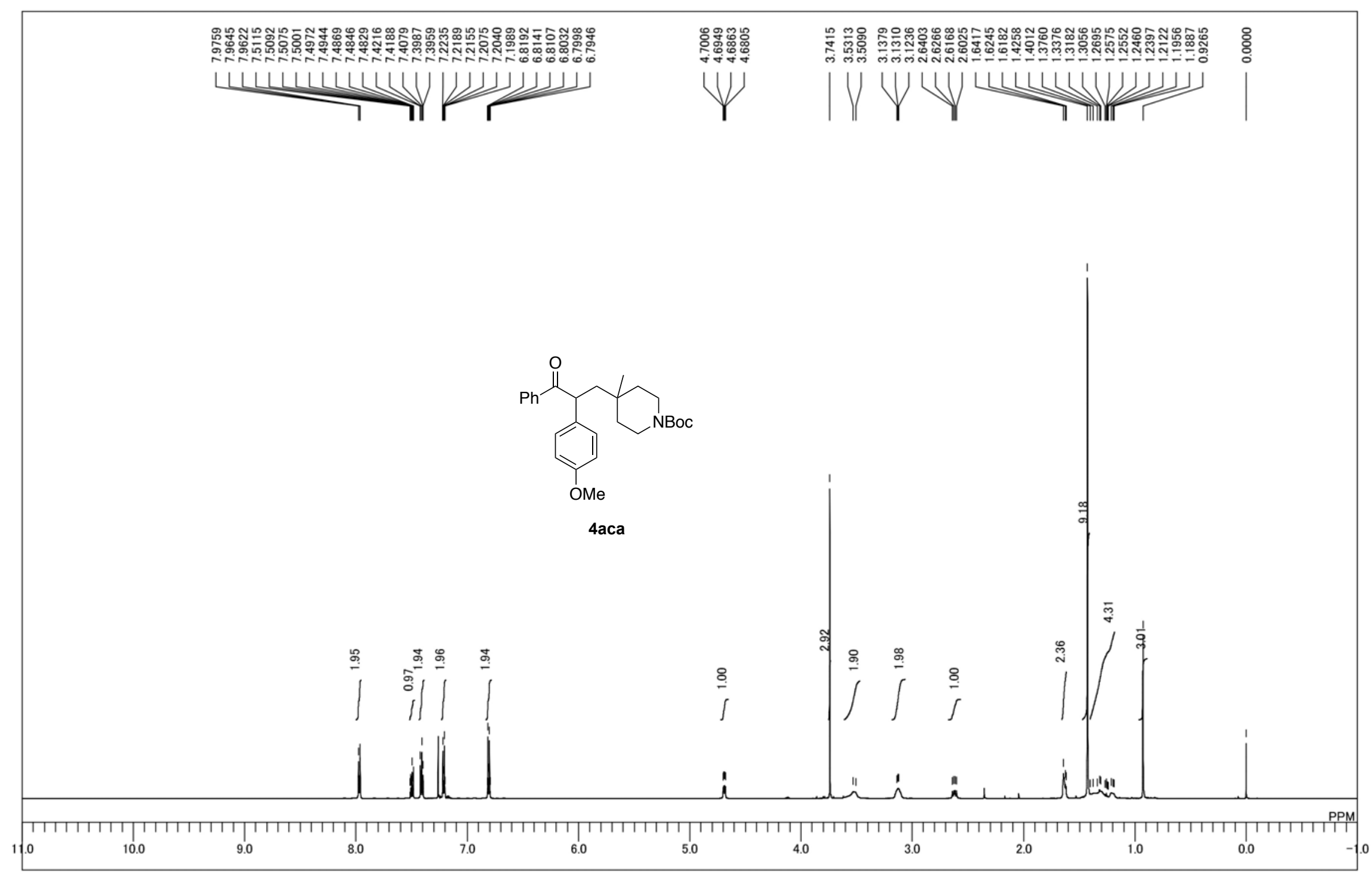

${ }^{1} \mathrm{H}$ NMR spectrum of 4aca 


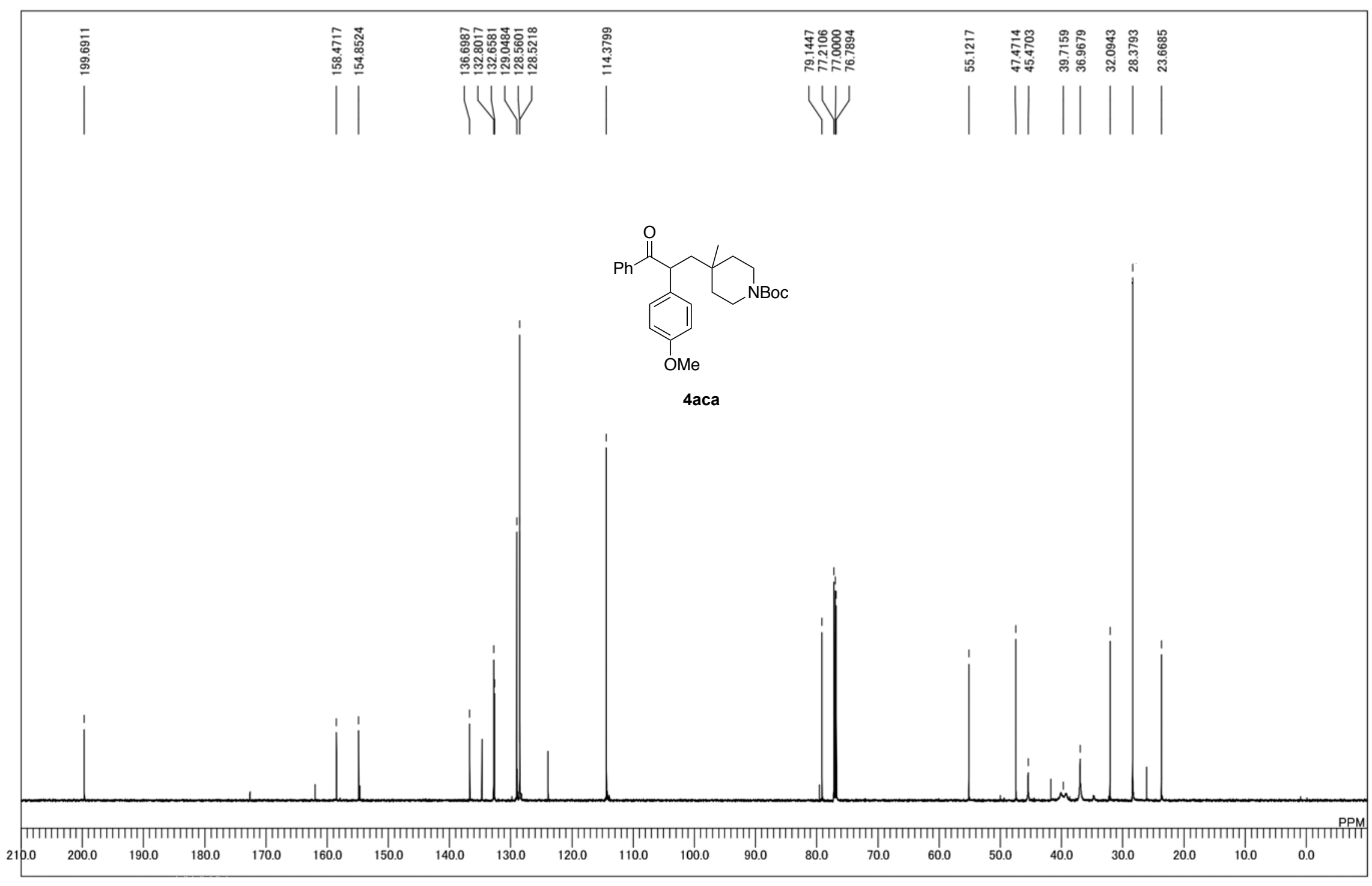

${ }^{13} \mathrm{C}$ NMR spectrum of 4 aca 


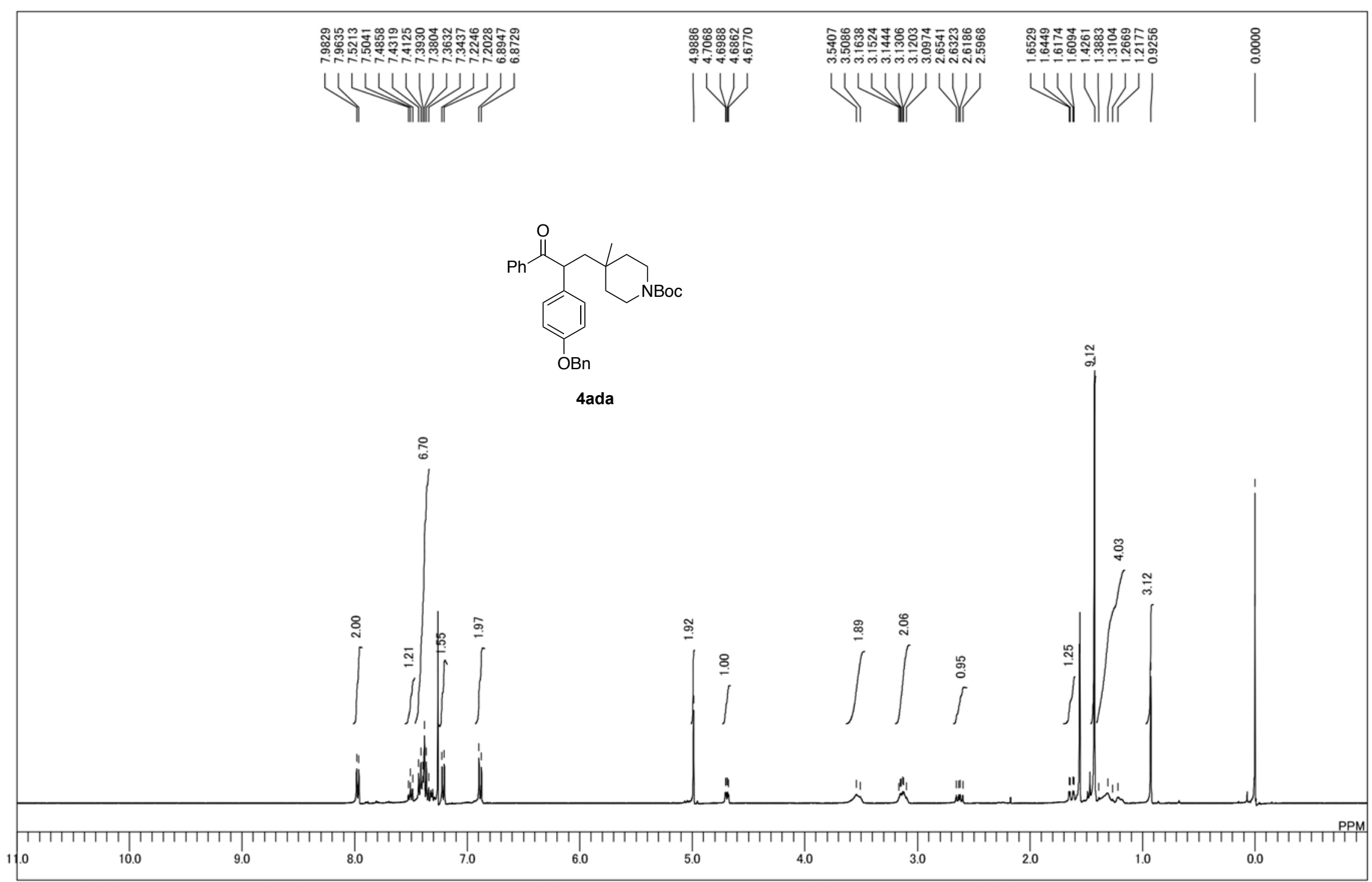

${ }^{1} \mathrm{H}$ NMR spectrum of $\mathbf{4 a d a}$ 


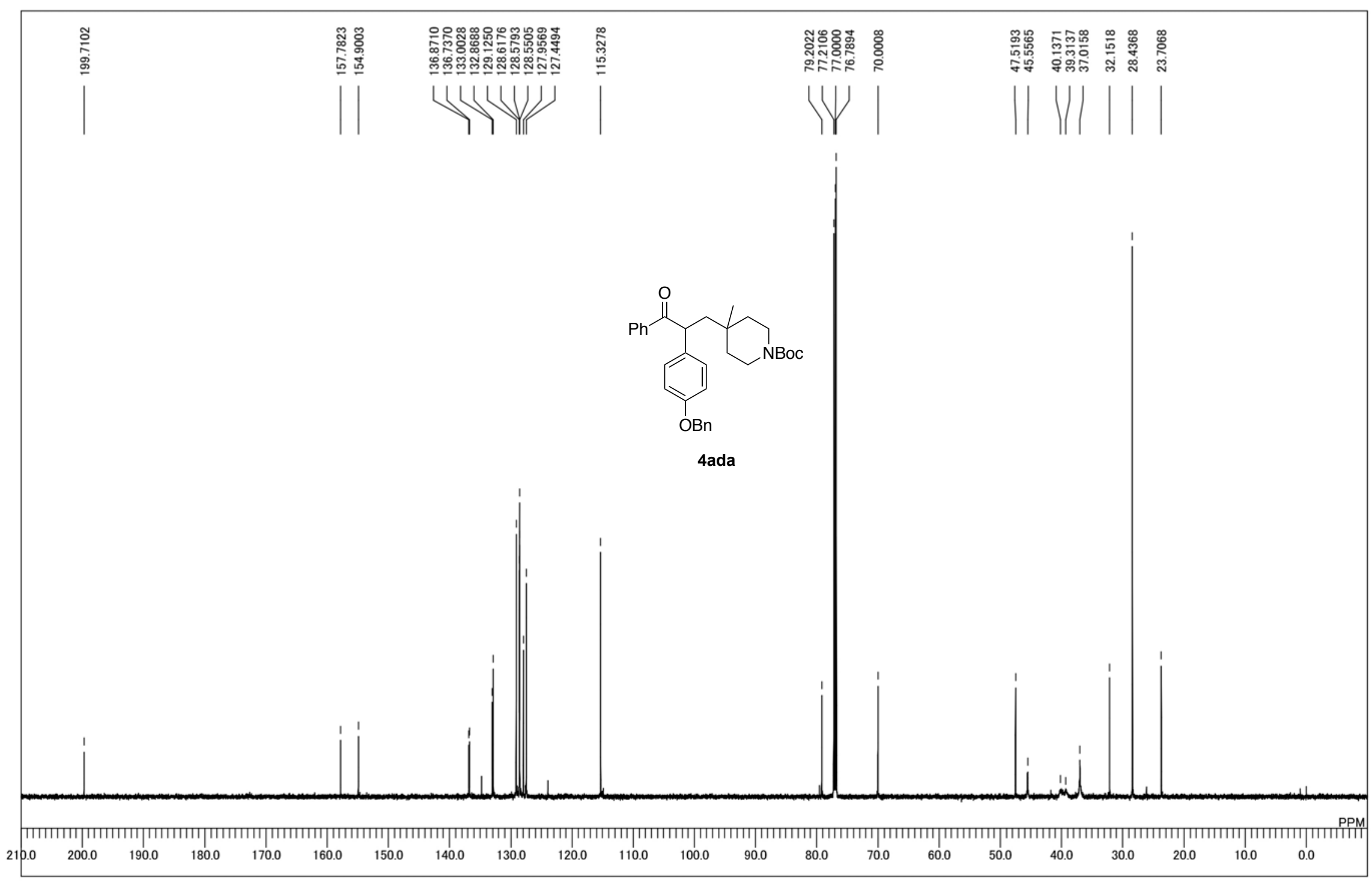

${ }^{13} \mathrm{C}$ NMR spectrum of 4 ada 


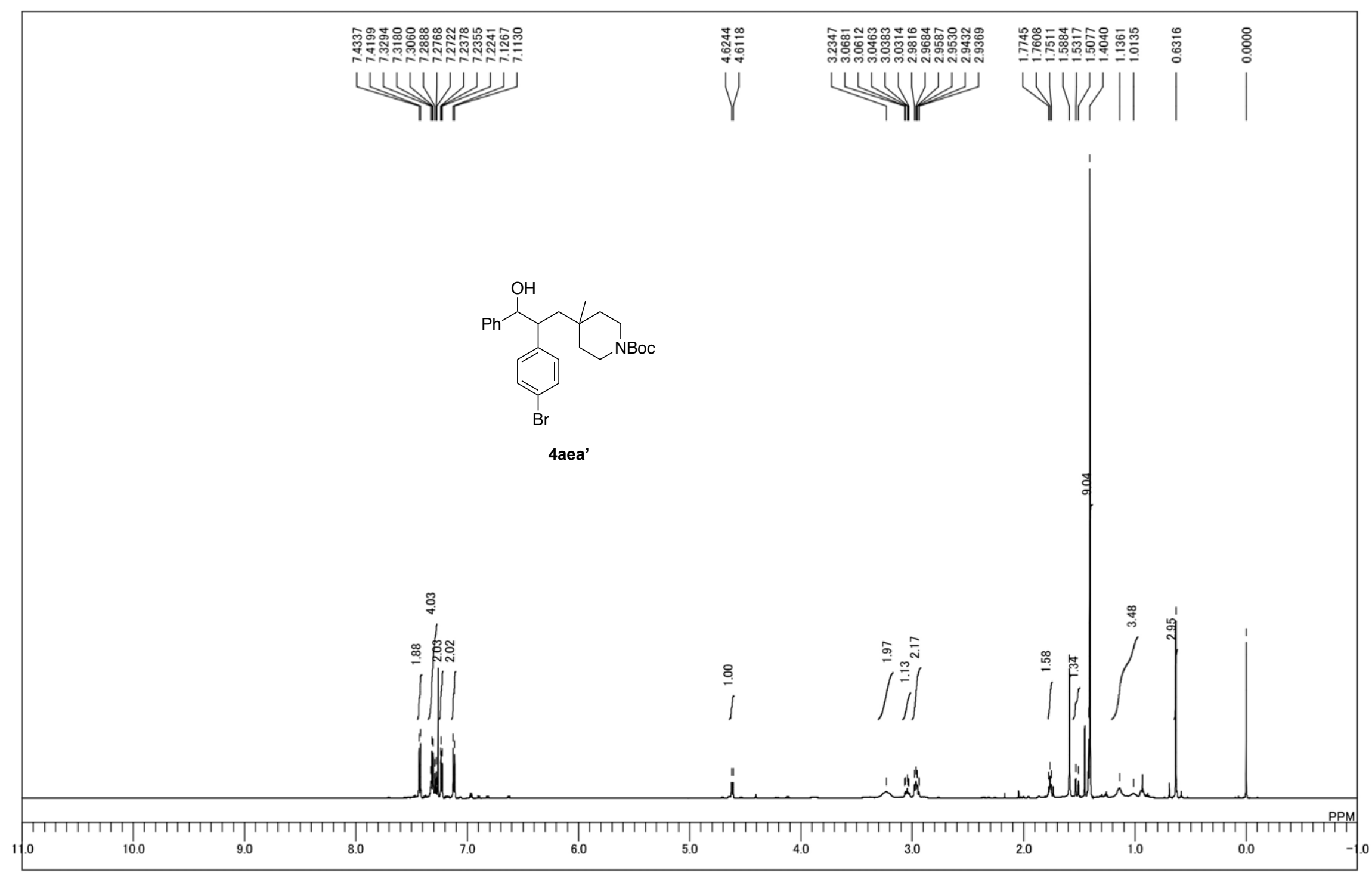

${ }^{1} \mathrm{H}$ NMR spectrum of $\mathbf{4 a e a}$ 


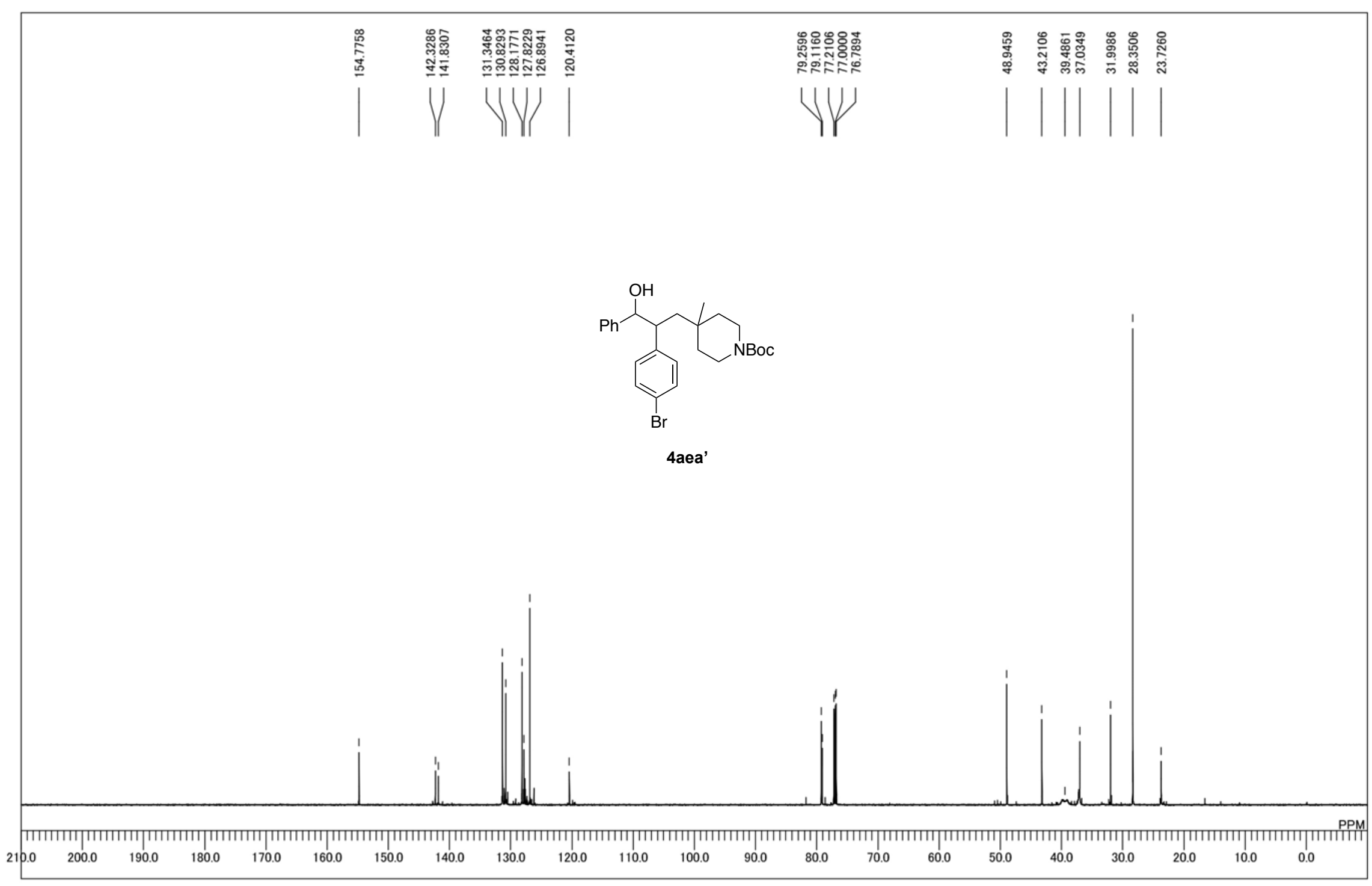

${ }^{13} \mathrm{C}$ NMR spectrum of $\mathbf{4 a e a}$ 


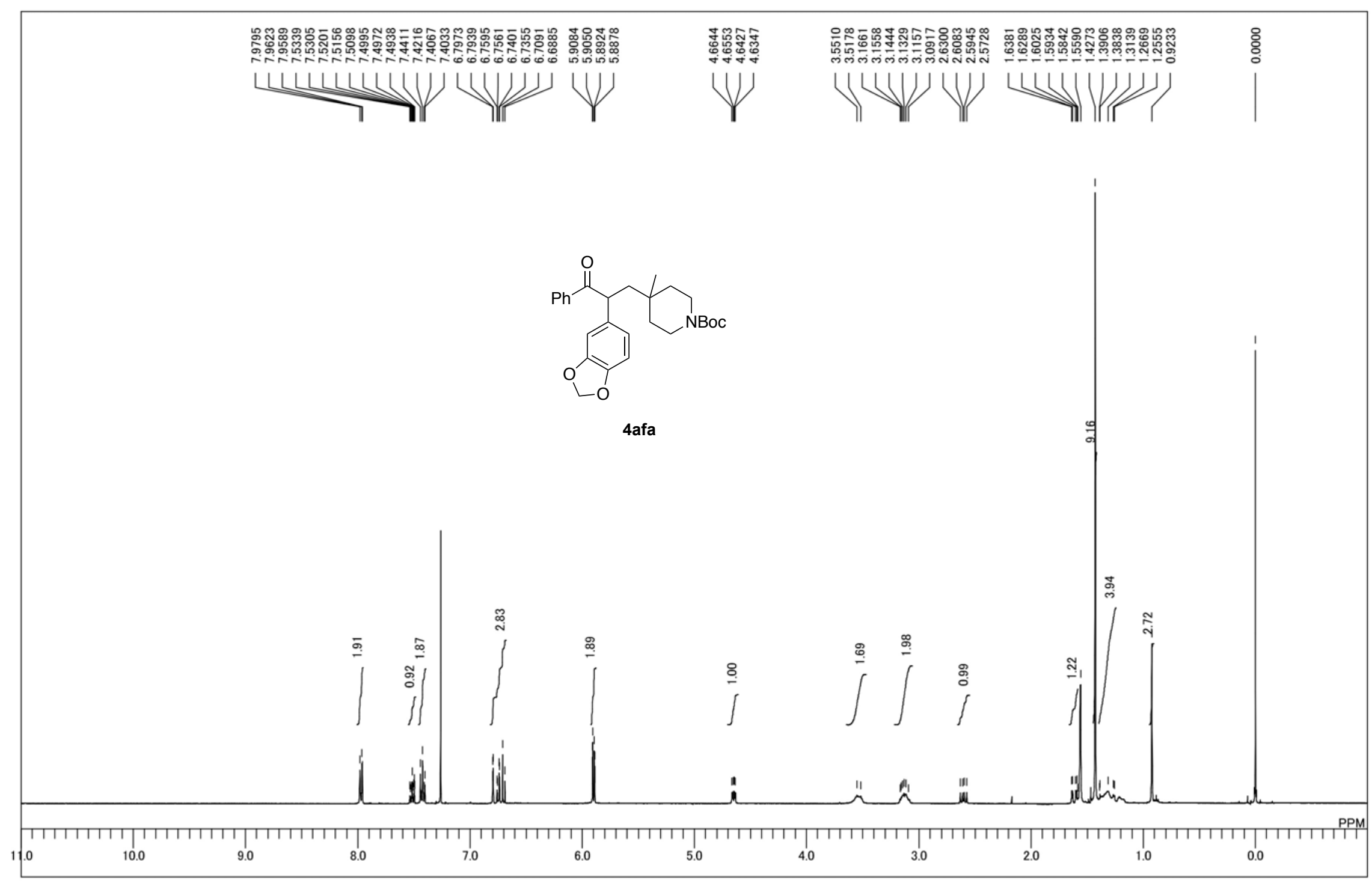

${ }^{1} \mathrm{H}$ NMR spectrum of $\mathbf{4 a f a}$ 


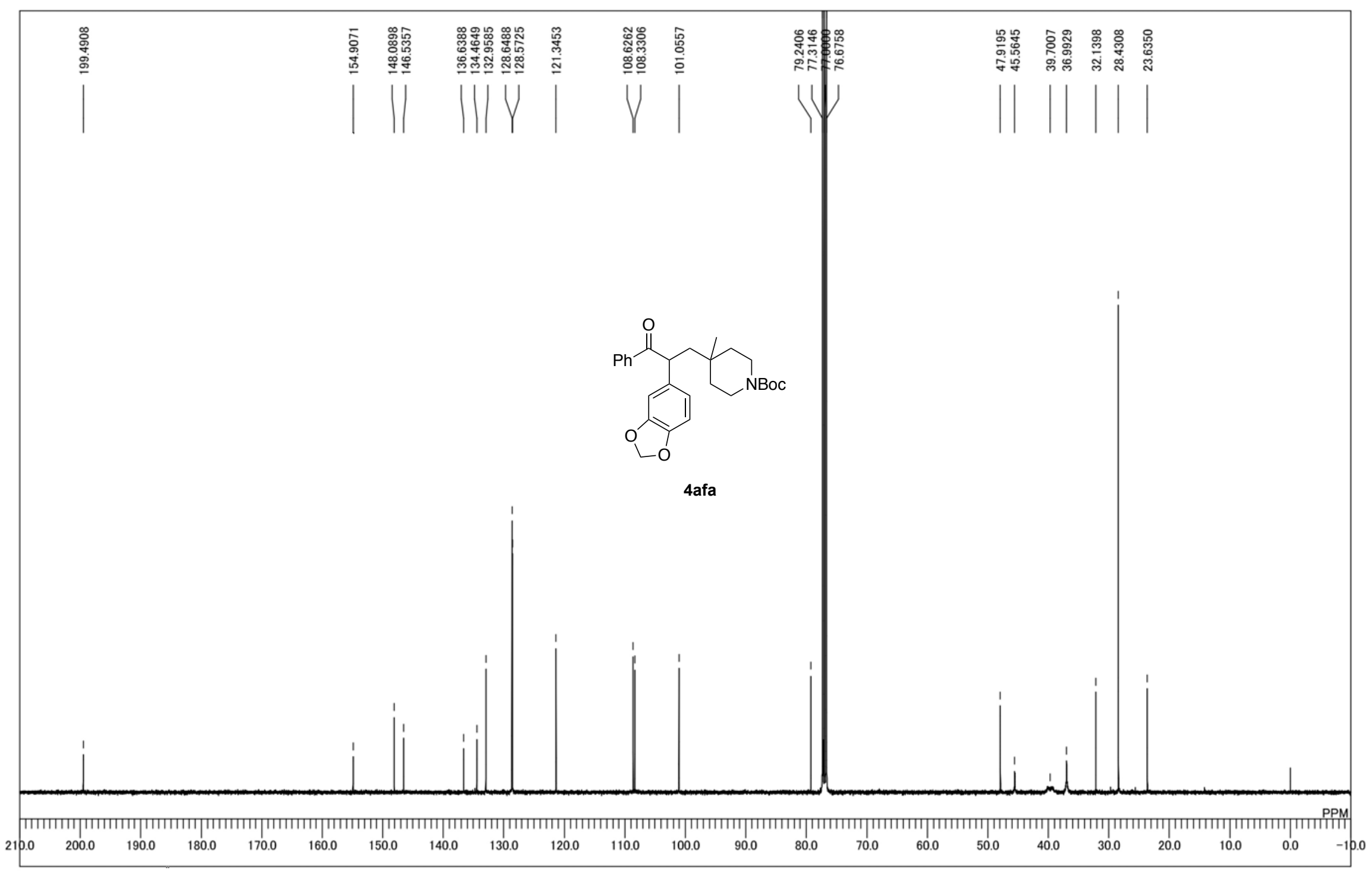

${ }^{13} \mathrm{C}$ NMR spectrum of $\mathbf{4 a f a}$ 


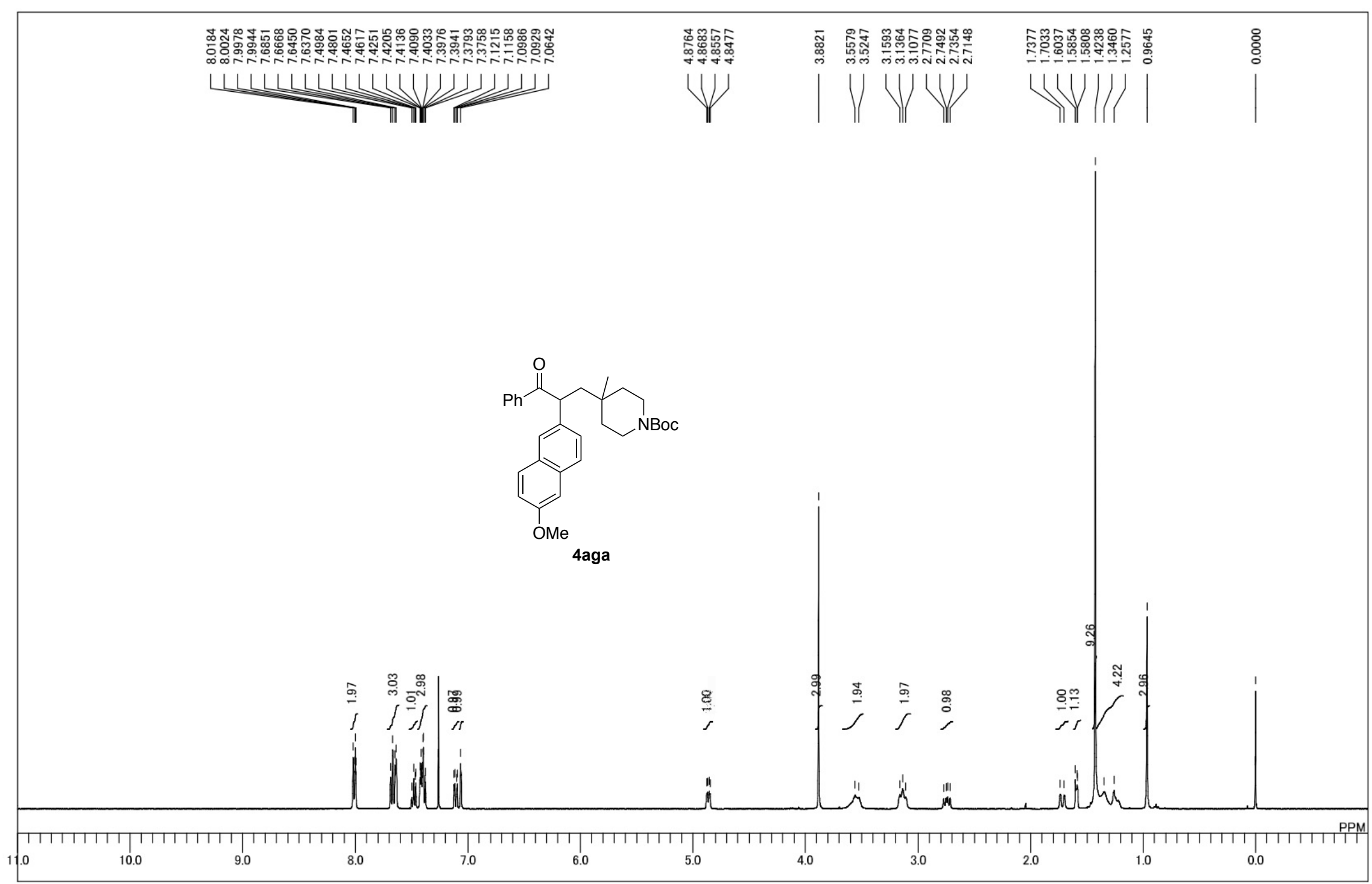

${ }^{1} \mathrm{H}$ NMR spectrum of 4 aga 


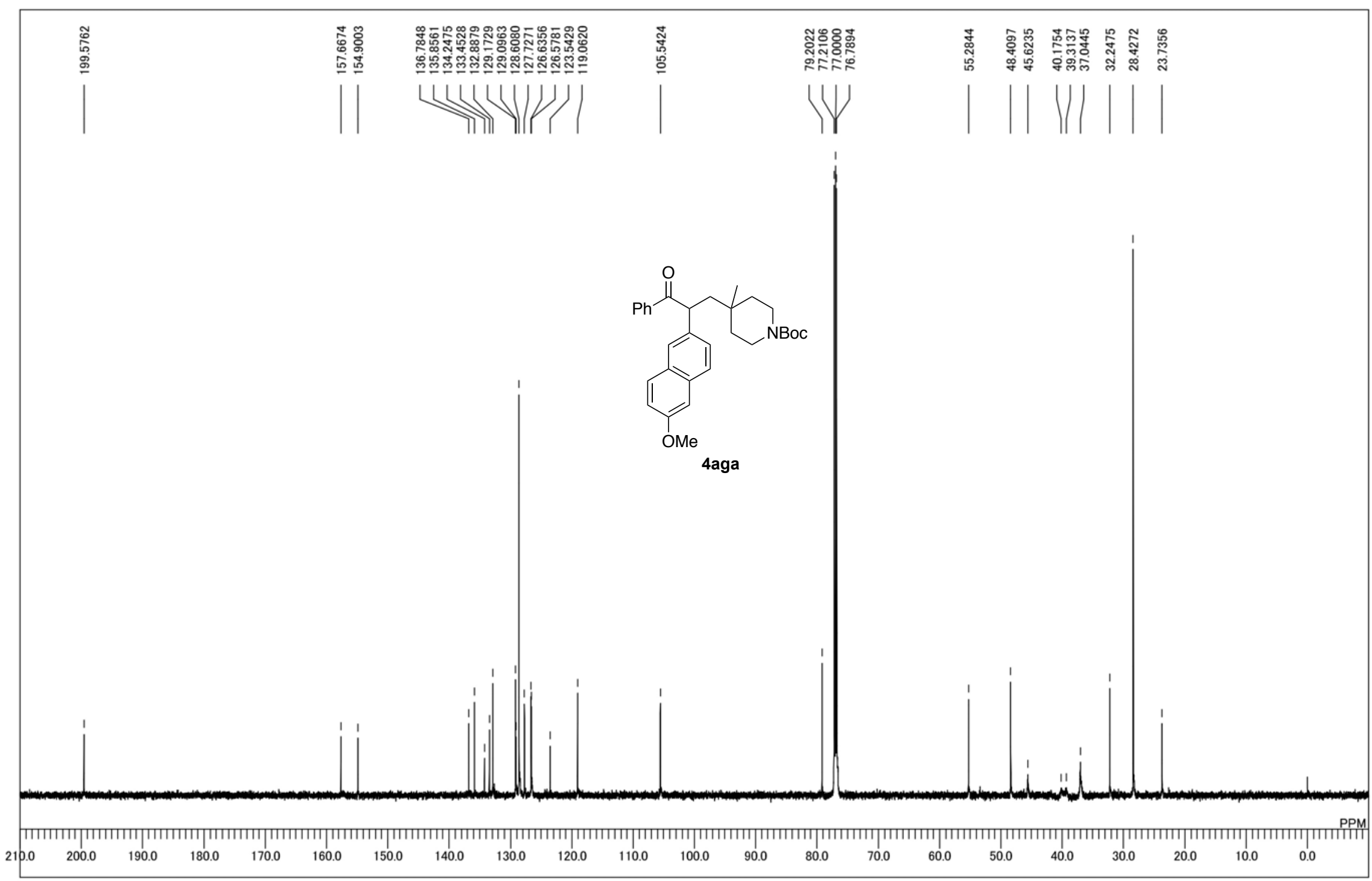

${ }^{13} \mathrm{C}$ NMR spectrum of 4 aga 


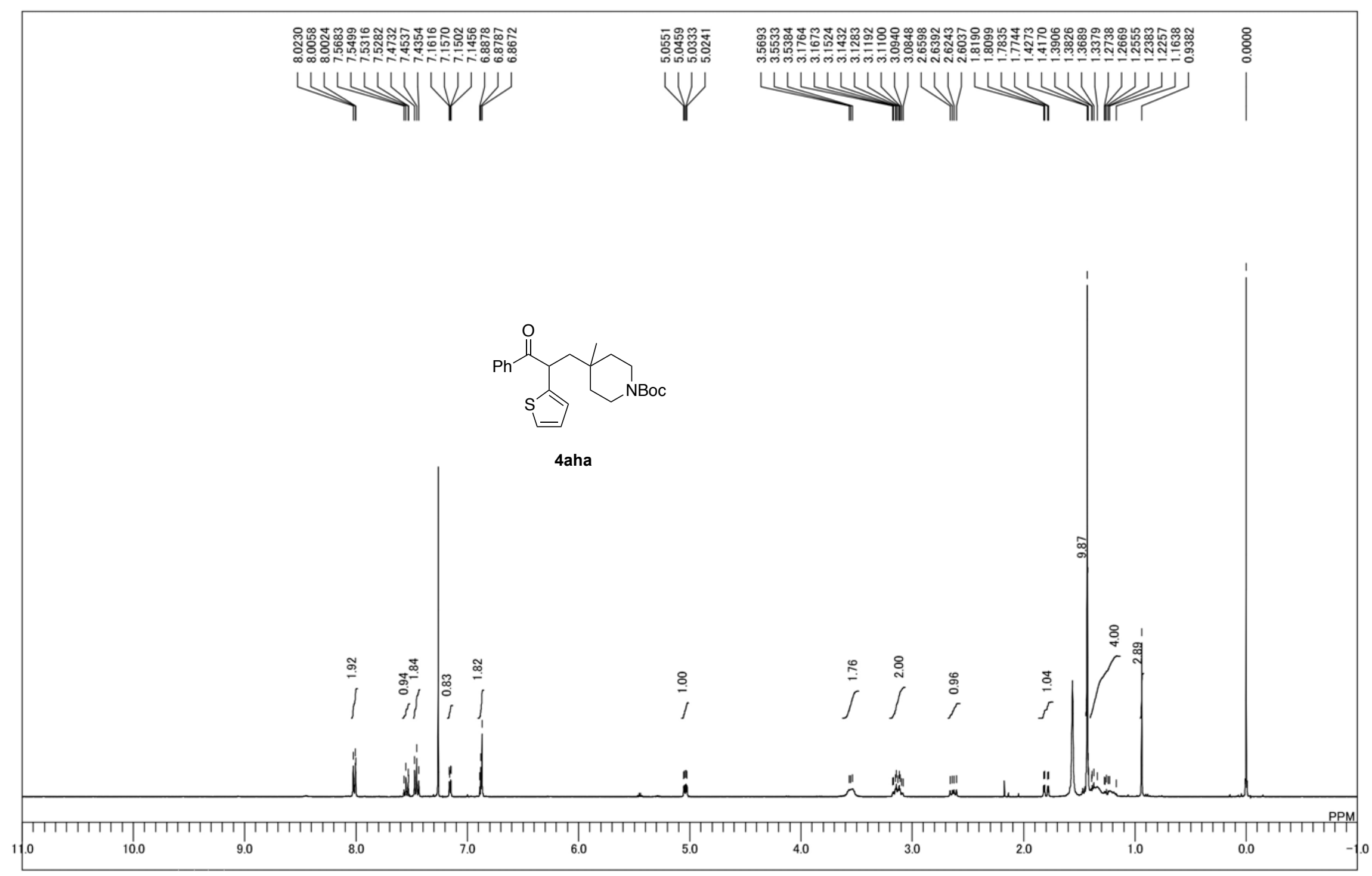

${ }^{1} \mathrm{H}$ NMR spectrum of $\mathbf{4 a h a}$ 


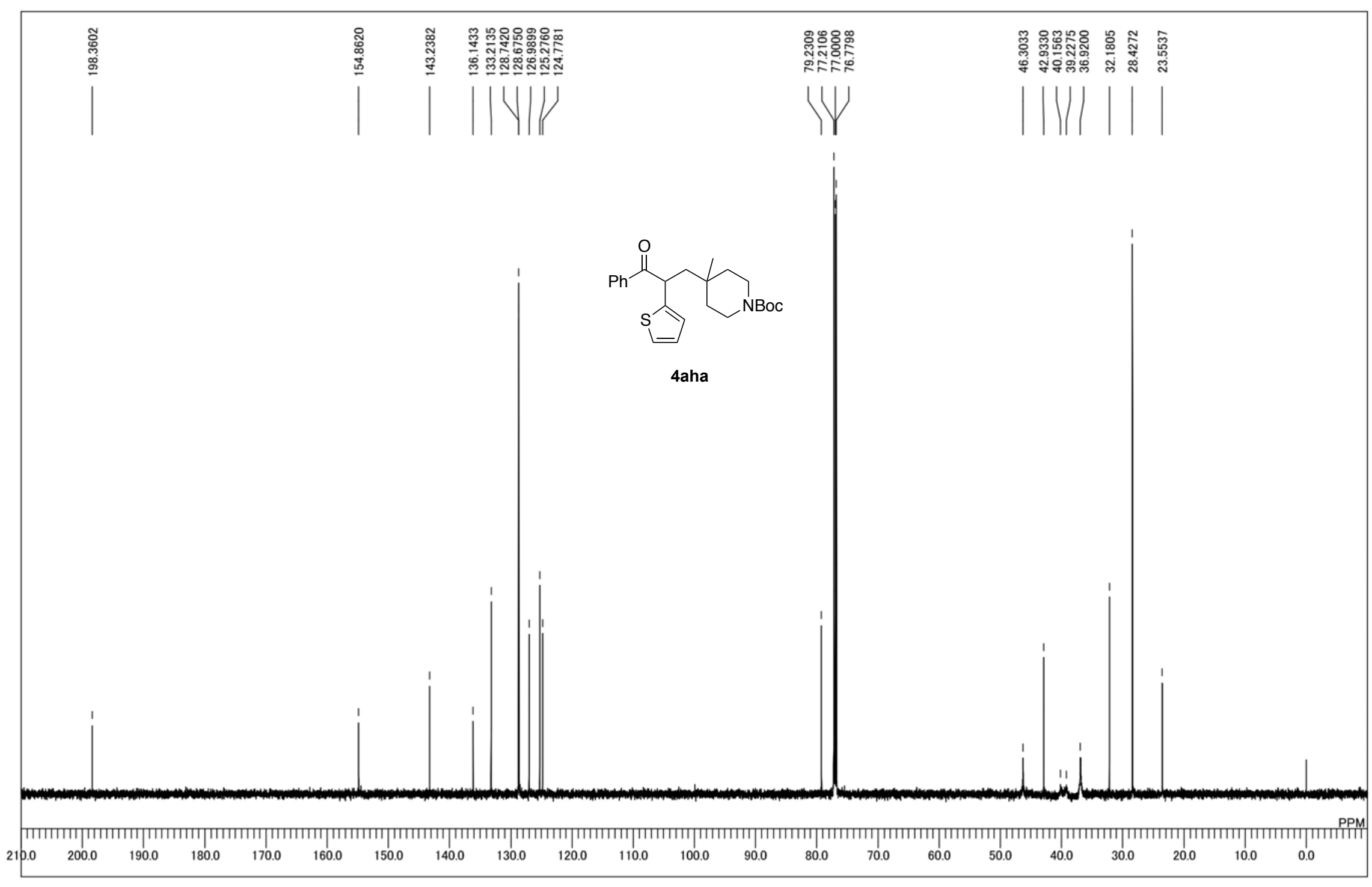

${ }^{13} \mathrm{C}$ NMR spectrum of 4aha 


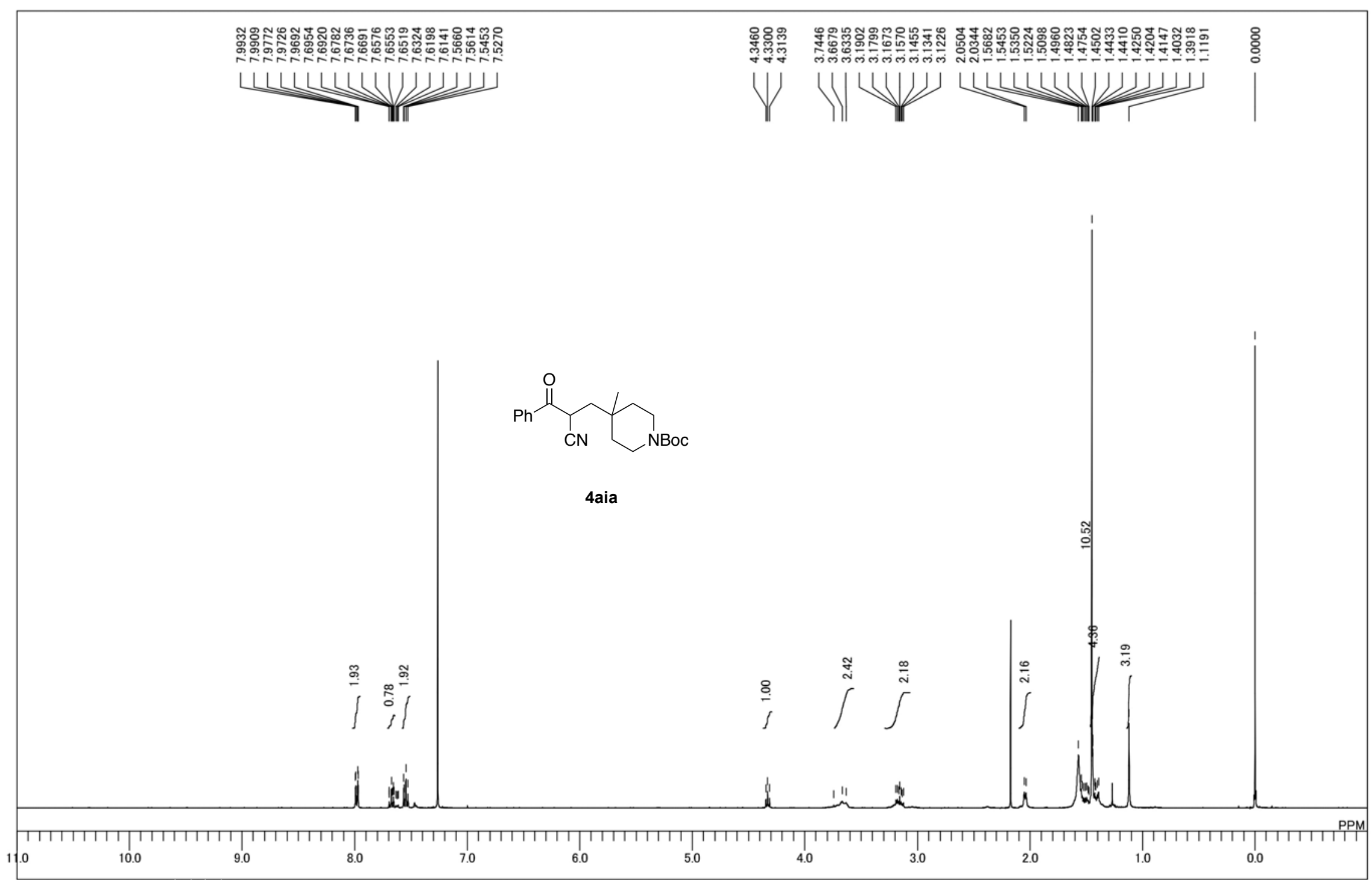

${ }^{1} \mathrm{H}$ NMR spectrum of 4aia 


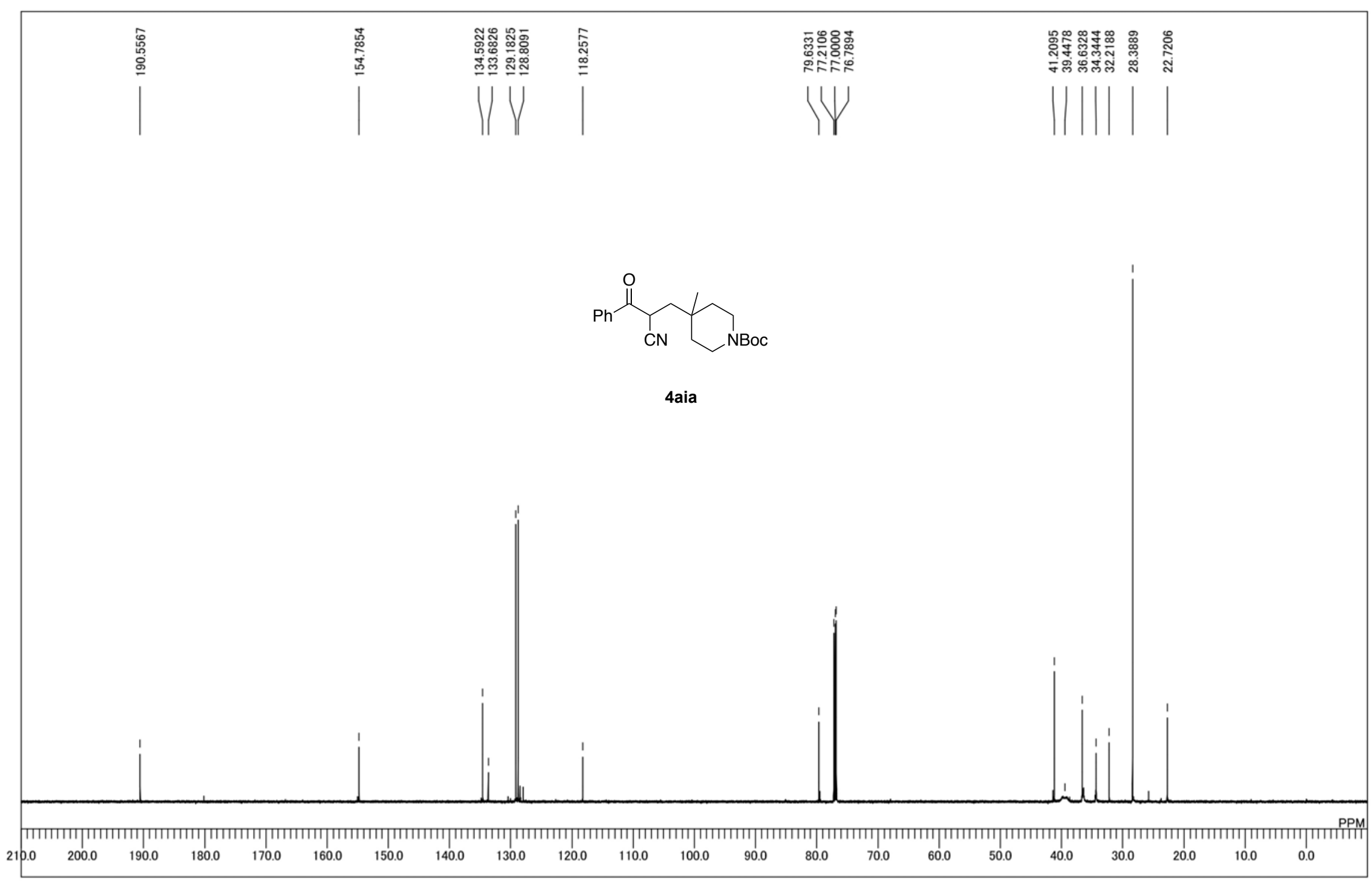

${ }^{13} \mathrm{C}$ NMR spectrum of 4aia 


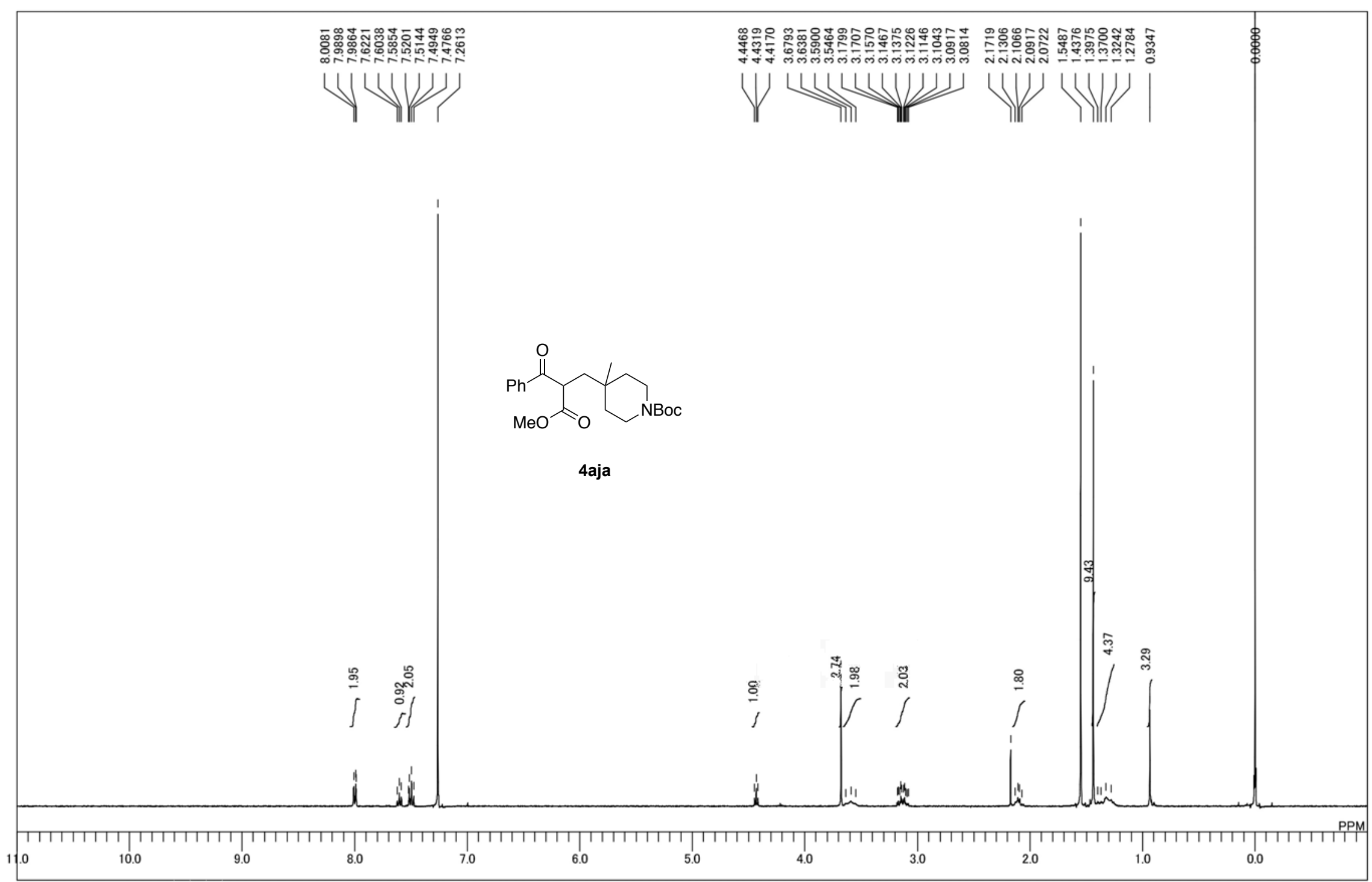

${ }^{1} \mathrm{H}$ NMR spectrum of 4aja 


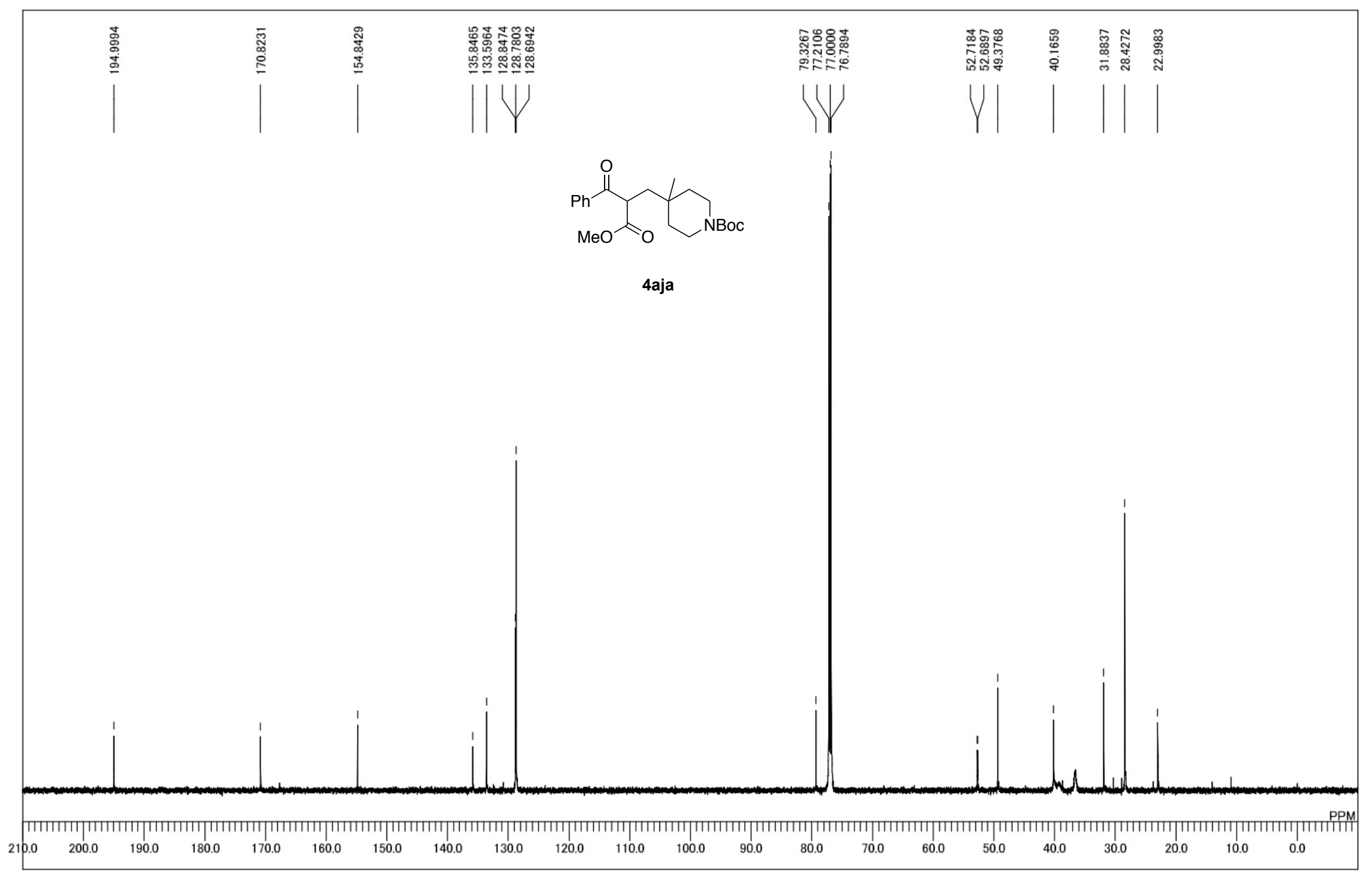

${ }^{13} \mathrm{C}$ NMR spectrum of 4aja 